\title{
NASACR.174, 704
}

\section{A Reproduced Copy}

\author{
OF \\ NASA-CR-174704 \\ 19840023205 \\ - NASACR-194, 704
}

Reproduced for NASA

by the

NASA Scientific and Technical Information Facility

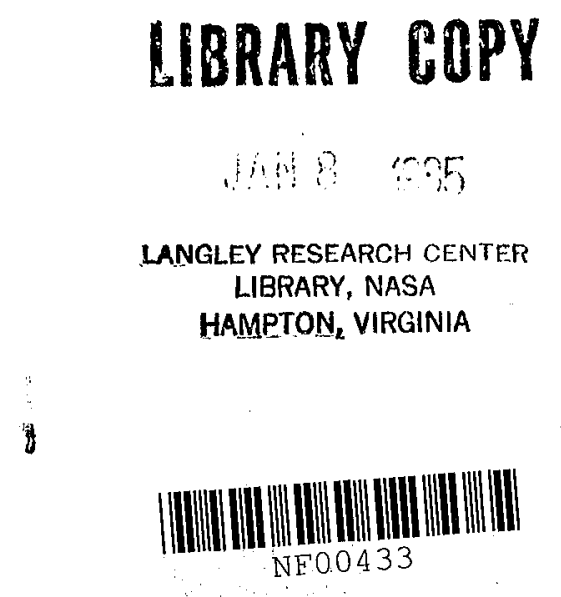

FFNo 672 Aug 65

$\mathrm{NE} 0.0433$ 


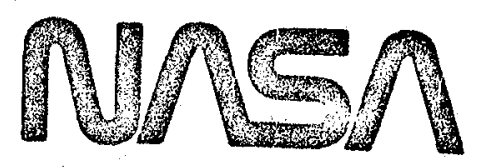

THE ANNULAR FLOW ELECTROTHERMAL RAMJET

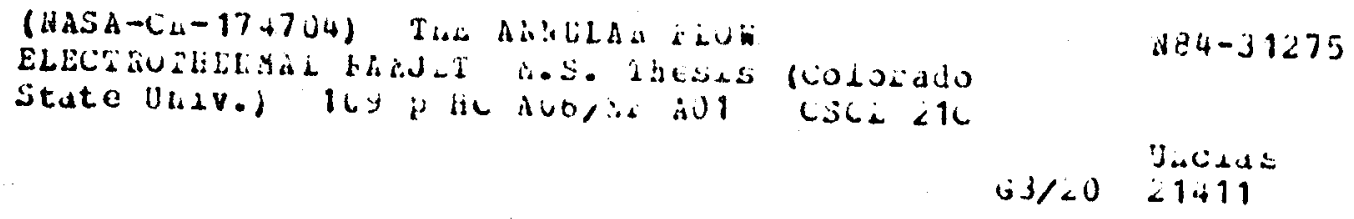

PREPARED FOR

LEWIS RESEARCH CENTER

NATIONAL AERONAUTICS AND SPACE ADMINSTRATION

GRANT NGR-06-002-112

by

Ben D. Shaw

Approved by

Paul J. Wilbur

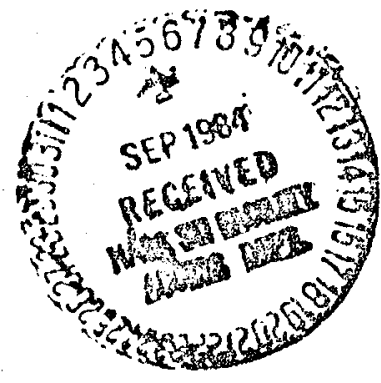

July 1984

Department of Mechanical Engineering

Colorado State University

Fort Collins, Colorado 


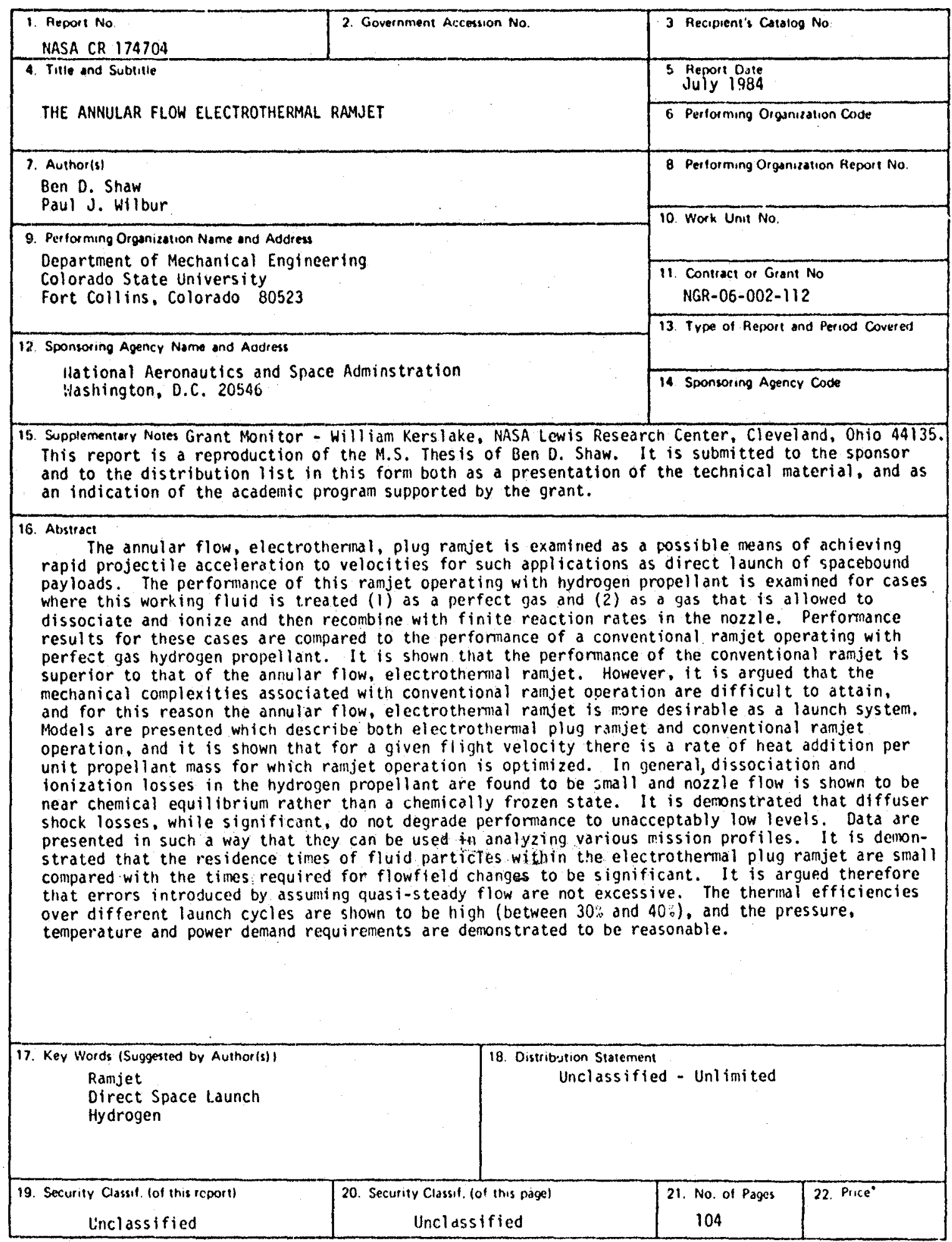

- For sale by the National Techurcal Inlounation Service. Sprughleid. Vuguna 22161 
TABLE OF CONTENTS

Page

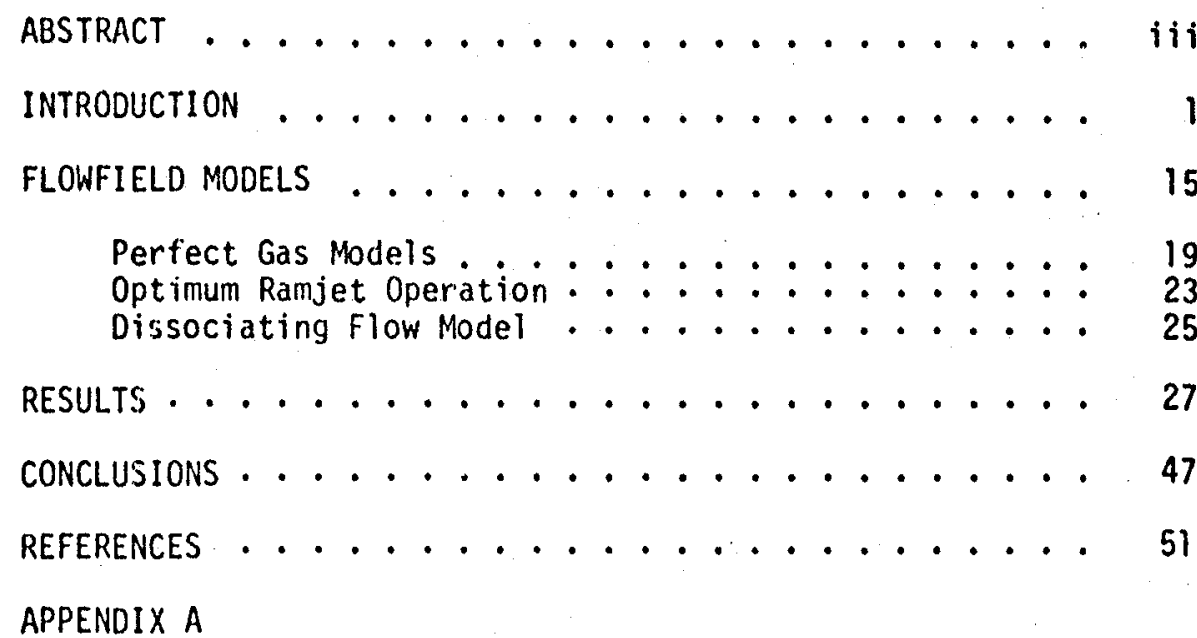

Conical Flowfield Averaging ......... 52 APPENDIX B

Equilibrium Hydrogen Properties . . . . . 62 APPENDIX C

Nonequilibrium Nozzle Flow.......... 88 APPENDIX 0

Unsteady Gas Dynamics Effects . . . . . . 96 
LIST OF FIGURES

Figure

Page

Fig. 1 Electrothermal Ramjet Concept . . . . . . . 2

Fig. 2 Typical Electrothermal Ramjet Launch Profile .. 5

Fig. 3 Ramjet Configurations ........... 16

Fig. 4 Instantaneous Efficiency Profiles....... 29

Fig. 5 Mass Fraction of $\mathrm{H}_{2}$ in Exhaust ....... 31

Fig. 6 Pressure-Induced Thrust Fraction Profiles . . . 32

Fig. 7 Outlet/Inlet Pressure Ratio Profiles. . . . . 34

Fig. 8 Outlet/Inlet Velocity Ratio Profiles . . . . 35

Fig. 9 Outlet/Inlet Static Temperature Profiles ... 37

Fig. 10 Instantaneous Efficiency Profiles at High Velocity 39

Fig. 11 Instantaneous Efficiency Profiles at Low Velocity 40

Fig. 12 Non-Dimensional Thrust Profile . . . . . 42 for Maximum Efficiency

Fig. 13 Maximum Instantaneous Efficiency Profiles.... 43

Fig. 14 Efficiency Profiles over Typical Launch Cycles. . 45

Fig. Al Electrothermal Ramjet Diffuser ....... 55

Fig. A2 Conical Flow Coordinate System ....... 59

Fig. B1 Equilibrium Hydrogen Composition at $10 \mathrm{Atm}$... 84

Fig. B2 Equilibrium Hydrogen Composition at $100 \mathrm{Atm}$. . 85

Fig. B3 Equilibrium Hydrogen Composition at $1000 \mathrm{Atm}$. . 86

Fig. Dl Electrothermal Ramjet Dimensions. ....... 99 


\section{NOMENCLATURE}

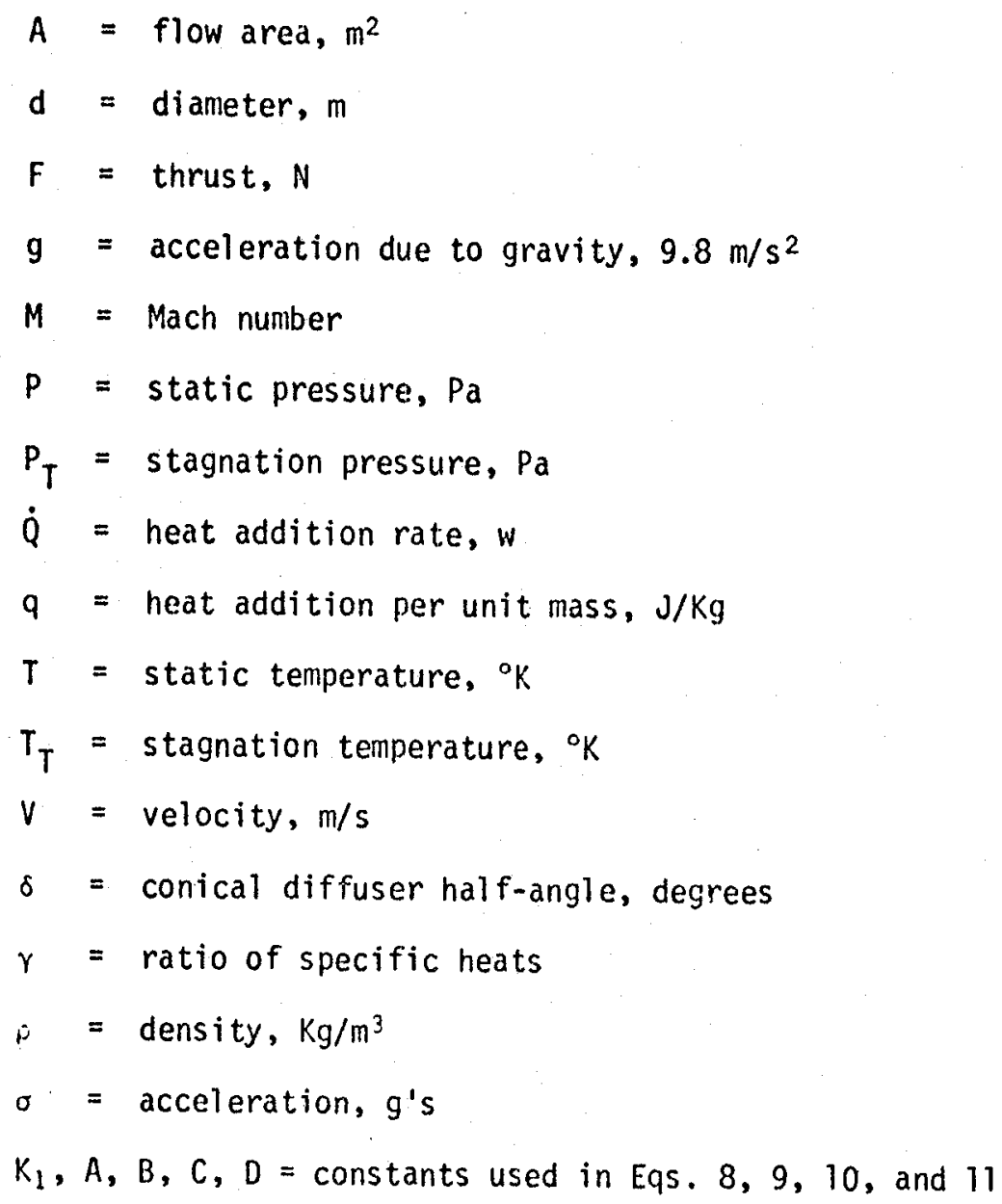




\section{INTRODUCTION}

Direct launch systems are currently being considered for space resupply and disposal missions where high accelerations can be tolerated. Payloads delivered into space with these systems would be accelerated to very high velocities (on the order of $10^{4} \mathrm{~m} / \mathrm{sec}$ ) close to the earth's surface and they would then be slowed somewhat to the desired final energy during passage through the earth's atmosphere. One such system that has been proposed recently for such missions is the electrothermal ramjet. ${ }^{1}$ This system appears to be capable of achieving the high acceleration levels needed to assure launch tracks that are of a reasonable length primarily because it does not accelerate either the propellant or the power plant required to effect its operation.

Conceptually the electrothermal ramjet involves a long tube filled with gaseous propellant through which a ramjet engine is accelerated to a specified terminal velocity. Typically the ramjet would be operated at constant thrust using hydrogen propellant heated by either electromagnetic radiation or electrical currents as it passes through the ramjet. Energy would either be beamed or conducted to the ramjet heat addition zone from the tube walls as the ramjet passed down the launch tube. Because a ramjet requires a substantial initial velocity before it can begin to produce thrust it is anticipated that the ramjet would be accelerated to this velocity by a device such as a light gas gun. Preliminary analysis ${ }^{1,2}$ has suggested that a particular ramjet design 


\section{ORIGINAL PAGE 19 \\ OF POOR QUALITY}

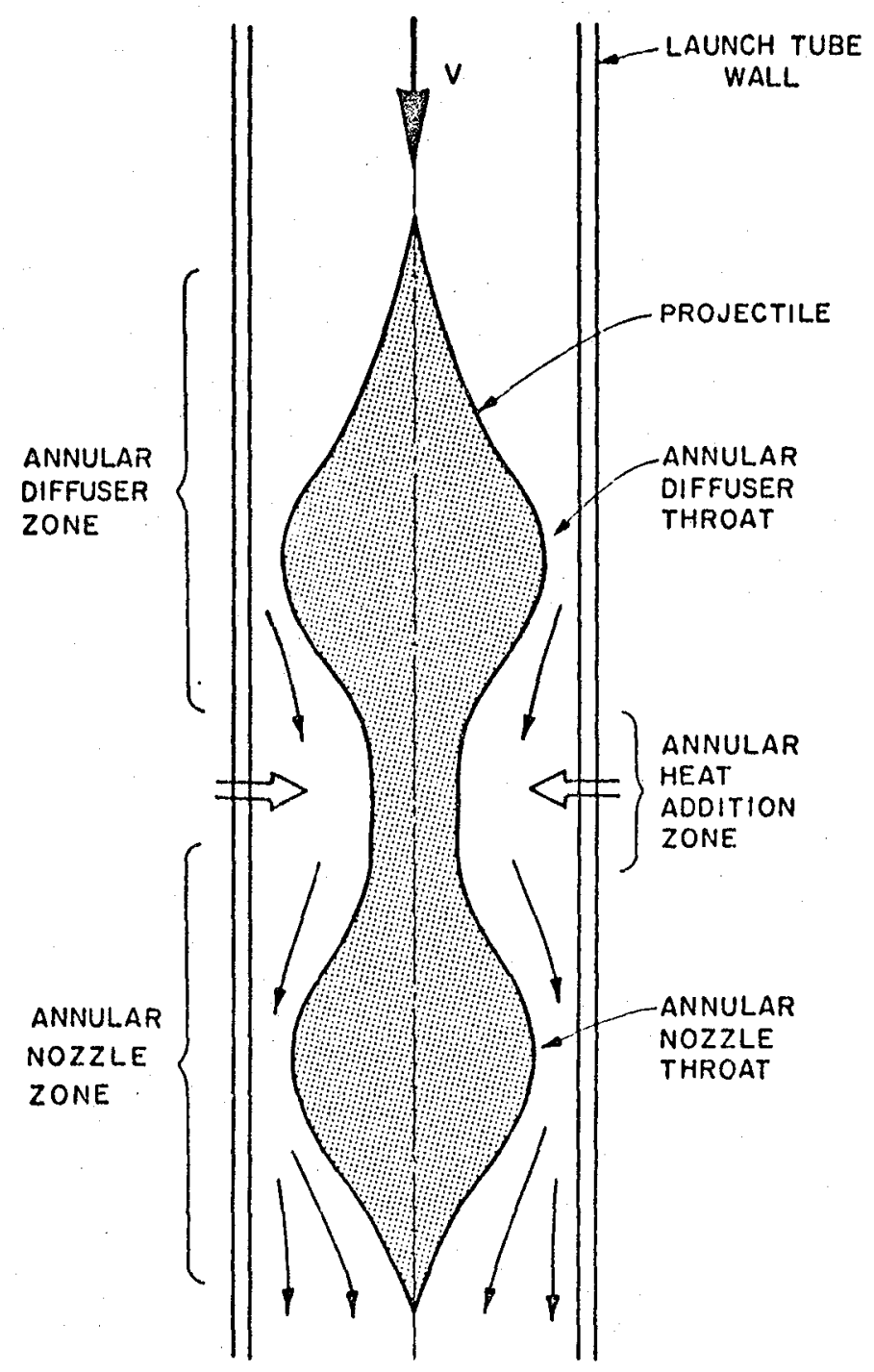

Fig. 1 Electrothermal Ramjet Concept 
referred to as the annular flow, plug ramjet is well suited to this application and that such a device should exhibit good performance.

The general concept of the electrothermal plug ramjet is shown in Fig. 1. The projectile, which is a solid centerbody, is shown traveling down the circular tube. The reference frame is chosen to be moving with the projectile so the gaseous propellant is shown moving towards the projectile with velocity $V$. The incoming propellant will encounter the annular diffuser where it will be slowed relative to the projectile. The coupling of the tube wall with the projectile forebody will produce the geometric configuration necessary to bring the propellant to subsonic conditions at the exit of the annular diffuser zone. After the propellant has been brought to subsonic conditions, heat is added in the annular heat addition zone, and the propellant then passes through the annular nozzle zone, which is a converging-diverging channel, and is expelled out the rear of the device so as to produce a thrust force on the projectile. This annular design is utilized because it has several attractive features including

1) Heat addition can be effected outside the ramjet body directly from the tube wall. This eliminates the need for windows in the ramjet body for the case of beamed energy heating and sliding current contacts for arc discharge heating schemes.

2) Direct mechanical or fluid mechanical forces induced between the tube wall and the projectile should tend to stabilize and center it.

3) The structural design of the projectile appears to be relatively simple.

4) Shock losses in the diffuser can be minimized by properly 
designing the annular diffuser section.

5) There will be no "spill over" of propellant.

6) Variations in fluid flow areas needed to achieve proper flow conditions as the projectile velocity increases can be achieved by varying the cross-sectional area of the launch tube as a function of axial position.

Praliminary analyses regarding annular flow electrothermal ramjet operation have been conducted ${ }^{1,2}$ and typical results obtained from these studies showing design and operating parameters for a typical missicn are presented in Figure 2. These results assume that there are no diffuser shock losses, that the hydrogen propellant behaves as a perfect gas, and that the fluid flow is quasi-steady. These results detail the perforinance of a ramjet launch cycle for the following specific operational parameters

1) A constant projectile mass of $10 \mathrm{~kg}$.

2) A constant acceleration of $30,000 \mathrm{~g}$ 's (hence constant thrust) from rest to $15,000 \mathrm{~m} / \mathrm{s}$.

3) An initial propellant temperature of $300^{\circ} \mathrm{K}$.

4) An initial propellant pressure of $30 \mathrm{~atm}$.

5) A fixed projectile diffuser throat diameter of $16 \mathrm{~cm}$ (Fig. 1).

6) A fixed projectile heat addition zone diameter of $8 \mathrm{~cm}$ (Fig. 1). If one assumes that the projectile will be acted upon by either the ramjet force or some externally applied force needed to produce the desired acceleration, the projectile trajectory will be known. With the projectile velocity profile known, the propellant flow velocity relative to the ramjet is known at each position along the launch track. One can now prescribe the diffuser throat area required at each position along 

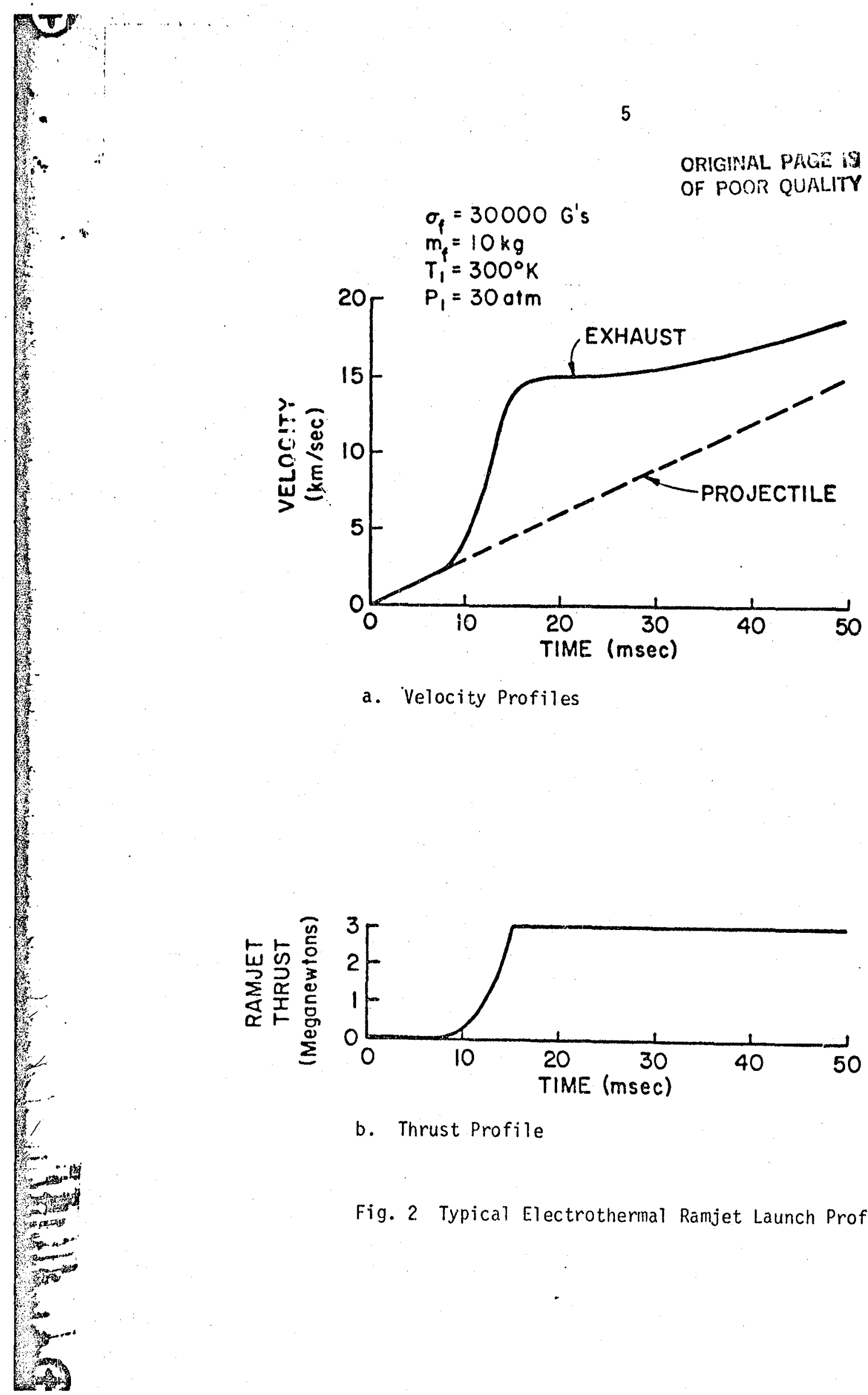

a. Velocity Profiles

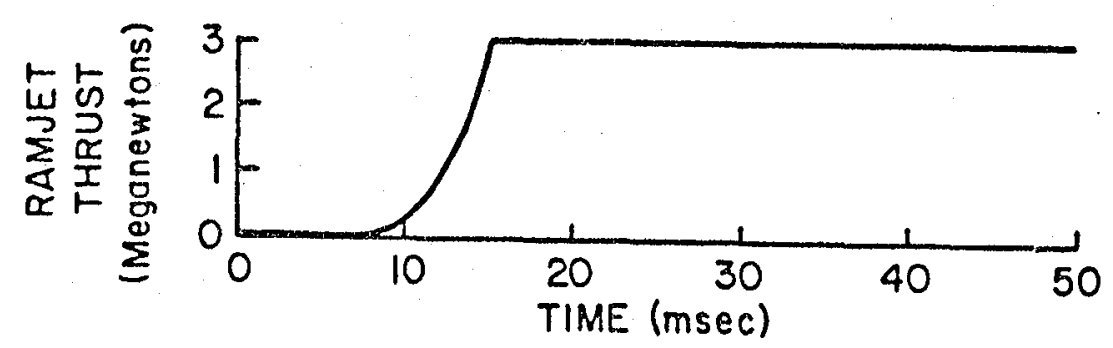

b. Thrust Profile

Fig. 2 Typical Electrothermal Rámjet Launch Profile 


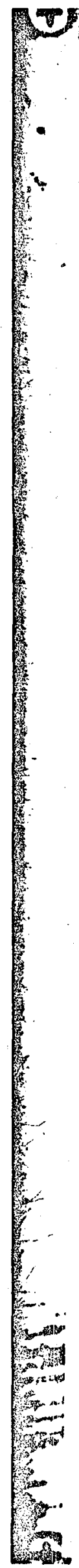

\section{6}

ORTCHSH Ran

OF POOR Gualoi:?

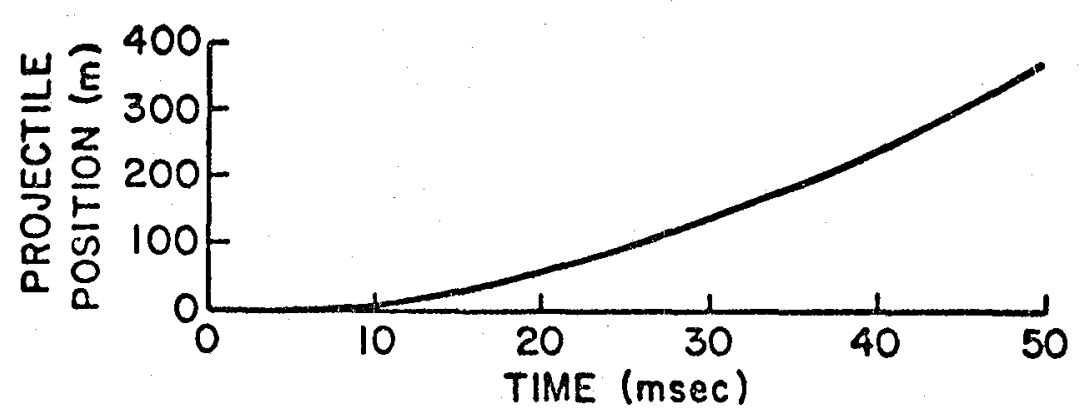

c. Projectile Position Profile

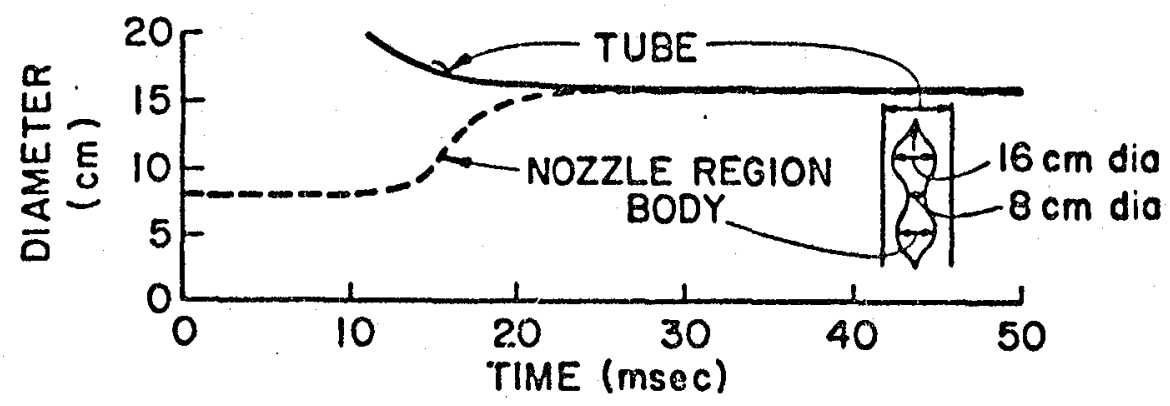

d. Projectile and Tube Diameter Profiles

Fig. 2 Typical Electrothermal Ramjet Launch Profile 


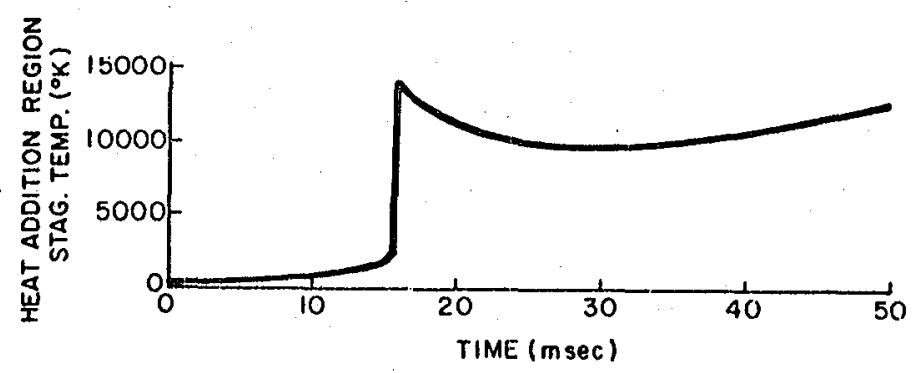

e. Heat Addition Stagnation Stagnation Temperature Profile

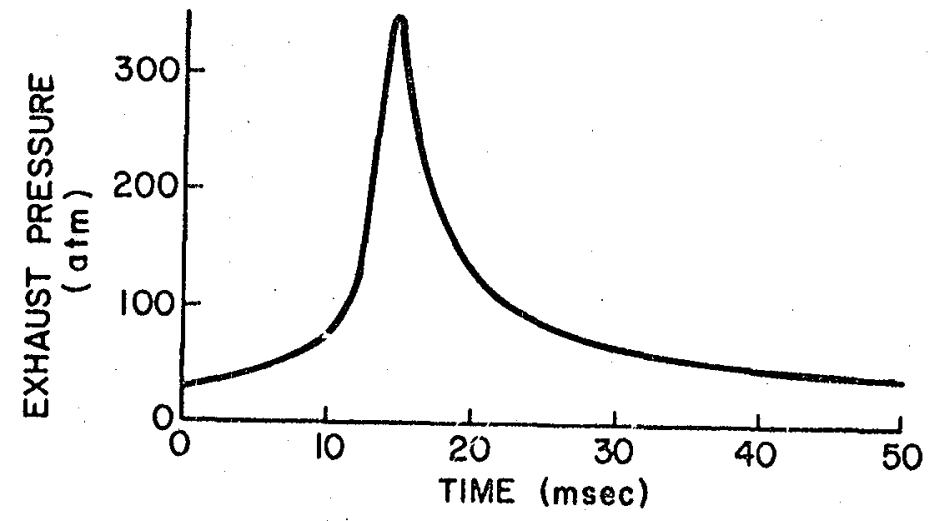

f. Exhaust Static Pressure Profile

Fig. 2 Typical Electrothermal Ramjet Launch Profile 


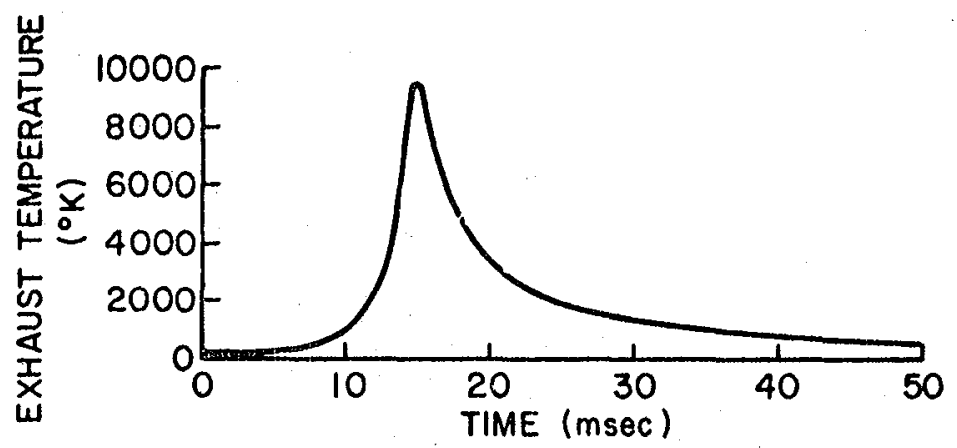

g. Exhaust Static Temperature Profile

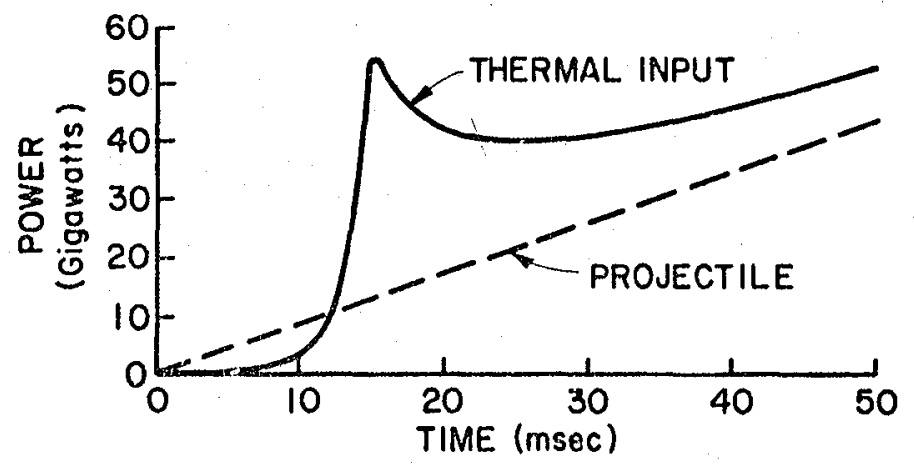

h. Thermal Input and Power Into Projectile Acceleration Profiles

Fig. 2 Typical Electrothermal Ramjet Launch Profile 
the tube so that the flow will remain sonic at this throat. One can also prescribe the heat supplied in the subsonic heat addition region so that the specified thrust will either be produced or the Mach number at the exit of the heating region (in the projectile frame of reference) reaches its maximum allowable value of unity. After heat has been added, the flow is accelerated isentropically in the nozzle to the maximum supersonic Mach number achievabie under the constraint of the area change available. The static pressure at the nozzle exit is determined by this expansion and it will vary with projectile position. Proper control of this pressure at the base of the projectile would have to be achieved in an actual device by proper design of the launch tube. Such a tube would consist of two sections: a cavity section into which hydrogen processed by the ramjet would be collected, and the launch section through which the projectile would pass. It has been assumed in this analysis that the cavity section could be designed to have the length and cross-sectional area needed to accommodate the propellant and facilitate maintenance of the necessary projectile base pressure. While it seems possible to do this intuitively, the examination of the unsteady fluid mechanics describing the process has not yet been attempted.

Figure 2 shows the results of such an analysis for the case where a constant thrust of $3 \times 10^{6} \mathrm{~N}$ is maintained on a $10 \mathrm{~kg}$ mass. As Fig. 2a shows, the projectile velocity (in the launch tube frame of reference) increases linearly with time. Since the propellant is initially at rest relative to the tube wall the projectile velocity is equal to the propellant inlet velocity in the projectile frame of reference. The exhaust velocity (in the projectile frame of reference) is observed to be equal to the inlet velocity during the first $\sim 8 \mathrm{~ms}$ of operation, so 
during this time interval, essentially no net thrust is produced through the mechanism of momentum change of the propellant. After about 16 ms of operation, however, the exhaust velocity has risen to about $15 \mathrm{~km} / \mathrm{s}$ and has begun to level off. During the time between $\sim 8$ and $\sim 16 \mathrm{~ms}$, all of the heat that can be added without choking the flow (achieving unity Mach number at the end of the heat addition region) is being added.

As Fig. $2 b$ shows, the net ramjet thrust is inadequate to meet the $3 \times 10^{6} \mathrm{~N}$ thrust requirement at times less than $16 \mathrm{~ms}$. Thus an al ternative accelerating scheme, such as the light gas gun ${ }^{3}$ would have to be used during this time interval. As the projectile velocity increases, more heat can be added and this causes the increase in exhaust velocity shown in Fig. 2a. After $\sim 16 \mathrm{~ms}$, both the exhaust velocity and the mass flow rate have risen to the point where the desired thrust ievel can be produced and the Mach number after heat addition begins to drop below unity.

Figure 2c, which irdicates projectile position as a function of time for this case of constant acceleration. shows a launch tube about $400 \mathrm{~m}$ long would be required to accelerate the $10 \mathrm{~kg}$ mass to the terminal velocity of $15,000 \mathrm{~ms}$ in $\sim 50 \mathrm{~ms}$. Figure $2 \mathrm{~d}$ shows the variation in tube and projectile diameters that would have to be effected to satisfy fluid mechanical constraints imposed on the ramjet. As previously stipulated, the projectile diameters at the diffuser throat and in the heat addition region have been treated as fixed at 16 and $8 \mathrm{~cm}$ respectively. The reduction in tube diameter required to yield unity Mach number at the diffuser throat as the projectile velocity increases is shown by the solid line. Data from this line taken together with the position 
profile of Fig. $2 c$ specify how the tube diameter would have to vary along its length. Figure $2 d$ also shows that the projectile diameter at the nozzle throat would have to increase as the projectile velocity increases and the input power is varied to induce the desired thrust level. The fact that this diameter must change during the launch cycle represents a significant mechanical complication in the system. It is noted, however, that the amount of change in this diameter becomes very small after about $20 \mathrm{~ms}$ when the projectile velocity is $\sim 5 \mathrm{~km} / \mathrm{s}$. It is anticipated that a pressure- or temperature-sensitive scheme might be designed to facilitate this change in projectile diameter at the nozzle throat. Alternatively, one could hold the projectile diameter in the nozzle region fixed, reduce the tube diameter slightly more rapidly than Fig. $2 d$ suggests, and abrade the diameter of the projectile at the diffuser throat using protrusions from the tube wall.

Figures $2 \mathrm{e}, 2 \mathrm{f}$, and $2 \mathrm{~g}$ indicate how the stagnation temperature in the heat addition zone and the static pressure and temperature at the nozzle exit should vary to conform to the problem specifications. The peaks in temperature and pressure occur at the point where the power input is a maximum (Fig. $2 h$ ). This, in turn, occurs at the time when the thrust has just reached its design value and the Mach number at the end of the heat addition region is still at unity. At this condition, the exhaust velocity is still relatively low and the bulk of the thrust is being produced by the static pressure difference across the projectile. If one $d$ d not start using the ramjet until after $\sim 20 \mathrm{~ms}$ into the launch, the exhaust pressure and temperature would lie in a very moderate range throughout the period of ramjet operation. Note that it is the pressure profile of Fig. $2 f$ that would have to be matched 
through proper design of the cavity section of the launch tube. This might be achieved by selecting not only the length and cross-sectional area of this section, but also by the inclusion of accumulator chambers that could be connected into the tube at the proper time.

The heat addition zone stagnation temperature (Fig. 2e) is observed to remain near $10,000^{\circ} \mathrm{K}$ after about $16 \mathrm{~ms}$. It is noted that, dissociation and ionization may introduce significant effects while operating within this regime, and these effects will be examined later in this analysis.

Figure $2 h$ shows the thermal power input profile required to effect operation as well as the actual profile of mechanical power input to the projectile. After the $\sim 20 \mathrm{~ms}$ period over which the auxiliary starting force would be applied, it is observed that the ratio of projectile power to thermal input power rises above $\sim 50 \%$. The ramjet is observed to become increasingly efficient as the launch cycle proceeds. This suggests that the ramjet will be more efficient at the higher velocities, where the efficiencies of electromagnetic launchers drop off. The difference between the projectile and thermal input powers represents the power being deposited as kinetic and thermal energy in this hydrogen propellant. It should be noted here that frozen flow losses in the nozzle and electrical-to-thermal power conversion losses as well as frictional losses have been neglected in this preliminary analysis.

The results presented in Fig. 2 suggest that the annular flow electrothermal ramjet concept has the potential of achieving efficient high velocity high acceleration launches, and also that this concept cannot be rejected on the basis that it violates a basic principle of fluid mechanics or thermodynamics. The pressure, temperature and power 
demand profiles are all reasonable, and it is for these reasons that further analysis of the annular flow electrothermal ramjet was undertaken to bring to light aspects which may degrade ramjet performance.

There are several effects which may degrade plug ramjet performance below that shown in Figure 2. Some of these effects are

1) Diffuser shock losses.

2) Propellant dissociation, ionization and non-perfect modes of molecular and atomic energy storage.

3) Freezing (translational, rotational, vibrational, chemical).

4) Non-steady gas dynamics effects.

It is the purpose of this thes is to address these effects and to determine the magnitude of degradation of annular flow electrothermal ramjet performance that they might cause.

In order to illustrate the performance of the electrothermal plug ramjet, an analysis will be also conducted on the more familiar conventional ramjet which uses hydrogen propellant that behaves as a perfect gas. The results from this analys is will be compared to analyses conducted for the annular flow electrothermal plug ramjet. Simple theoretical models detailing conventional ramjet and electrothermal ramjet operation with perfect gas hydrogen propellant will be presented, and data from the more extensive analyses considering dissociation, ionization and non-equilibrium processes will be interpreted in the light of the results from the simplified models.

It should be stated here it has been assumed in all analyses that the fluid flow is axis-symmetric or one-dimensional and quasi-steady, and the fact that the tube wall is moving in the reference frame of the annular flow electrothermal plug ramjet is assumed to have no 
influence on the flow. All flowfields analyzed are considered to be inviscid. The ideal gas equation of state is assumed to be valid at all times. 


\section{FLOWFIELD MODELS}

The general concept of a conventional ramjet is shown in Fig. 3a. The reference frame is chosen to be moving with the ramjet, so the propellant is shown moving towards the inlet with a velocity $v_{1}$. The Mach number associated with the incoming flow is designated $M_{1}$. After the ramjet has been accelerated to a high enough initial velocity, an ob1 ique shock will be attached to the conical tip of the center body which serves as a diffuser. Downstream of the diffuser tip, a series of shocks reflected between the center and outer bodies will occur. They will terminate in a normal shock that will slow the flow to subsonic conditions. A review of the hypersonic wind tunnel literature 4 has suggested that normal shock recovery can be expected after the initial conical shock, so it is assumed that the flow passes through an initial conical shock to state 2 and then passes through a single normal shock to state 3 . One-dimensional values sbtained by averaging over the axis-symmetric conical flow field variables computed at the exit to the conical flow field in Region 2 are used as input for the normal shock relations. For a description of the methods used to compute the ayerage conical flowfield values see Appendix $A$.

Across the normal shock, equations describing mass continuity, the first law of thermodynamics, conservation of momentum, and the second law of themodynamics are satisfied. Immediately downstream of the normal shock it is assured that the area between the center and outer 
ORIGINAL PRG: : :

OF POOR QUALMT

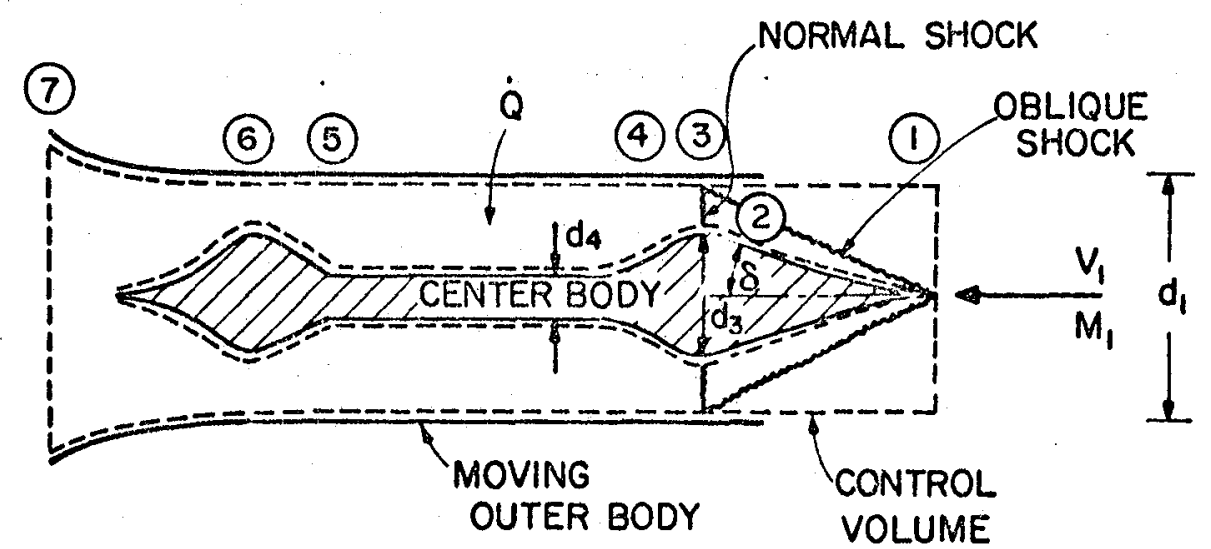

a. CONVENTIONAL RAMUET

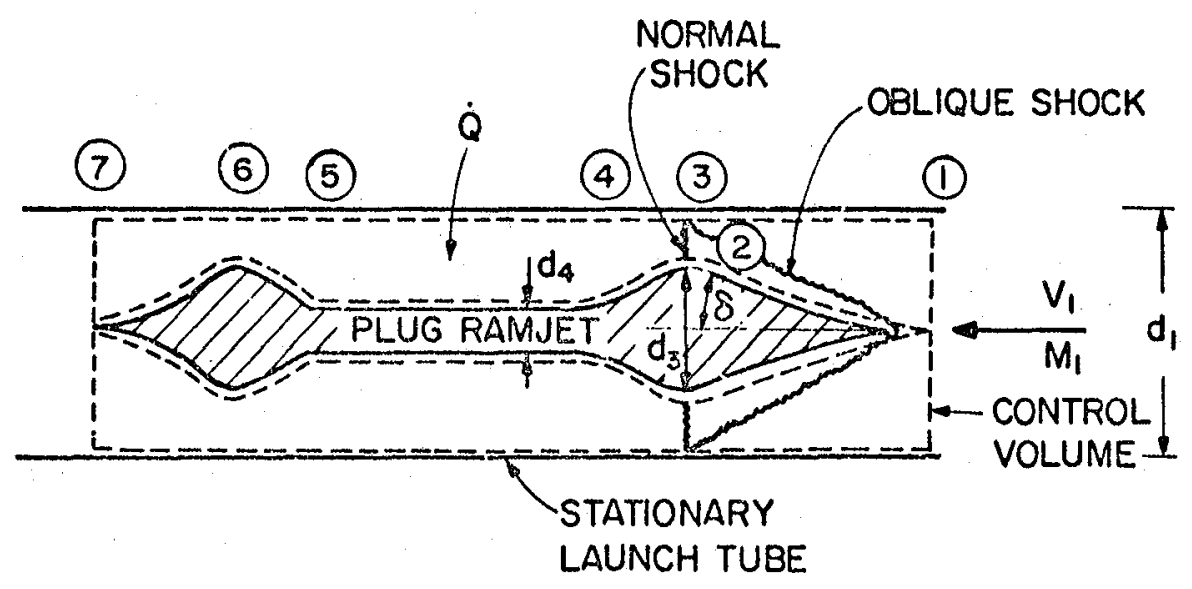

b. ELECTROTHERMAL ANNULAR FLOW RAMJET

Fig. 3 Ramjet Configurations 
bodies is sufficient so the flow will pass through the minimum area that is encountered at the diffuser throat. After the normal shock, the flow enters the subsonic heat addition region at 4 and heat is assumed to be deposited into the flow between States 4 and 5 . In the heat addition region, the first and second laws of thermodynamics are satisfied, along with mass continuity and the momentum equation. After leaving the heat addition region at 5 , the flow passes through a converging-diverging channel where it is accelerated first to the sonic condition at state 6 and then on to supersonic conditions at the nozzle exit 7 .

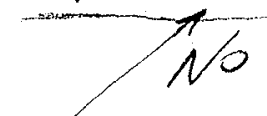

With this conventional ramjet (Figure $3 a$ ) the static inlet and outlet pressures are equal because the free stream conditions are communicated outside of the moving outer body. In order to satisfy this condition while assuming no shocks in the nozzle one finds the nozzle exit area $\left(A_{7}\right)$ must be larger than the free-flow diffuser inlet area $\left(A_{1}\right)$. It is hoped that by comparing this more familiar ramjet to the new one being proposed that the concept and performance of the plug ramjet can be better understood.

The annular flow plug ramjet is shown in Fig. 3b. It appears similar to the conventional ramjet at first glance, but is different in the following ways

1) The plug ramjet moves down the tube as the center body of the conventional ramjet does but in the plug ramjet case the outer body is the launch tube which is stationary. Because the launch tube diameter is essentially constant along its length the fluid mechanics and thermodynamics of the problem can be considered from the frame of reference of the ramijet as though 
this tube were not moving.

2) The inlet and outlet areas associated with flow through the plug ramjet $\left(A_{1}\right.$ and $\left.A_{7}\right)$ must be equal, again because the launch tube diameter does not vary significantly along its length.

3) The inlet and outlet pressures $P_{1}$ and $P_{7}$ are not equal because the upstream and downstream regions are not connected through an exterior path. In fact the outlet pressure $P_{7}$ will be greater than the inlet pressure $P_{1}$ and this pressure difference will therefore produce some component of the thrust exerted on the plug.

One can see that the fluid mechanics of both configurations in Figs. $3 a$ and $3 b$ are described by the same basic equations. The only difference in the two cases is the difference in the boundary conditions that are applied to the flow.

When non-perfect gas modes of energy storage such as ionization and dissociation are allowed to occur, results from statistical mechanics are utilized to compute equilibrium compositions and thermodynamic properties and shifting chemical composition is allowed to occur. For a description of the methods used to compute the equilibrium properties of hydrogen see Appendix B. Where they are deemed potentially important, nonequilibrium processes are evaluated by considering finite rate reactions and by calculating the conditions at which specific reactions will become slow enough so the chemical composition of the flow becomes essentially frozen. A description of the methods utilized to perform these calculations can be found in Appendix $C$.

Because the essense of the phenomena occurring in ramjet devices of the types considered here can be described by simple, perfect gas 
models, these types of theoretical models will be presented to illustrate the basic operational trends present in the analyses conducted. Perfect Gas Models

Consider now that either ramjet in Fig. 3 is being accelerated at a constant thrust $F$ and that heat is being added at a rate $\dot{Q}$ to achieve this level of thrust. In general $\dot{Q}$ will vary with the velocity of the ramjet and will be different for the conventional and plug designs. By assuming that the propellant flowing through the control volumes shown in Figs. 3a and $3 b$ behaves as a perfect gas, the momentum equation applied across the control volumes of Fig. 3 can be put in the following form

$$
\frac{F}{P_{7} A_{1}}=\frac{A_{7}}{A_{1}} \frac{P_{T_{7}}}{P_{T_{1}}}\left[\frac{1+\frac{\gamma-1}{2} M_{1}^{2}}{1+\frac{\gamma-1}{2} M_{7}^{2}}\right]^{\frac{\gamma}{\gamma-1}}\left[\gamma M_{7}^{2}+1\right]-\left[\gamma M_{1}^{2}+1\right] .
$$

In this equation the entrance and exit conditions are respectively; Mach number $M_{1}, M_{7}$; area $A_{1}, A_{7}$ and stagnation pressure $P_{T_{1}}, P_{T_{7}}$. The ratio of specific heats is $\gamma$ and $P_{1}$ represents the entrance static pressure. The left hand side of this equation represents a non-dimensionalized thrust. The stagnation pressure ratio appearing in Eq. 1 can be written

$$
\frac{\mathrm{P}_{T_{7}}}{\mathrm{P}_{\mathrm{T}_{1}}}=\frac{\mathrm{P}_{\mathrm{T}_{4}}}{\mathrm{P}_{\mathrm{T}_{1}}} \frac{\mathrm{P}_{\mathrm{T}_{5}}}{\mathrm{P}_{\mathrm{T}_{4}}} \frac{\mathrm{P}_{\mathrm{T}_{7}}}{\mathrm{P}_{\mathrm{T}_{5}}}
$$

where $\left(\mathrm{P}_{\mathrm{T}_{4}} / \mathrm{P}_{\mathrm{T}_{1}}\right)$, the stagnation pressure ratio across the diffuser, is less than unity because of diffuser losses. The heat addition region stagnation pressure ratio $\left(\mathrm{P}_{\mathrm{T}_{5}} / \mathrm{P}_{\mathrm{T}_{4}}\right)$ is also less than unity because of heat addition-induced stagnation pressure losses. Because the nozzle is assumed to be isentropic the ratio $\left(P_{T_{7}} / P_{T_{5}}\right)$ is unity. 
When the first law of thermodynamics is applied to a perfect gas working fluid, the following expression for the instantaneous ramjet efficiency (power into ramjet mass acceleration divided by input thermal power) is obtained

$$
\frac{F V_{1}}{\dot{Q}}=\frac{F}{P_{1} A_{1}} \frac{\gamma-1}{\gamma}\left[\left(1+\frac{\gamma-1}{2} M_{1}^{2}\right)\left(\frac{T_{T_{7}}}{T_{T_{1}}}-1\right)\right]^{-1} .
$$

To apply these equations to the analysis of either ramjet it is necessary to specify the inlet Mach number $\left(M_{1}\right)$ and the diffuser geometry so that the diffuser stagnation pressure ratio $\left(\mathrm{P}_{T_{4}} / \mathrm{P}_{\mathrm{T}_{1}}\right)$ can be computed. For a given conical diffuser half-angle $(\delta)$ and annular diffuser shoulder diameter $\left(d_{3}\right)$ and heat addition center body diameter $\left(d_{4}\right)$ the inlet free-flow diameter $\left(d_{1}\right)$ is specified by placing the iritersection of the conical shock and the outer body or tube wall at the annular diffuser shoulder. Specifying these parameters serves to fix the flow conditions up to State 4 , where subsonic conditions are always specified.

The flow downstream of the conical flowfield is assumed to be onedimensional, so one-dimensional values for the conical flowfield variables are computed by the methods detailed in Appendix A. These onedimensional values of the variables are then used as input to the normal shock relations so the stagnation pressure ratio $\mathrm{P}_{T_{4}} / \mathrm{P}_{T_{1}}$ and the flow conditions downstream of the normal shock at 3 can be utilized to specify the conditions at the entrance to the heat addition region 4 . By knowing $M_{4}$ and specifying $M_{5}$, the haät addition stagnation pressure ratio $\left(P_{T_{5}} i P_{T_{4}}\right)$ is fixed, as is the stagnation temperature ratio $\left(T_{T_{5}} / T_{T_{4}}=T_{T_{7}} / T_{T_{1}}\right)$. To analyze ramjet operation then, either the 
exit/inlet area ratio $\left(A_{7} / A_{1}\right)$ or the exit/inlet static pressure ratio $\left(P_{7} / P_{7}\right)$ may be specified to fix $M_{7}$. For the conventional ramjet the pressure ratio $P_{7} / P_{1}$ is set to unity, and for the electrothermal ramjet the area ratio $A_{7} / A_{1}$ is set to unity. It is noteworthy that the parameters $A_{7} / A_{1}, P_{7} / P_{1}$ and $M_{7}$ are not completely independent. Specifying one parameter dictates values for the others.

Dictating the exit/inlet conditions for the ramjet. control volume allows the calculation of other parameters such as the exit/inlet velocity and temperature ratios, and the fractions of the total thrust provided by 1) the difference in the pressure area product between the entrance and exit flowfields and 2) the momentum change in the propellant. The mathematical relationships utilized to compute these ratios and fractions are not shown, but graphical results are illustrated in the Results Section of this thesis for specific operating conditions.

To further illustrate the performance of the ramjets operating with a perfect gas propellant it is instructive to express the first law of thermodynamics in the form

$$
\frac{V_{7}}{V_{1}}=\left(\frac{T_{T}}{T_{T_{1}}}\right)^{\frac{1}{2}}\left\{\frac{2}{M_{1}^{2}(\gamma-1)}\left[1-\left(\frac{P_{T_{7}}}{P_{T_{1}}} \frac{1}{P_{7}}\right)^{\frac{1-\gamma}{\gamma}}\right]+1\right\}^{\frac{1}{2}}
$$

and the momentum equation in the form

$$
\frac{F}{\dot{m} V_{1}}=\left(\frac{V_{7}}{V_{1}}-1\right)+\frac{1}{M_{1}^{2} \gamma}\left\{\frac{T_{7}}{T_{T_{1}}}\left(\frac{P_{T_{7}}}{P_{T_{1}}} \frac{P_{1}}{P_{7}}\right)^{\frac{1-\gamma}{\gamma}} \frac{1}{\left(V_{7} / V_{1}\right)^{-1}}\right\} \text {. }
$$

Equation 5 can be multiplied by the quantity 


$$
\frac{v_{1}^{2}}{\frac{{ }^{2}}{\gamma-T} R T T_{1}\left(\frac{T_{T_{7}}}{T_{T_{1}}}-1\right)}
$$

to obtain the form

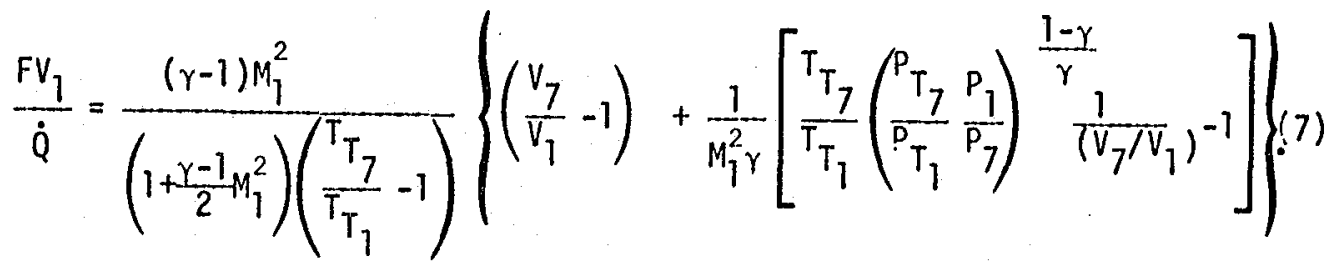

For the conventional ramjet

$$
\begin{aligned}
& \text { nut zecess arty } \\
& \text { cil snguesm }
\end{aligned}
$$$$
P_{7} / P_{1}=1 .
$$

The stagnation pressure ratio $\mathrm{P}_{T_{7}} / \mathrm{P}_{\mathrm{T}_{1}}$ is given by

$$
\frac{\mathrm{P}_{T_{7}}}{\mathrm{P}_{\mathrm{T}_{1}}}=\frac{\mathrm{P}_{\mathrm{T}_{4}}}{\mathrm{P}_{\mathrm{T}_{1}}} \frac{\mathrm{P}_{5}}{\mathrm{P}_{\mathrm{T}_{4}}} \frac{\mathrm{P}_{\mathrm{T}_{7}}}{\mathrm{P}_{\mathrm{T}_{5}}}
$$

where $P_{T_{4}} / P_{T_{7}}$ is fixed by the inlet flow conditions and the diffuser geometry and $\mathrm{P}_{\mathrm{T}_{7}} / \mathrm{P}_{\mathrm{T}_{5}}$ is unity. The stagnation pressure ratio $\mathrm{P}_{T_{5}} / \mathrm{P}_{\mathrm{T}_{4}}$ is dictated by the rate of heat addition per unit mass to the propellant and for subsonic heat addition this ratio does not vary appreciably. Examination of Eq. 4 shows that for fixed values of $M_{7}, r, P_{T_{4}} / P_{T_{1}}, P_{7} / P_{7}$ and $P_{T_{7}} / P_{T_{5}}$, the effect of the variation of $P_{T_{5}} / P_{T_{4}}$ on the expression

$$
\left\{\frac{2}{M_{7}^{2}(\gamma-1)}\left[1-\left(\frac{{ }_{T_{7}}}{P_{T_{1}}} \frac{P_{1}}{P_{7}}\right)^{\frac{1-\gamma}{\gamma}}\right]+1\right\}^{\frac{1}{2}}
$$

is small compared to the change in 


$$
\left(\frac{T_{T_{7}}}{T_{T_{1}}}\right)^{\frac{1}{2}}
$$

as the rate of heat addition per unit mass is varied. As a result, Eq. 4 <an be written

$$
\frac{v_{7}}{V_{1}} \equiv K_{1}\left(\frac{T_{T_{7}}}{T_{1}}\right)^{\frac{1}{2}}
$$

where $k_{1}$ is approxinately constant. The variation in $k_{1}$ is typically of the order of $5 \%$ for the cases considered.

\section{Optimum Ramjet Operacion}

It will be shown in the Results Section of this thesis that there is a value of the non-dimensional thrust $\left[F / P_{1} A_{1}\right]$ for which the instantaneous thermal efficiency $\left[F V_{1} / \dot{Q}\right]$ is at a maximum for a specified diffuser and given free-flow inlet conditions. An approximate mathematical relationship which predicts the point of maximum efficiency for the conventional ramjet operating with a perfect gas propellant can be derived by considering the first law of themodynamics and the momentum equation. The quantity appearing in Eq. 5

$$
\left(\begin{array}{ll}
P_{T_{7}} & P_{1} \\
P_{T_{1}} & \frac{1-\gamma}{P_{7}}
\end{array}\right)^{\frac{1-\gamma}{1}}
$$

varies by about $6.5 \%$ at the most, so it can be treated as a constant. By assuming that the previously mentioned quantities are constant Eq. 5 can be put into the following functional form 


$$
\frac{F}{m v_{1}}=A\left(\frac{T_{T_{7}}}{T_{T_{1}}}\right)^{\frac{1}{2}}-B
$$

where $A$ and $B$ are constants. This equation states that the ramjet thrust varies as the square root of the heat addition rate per unit mass. Similarly the expression for instantaneous efficiency (Eq. 7) can be put into the form

$$
\frac{F V_{1}}{\dot{Q}}=\frac{c\left(\frac{T_{T_{7}}}{T_{T_{1}}}\right)^{\frac{1}{2}}-0}{\frac{T_{T_{7}}}{T_{T_{1}}}-1}
$$

where $C$ and $D$ are constants.

Examination of $\mathrm{Eq} .10$ reveals that there is an optimum stagnation temperature ratio that will yield a maximum instantaneous efficiency. From Eq. 9 one sees that this optimum temperature ratio corresponds to an optimum normalized thrust level. This optimum corresponds to a ramjet characterized by specified geometry and inlet conditions. Changes in the inlet condition will necessitate changes in the opitimum stagnation temperature ratio and the optimum normalized thrust level. The values for the constants $C$ and $D$ are given by

$$
\begin{aligned}
& C=k_{2}\left(k_{1}+k_{3} k_{4}\right) \\
& D=K_{2}\left(1+k_{3}\right)
\end{aligned}
$$

where

$$
\begin{aligned}
& K_{1}=\left\{\frac{2}{M_{1}^{2}(\gamma-1)}\left[1-\left(\frac{{ }^{P} T_{7}}{P_{T_{1}}} \frac{P_{1}}{P_{7}}\right)^{\frac{1-\gamma}{\gamma}}\right]+1\right\}^{\frac{1}{2}} \\
& K_{2}=\frac{(\gamma-1) M_{1}^{2}}{\left(1+\frac{\gamma-1}{2} M_{1}^{2}\right)}
\end{aligned}
$$




$$
\begin{aligned}
& K_{3}=\frac{1}{M_{1}^{2} \gamma} \\
& K_{4}=\frac{\left[\left(P_{T_{7}} / P_{T_{1}}\right)\left(P_{1} / P_{7}\right)\right]^{\frac{1-\gamma}{\gamma}}}{K_{1}} .
\end{aligned}
$$

These relations are valid where $P_{1} / P_{7}$ is held to be constant and the stagnation pressure ratio $\mathrm{P}_{T_{5}} / \mathrm{P}_{\mathrm{T}_{4}}$ takes on typical values in the range 0.8 to 1.0. By differentiating the instantaneous efficiency (Eq. 10) with respect to the stagnation temperature ratio, the stagnation temperature ratio which gives the optimum instantaneous efficiency can be computed. The expression for this optimum ratio is

$$
\begin{aligned}
& \left(\frac{T_{T_{7}}}{T_{T_{1}}}\right)_{\mathrm{FV}}= \\
& \frac{\dot{Q}}{\dot{Q}}=\text { Maximum }
\end{aligned}
$$

\section{Dissociating Flow Model}

It was anticipated that assuming the propellant behaves as a perfect gas would introduce errors into these analyses, because it was recognized that effects such as vibrational and electronic excitation dissociation and ionization of the propellant would play significant roles in the performance of the ramjet device. The annular flow electrothermal plug ramjet was also analyzed with the above effects included. The validity of assuming either chemically frozen or equilibrium chemical composition in the supersonic nozzle expansion was also evaluated. In analyzing the flow in the diffuser for this dissociating flow case it was assumed that the fluid in the conical flowfield between the normal 
shock and the oblique shock behaved as a perfect gas and thus that this portion of the model developed for the perfect gas models could be applied. This assumption should not introduce too much error into this analysis because the oblique shock angle should be shallow for high inlet velocities and small diffuser cone angles, and the temperature rise in the conical flowfield should not be too large. Downstream of the normal shock, chemical relaxation and other non-perfect gas modes of energy storage were allowed to occur in the propellant. The propellant was assumed to be in thermodynamic equilibrium downstream of the normai shock and up to the annular nozzle throat (6). Finite chemical reaction rates were evaluated in the supersonic nozzle expansion between 6 and 7 . Because shifting chemical equilibrium was allowed, closed form equations for the thermodynamic properties could not be obtained. The method of analysis utilized for the model including shifting composition involved piece-wise application of the momentum and energy equations between each state identified in Fig. 3b that is downstream of the normal shock.

In order to conpare the performance of the perfect gas ramjet to that of the ramjet utilizing the chemically reacting propellant, the diffuser operating conditions are specified and the non-dimensional thrust is computed along with various other non-dimensional parameters such as the inlet/exit temperature and velocity ratios. Different values for the non-dimensional thrust $\left[F / P_{1} A_{1}\right]$ are determined by independently varying each component in the non-dimensiunal thrust equation, and the downstream flow conditions are found such that a specified, constant thrust level is met. 


\section{RESULTS}

It is instructive to examine a ramjet operating at a typical flight velocity that would be encountered at one point in a launch sequence so that the influences of the various operational parameters can be established. In order to do this several different ramjet configurations have been analyzed for a moderate flight velocity of $7000 \mathrm{~m} / \mathrm{s}$. A diffuser cone half-angle $(\delta)$ of $30^{\circ}$; a static temperature $\left(T_{1}\right)$ of $300^{\circ} \mathrm{K}$, and diffuser shoulder diameter-to-tube diameter $\left(d_{3} / d_{1}\right)$ and heat addition region diameter-to-tube diameter $\left(d_{4} / d_{1}\right)$ ratios of 0.805 and 0.403 respectively (Fig. 3 ), have been used in the analysis. The $30^{\circ}$ cone angle assured conical shock attachment and reasonable diffuser efficiency. The static temperature $T_{1}$ was selected as a reasonable value that could exist in an actual device. The diameter ratios resulted in adequate center body structural integrity and they were also such that choking at the diffuser throat was precluded. The specific ramjet configurations examined are (1) the conventional ramjet utilizing diatomic hydrogen which behaves as a perfect gas as a working fluid and with the constraint that the inlet and outlet static pressures are equal, (2) the annular flow electrothermal ramjet operating with the same perfect gas and with the constraint that the inlet and outlet flow areas are equal and (3) the annular flow electrothermal ramjet operating with hydrogen propellant that is allowed to dissociate and ionize and to have vibrational and electronic modes of energy storage along with finite 
chemical relaxation rates occurring in the supersonic nozzle expansion. For this latter case two thrust levels per unit cross sectional area of tube have been specified. The higher value for the thrust per unit area is $F / A_{1}=9.5 \times 10^{7} \mathrm{~Pa}$ and the lower value for the thrust per unit area is $F / A_{1}=9.5 \times 10^{6} \mathrm{~Pa}$. Two values have been considered so that the effect of varying the initial pressure in the launch tube can be illustrated for high and low thrust per unit area values.

Figure 4 illustrates the relationship between the instantaneous efficiency $\left[F V_{1} / Q\right]$ and the non-dimensional thrust $\left[F / P_{1} A_{1}\right]$ for the previously mentioned operational parameters. Increasing the nondimensional thrust implies increasing the heat addition per unit mass to the propellant. The perfect gas conventional ramjet is shown to exhibit the best performance, and the optimum value for instantaneous efficiency is seen to occur at a non-dimensional thrust of 12 for the conventional ramjet. This peak value is predicted to first order by Eq. 10.

The consequence of not expanding the supersonic nozzle flow to the pressure ratio $P_{7} / P_{7}$ of unity is illustrated in Fig. 4 by the curve for the electrothermal plug ramjet operating with a perfect gas (no dissociation). Its performance is seen to be degraded substantially with respect to the conventional ramjet operating with a perfect gas. The other curves, which illustrate the effects of chemical freezing, dissociation, ionization and vibrational and electronic modes of energy storage, are shown to exhibit performance which is similar but inferior to the electrothermal plug ramjet operating with a perfect gas. It is noted that the dissociating propellant curves of Fig. 4 were generated by fixing the thrust-to-cross sectional area ratio $\left(F / A_{1}\right)$ and varying the initial pressure in the launch tube $P_{1}$. The maximum value of the 


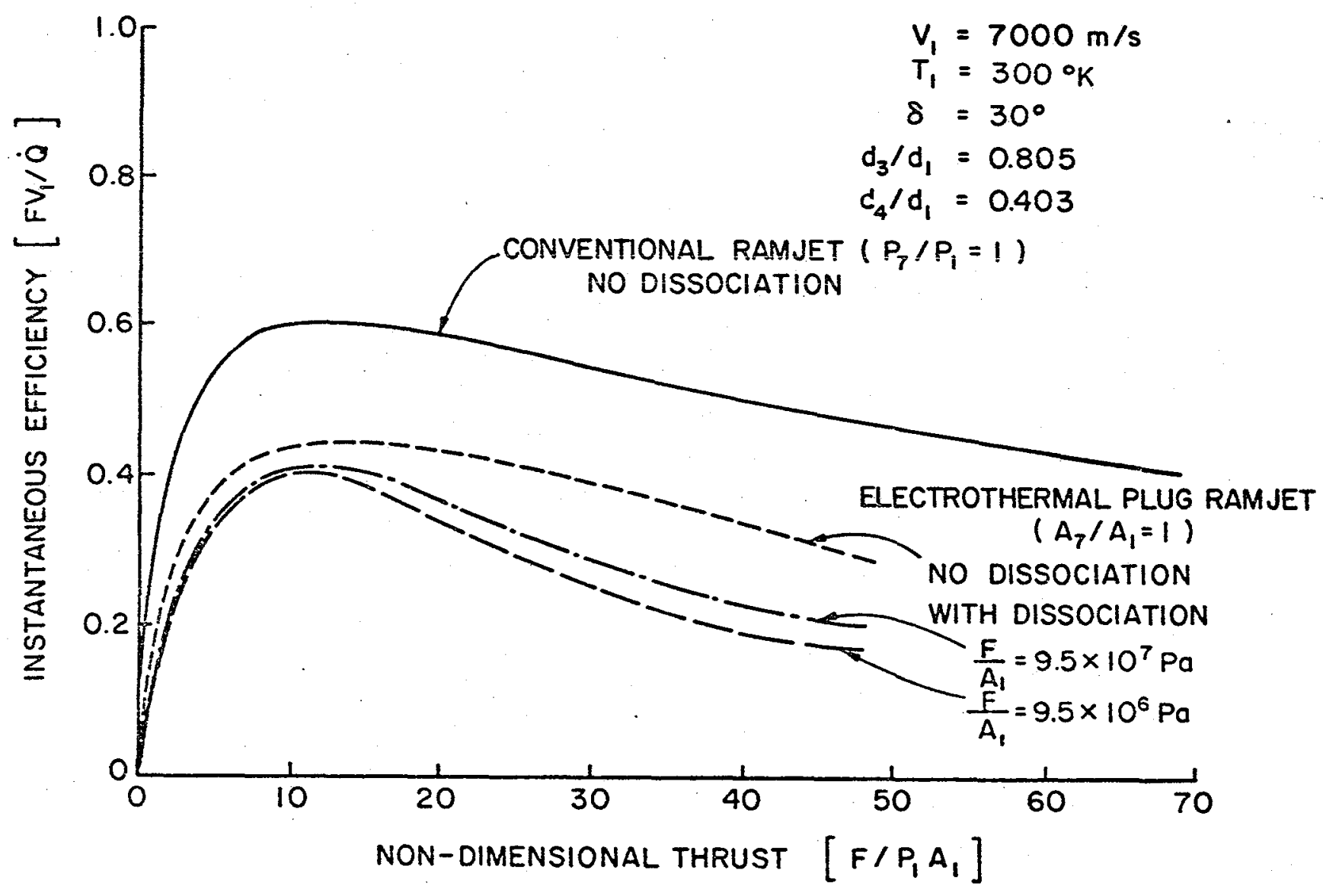

Fig. 4 Instantaneous Efficiency Profiles 
non-dimensional thrust for which each curve shown in Fig. 4 terminates is that value for which no more hea: could be added in the heat addition region of the respective rumjets without choking the flow at the exit of the heat addition region 5. All of the curves in Fig. 4 illustrate, that for a given velocity and diffuser geometry, there is a value for the non-dimensional thrust which provides the maximum instantaneous efficiency. It is also evident that, for the case of a dissociating propellant, a high tube pressure high thrust device corresponding to the higher thrusi: per unit area $\left(F / A_{1}=9.5 \times 10^{7} \mathrm{~Pa}\right)$ provides performance superior to a low tube pressure low thrust device $\left(F / A_{1}=9.5 \times 10^{6} \mathrm{~Pa}\right)$. This is due to the greater level of dissociation that occurs at the lower pressures. Figure 5 illustrates this by showing that the mass fraction of diatomic hydrogen in the ramjet exhaust is greater at the higher thrust per unit area level "here the initial tube pressure is higher at a given non-dimensional tlirust. It is evident that high values of the non-dimensional thrust can be achieved before dissociation becomes important and that increasing the initial tube pressure $P_{1}$ decreases the amount of dissociation in the propellant.

Figure 6 shows the effect of non-dimensional thrust on the fraction of the total thrust that is produced by the differential pressure-area product across the ramjet. The conventional ramjet operating with a perfect gas derives a smaller fraction of thrust from the pressure-area differential than the other ramjet configurations. The electrothermal plug ramjet operating with a perfect gas obtains a greater fraction of thrust from the differential of the pressure area product than the conventional ramjet for a given value of the non-dimensional thrust. The 
onisina pata

OF POOR Qu:?:

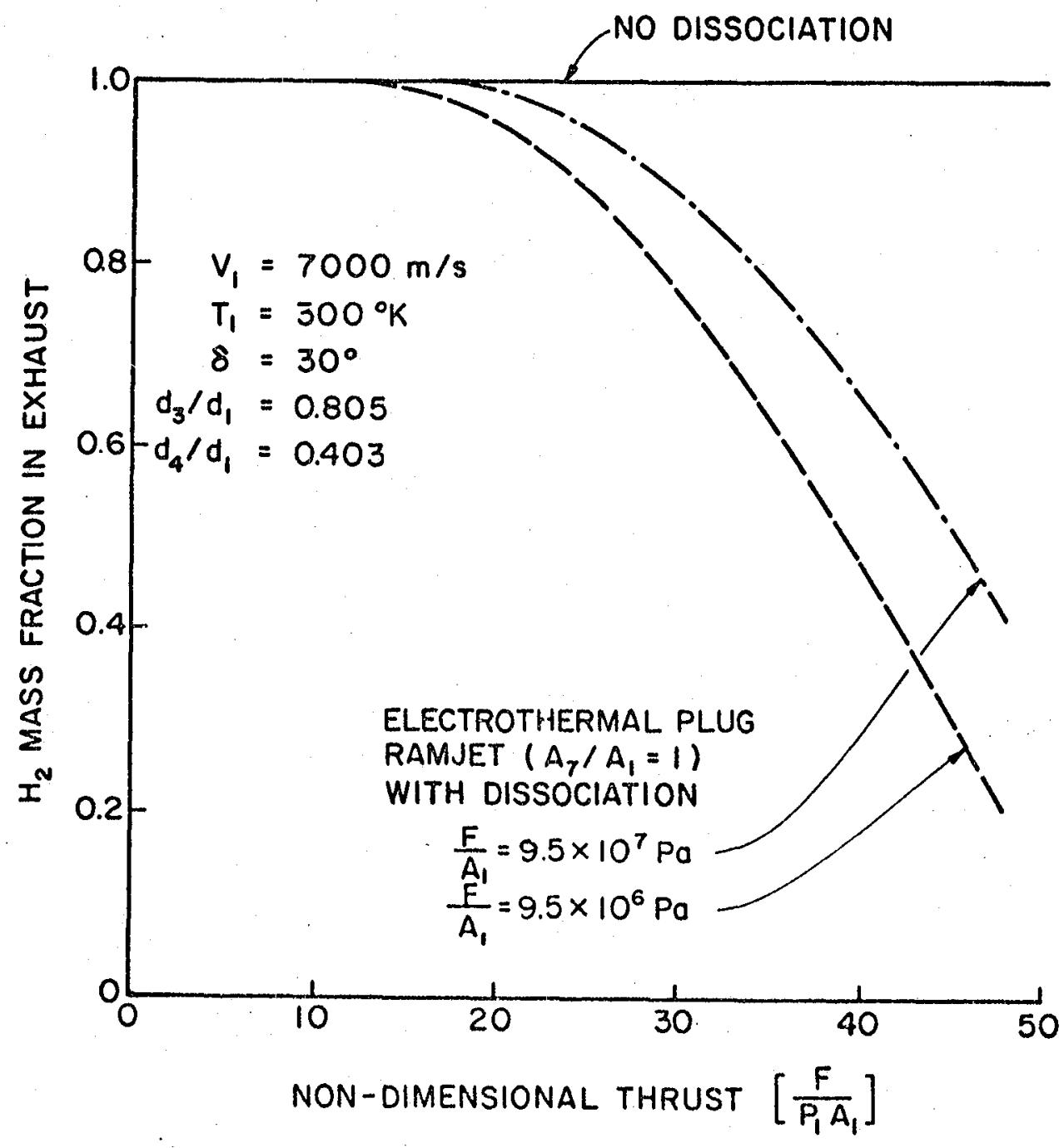

Fig. 5 llass Fraction of $\mathrm{H}_{2}$ in Exhaust 


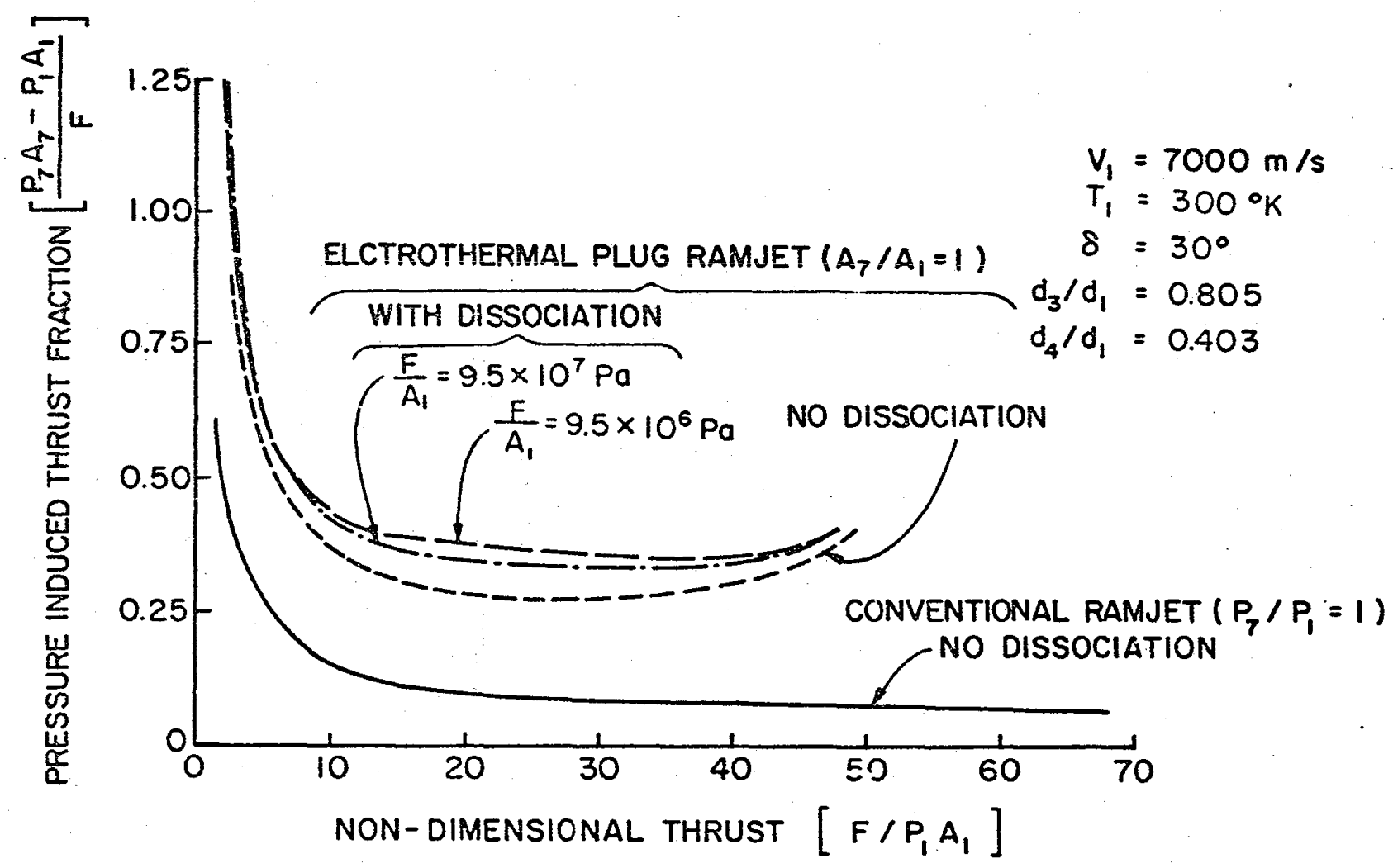

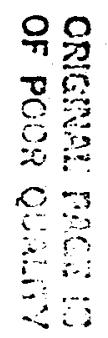

Fig. 6 Pressure-Induced Thrust Fraction Profiles 
electrothermal ramjet analyses which consider non-perfect gas effects show that including these effects causes the pressure induced thrust fraction to rise over that given for the perfect gas, electrothermal ramjet. The high tube pressure, high thrust ramjet operates with a lower pressure--induced thrust fraction than the low tube pressure, low thrust ramjet.

Figure 7 illustrates the relationship between the exit/inlet static pressure ratio and the non-dimensional thrust for the different ramjet models considered. By design, the exit/inlet pressure ratio is unity for the conventional ramjet. The electrothermal plug ramjet operating with a perfect gas exhibits lower pressure ratios than the electrothermal plug ramjet operating with a reacting propellant. For the electrothermal plug ramjet operating with a reacting propellant, high thrust, high pressure operation is seen to exhibit slightly lower exit/inlet pressure ratios than low thrust, low pressure operation.

The exit/inlet velocity ratios for the different ramjet configurations are shown in Fig. 8. The conventional ramjet exhibits a nearly linear dependence between the exit/inlet velocity ratio and the nondimensional thrust. It has been suggested previously that, for a conventional ramjet, both the thrust (Eq. 9) and the exit/inlet velocity ratio (Eq. 8) show rates of change approximately proportional to the square root of the total temperature ratio $\left(T_{T_{7}} / T_{T_{1}}\right)$, so a linear dependency between the thrust and the exit/inlet velocity ratio is to be expected. The results obtained for the electrothermal plug ramjet operating with a perfect gas show that the velocity ratio is degraded with respect to the conventional ramjet. This curve is not linear because the outlet/inlet static pressure ratio increases with 


\section{ELECTROTHERMAL PLUG RAMJET $\left(A_{7} / A_{1}=1\right)$}

WITH DISSOCIATION

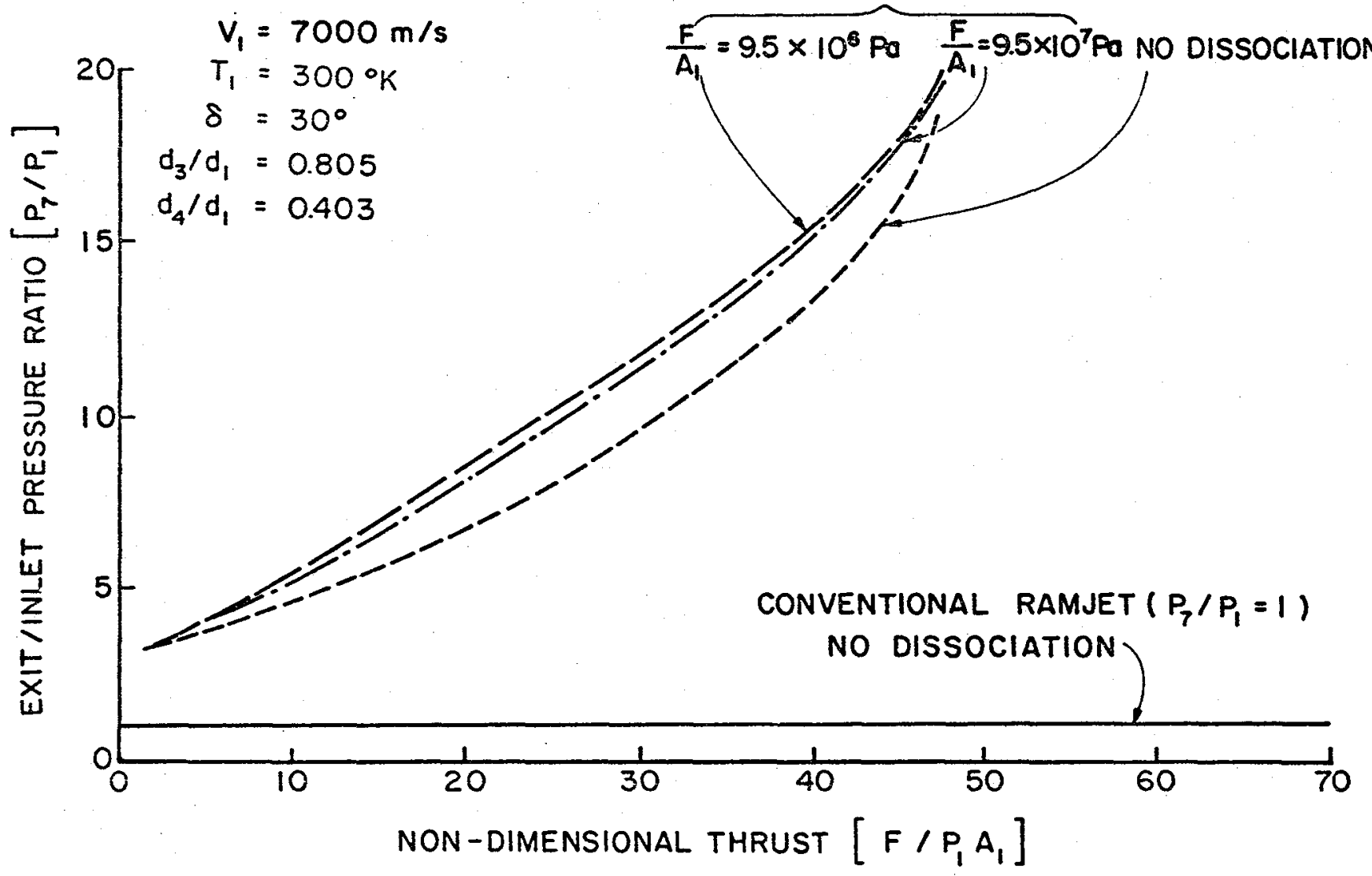

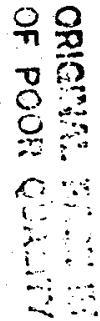

Fig. 7 Cutlet/Inlet Pressure Ratio Profiles 


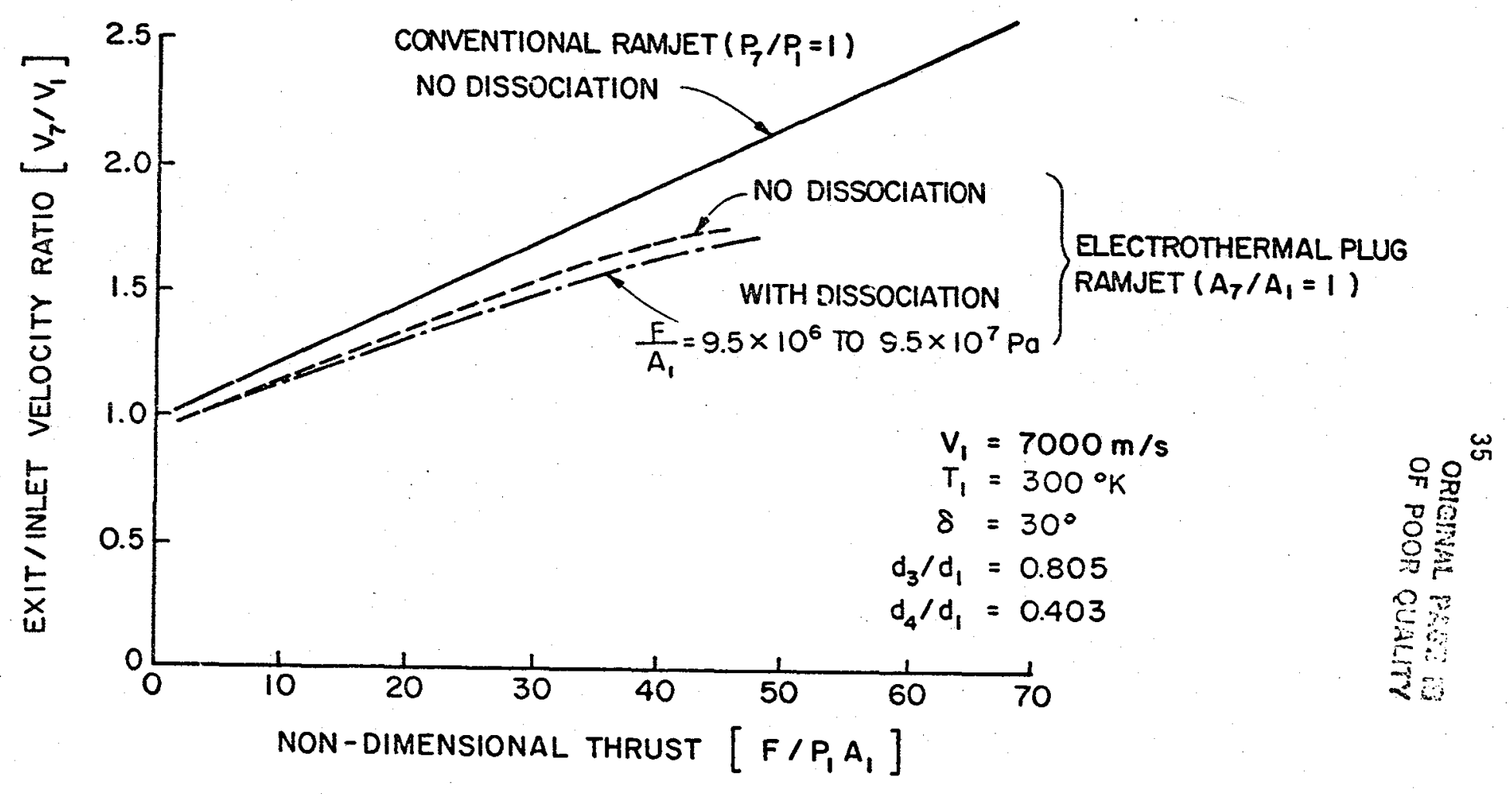

Fig. 8 Outlet/Inlet Velocity Ratio Profiles 


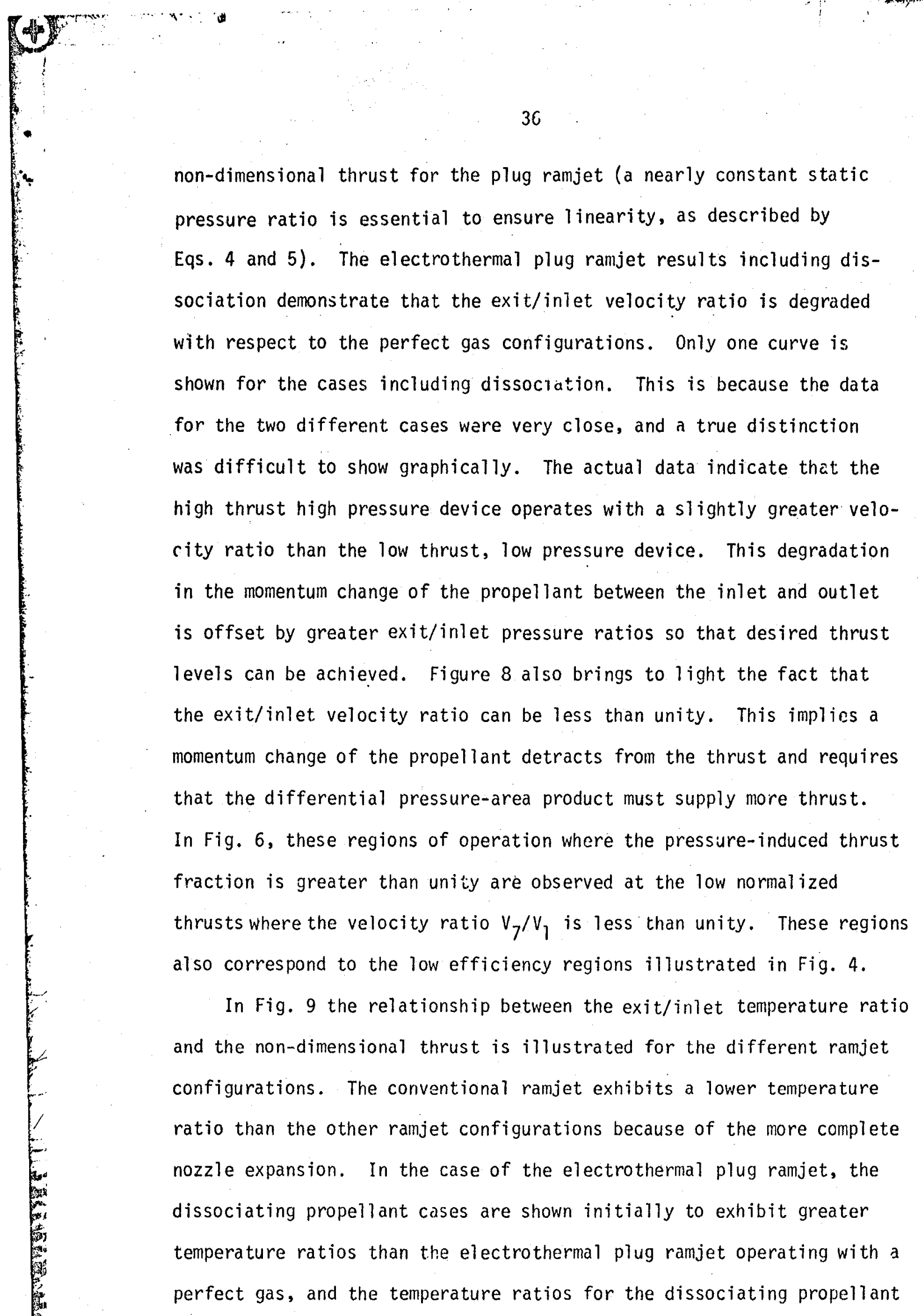




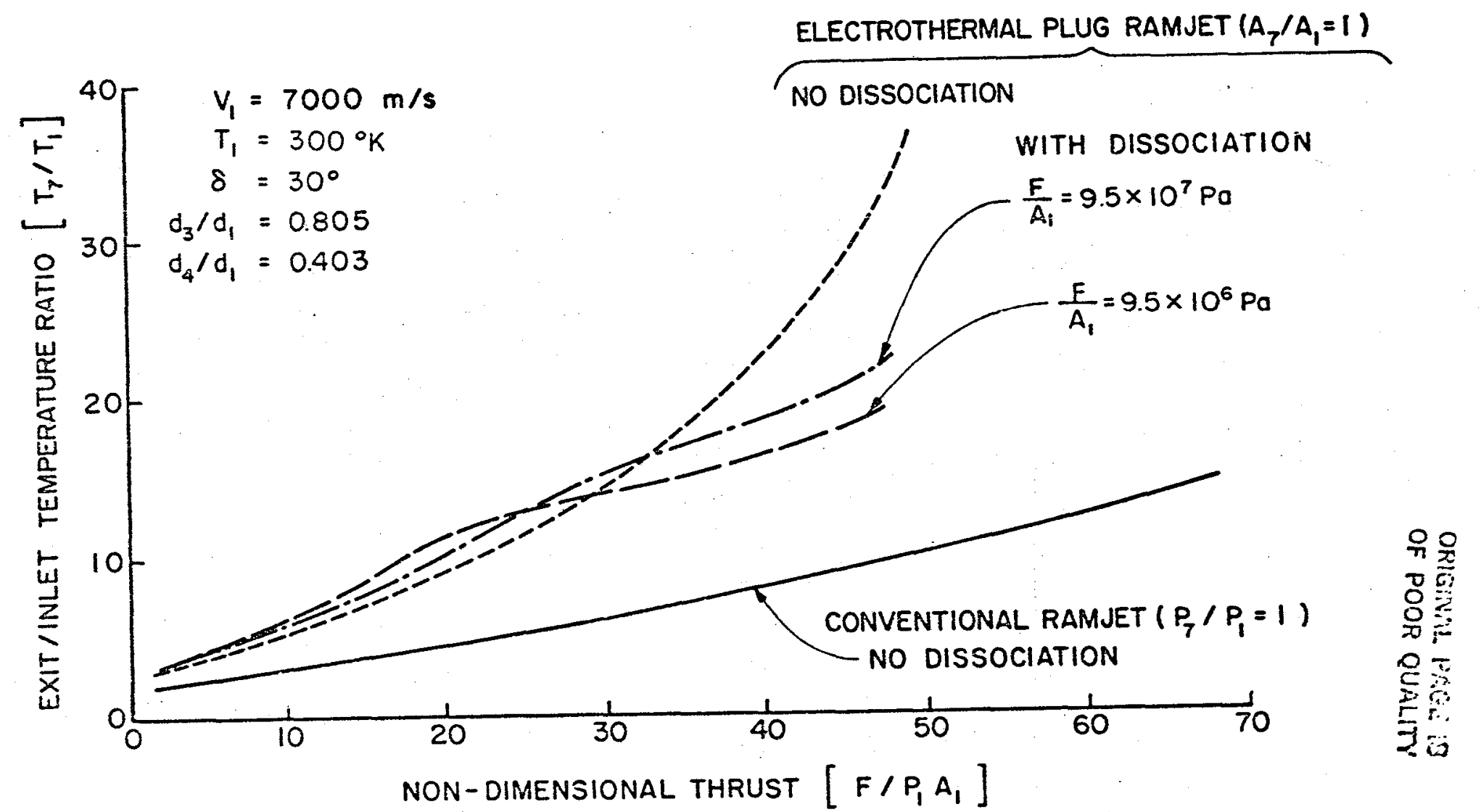

Fig. 9 Outlet/Inlet Static Temperature Profiles 
drop below those shown for the perfect gas electrothermal plug ramjet as the non-dimensional thrust is increased. This lowering of the exit/ inlet temperature ratio is due to the dissociation of the propellant.

The effect of changes in the flight velocity on the instantaneous efficiency can be seen by comparing the results of Fig. $4\left(V_{1}=7,000 \mathrm{~m} / \mathrm{s}\right)$ with those of Figs. $10\left(V_{1}=15,000 \mathrm{~m} / \mathrm{sec}\right)$ and Fig. $11\left(V_{1}=4,000 \mathrm{~m} / \mathrm{sec}\right)$. All of the other operational parameters mentioned previously, such as the diffuser cone half-angle, are the same as for the $7,000 \mathrm{~m} / \mathrm{s}$ flight velocity, except for the diameter ratios $d_{3} / d_{1}$ and $d_{4} / d_{1}$. The diameter ratio $d_{3} / d_{4}=2$ has been specified to be constant for any velocity. As the ramjet flight velocity increases, the conical shock angle will decrease if the conical diffuser half-angle is held constant. Because it has previously been specified that the intersection of the oblique shock and the tube wall occurs at the diffuser shoulder, the tube diameter must decrease as the projectile velocity increases for a constant ramjet annular diffuser geometry. This is why the diameter ratios $d_{2} / d_{1}$ and $d_{4} / d_{1}$ increase as the flight velocity increases. Examining Figs. 10 and 11 one sees that the same trends associated with the $7000 \mathrm{~m} / \mathrm{s}$ results are evident in these figures (i.e., there is a value of the non-dimensional thrust for which the instantaneous afficiency is optimized). A very important feature of ramjet operation is brought out by examining Figs. 4, 10 and 11. This feature is that in order for ramjet operation to remain at a reasonable efficiency level during acceleration, the non-dimensional thrust must vary greatly over the region of acceleration. Assuming that a constant thrust level $(F)$ is desired and that the inlet area $\left(A_{1}\right)$ does not change appreciably during the launch of a given plug projectile makes it apparent that the static 


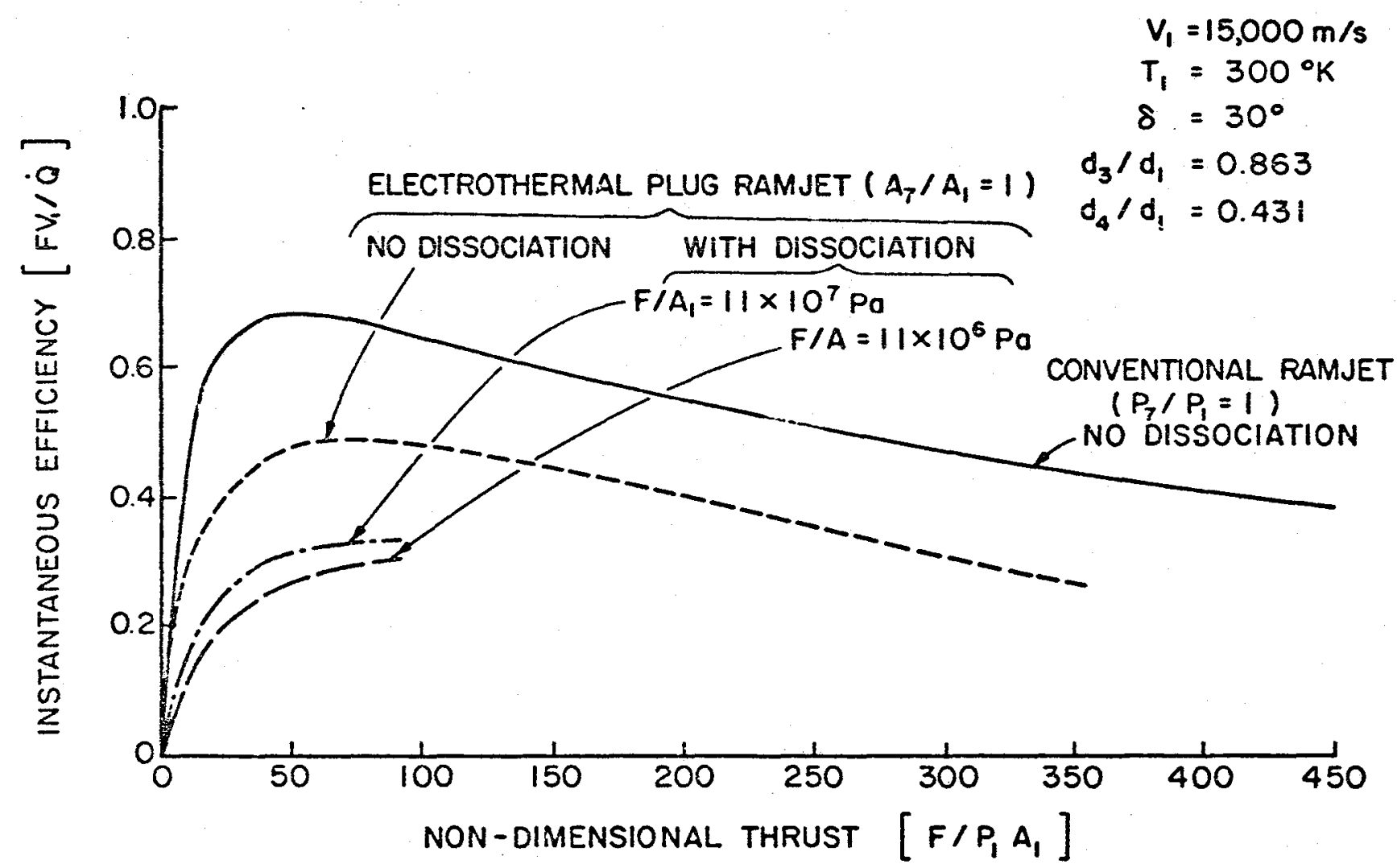

Fig. 10 Instantaneous Efficiency Profiles at High Velocity 


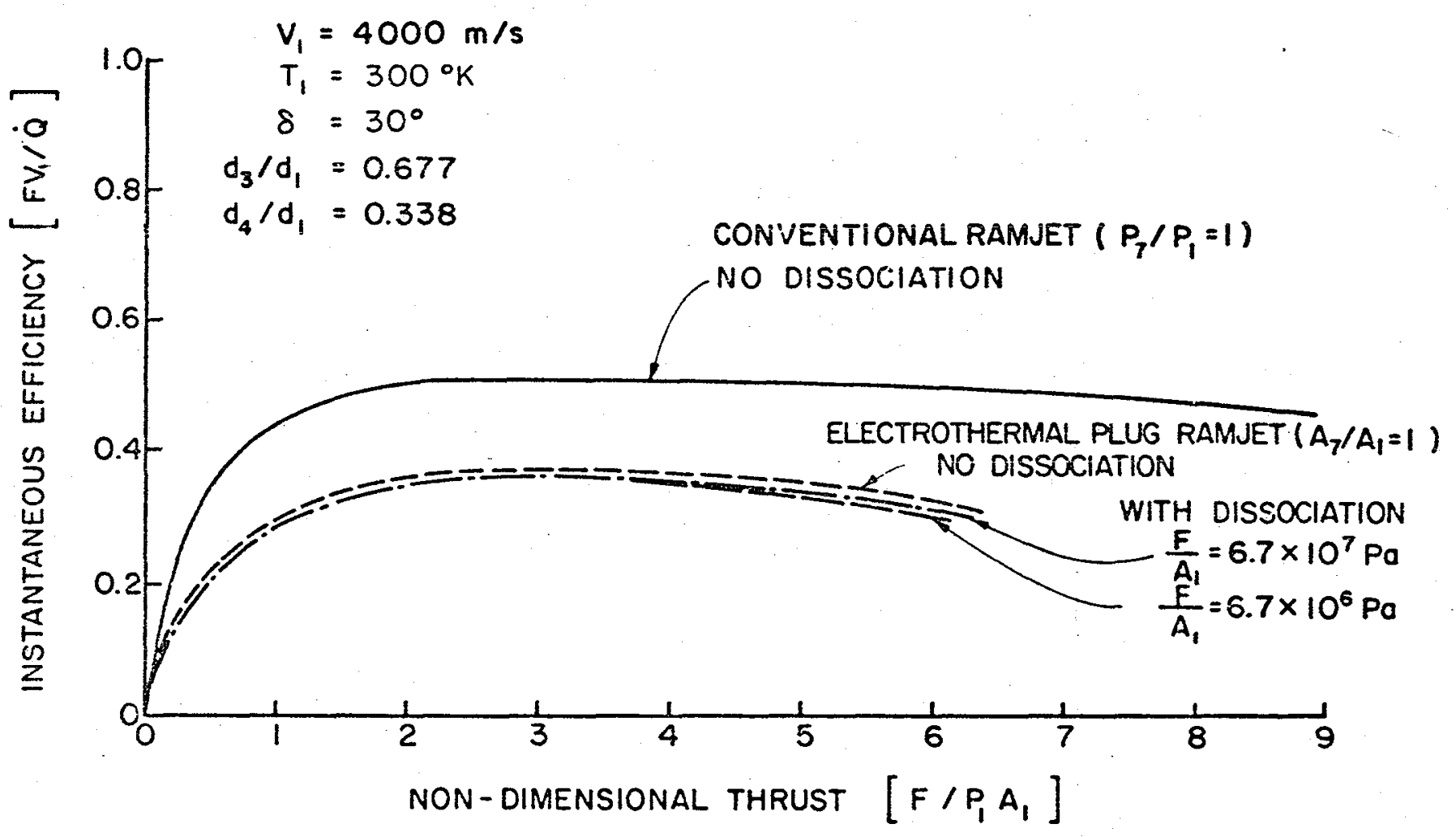

Fig. 11 Instantaneous Efficiency Profiles at Low Velocity 
propellant pressure imnediately upstream of the ramjet must decrease as the ramjet velocity increases so that efficient operation can be maintained. It is noted that the data in Fig. 10 for the dissociating propellant, electrothermal plug ramjet are terminated at a value of 90 for the nuni-úimensional thrust because ionization of the propellant started to become significant past this value.

The results of Fig. 12 were obtained from a series of plots like those in Figs. 4, 10 and 11 . They illustrate the variation in the nondimensional thrust which must be achieved to attain the maximum instantaneous efficiency over a velocity range of $4,000 \mathrm{~m} / \mathrm{s}$ to $15,000 \mathrm{~m} / \mathrm{s}$. The non-dimensional thrust is observed to vary from a minimum of 4 at $4,000 \mathrm{~m} / \mathrm{s}$ to a maximum of around 50 for a velocity of $15,000 \mathrm{~m} / \mathrm{s}$. These results are for the dissociating propellant.

Figure 13 illustrates the maximum instantaneous efficiency attainable for a mission where a projectile is accelerated from $4,000 \mathrm{~m} / \mathrm{s}$ to $15,000 \mathrm{~m} / \mathrm{s}$ utilizing two different thrust levels. The operational parameters are the same as previously defined. To attain the performance shown in Figure 13 the non-dimensional thrust must follow the nondimensionalized thrust profile given in Fig. 12. This means that $P_{1}$ must be varied continuously along the launch tube. For high velocities, the high thrust profile exhibits performance superior to the low thrust profile because high static tube pressures are associated with high thrust operation, and propellant dissociation is less significant for the high pressure case than for the low pressure case. The optimum value for the instantaneous efficiency is observed to be rear $7,000 \mathrm{~m} / \mathrm{s}$ because this is the velocity for which this particular diffuser 


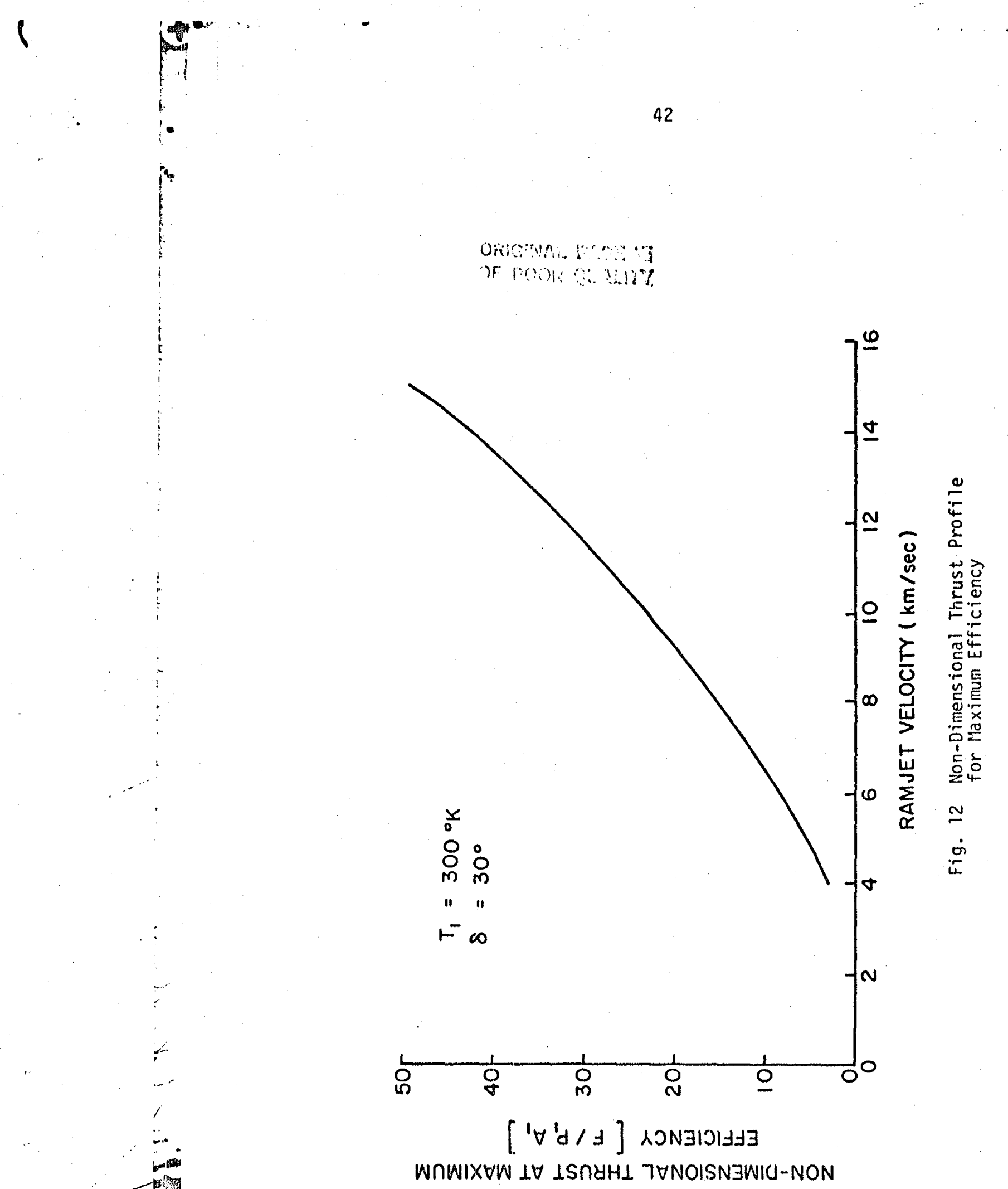




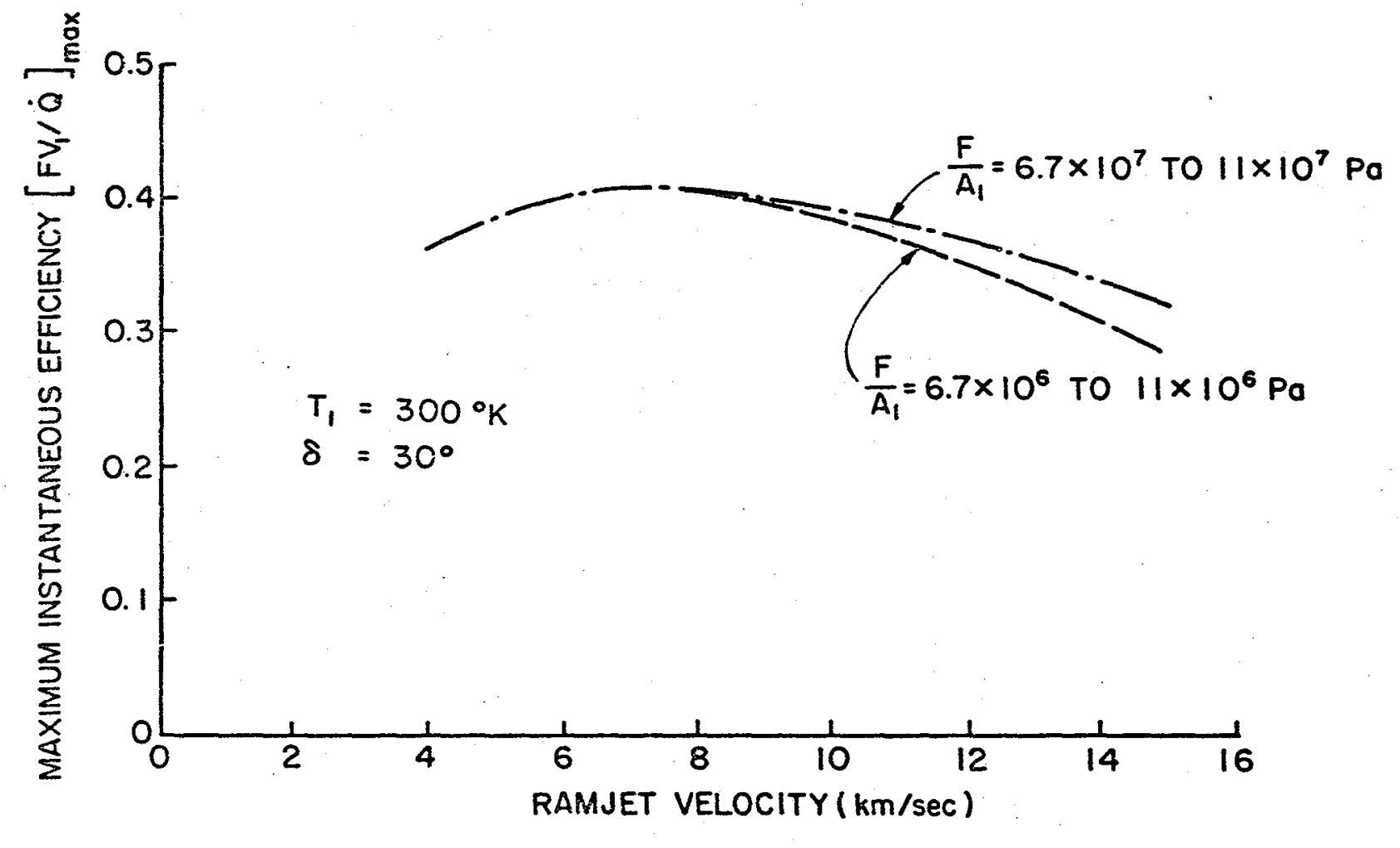

Fig. 13 llaximum Instantaneous Efficiency Profiles

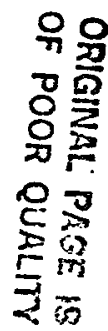


performs the best. Changing the diffuser geometry will change the location of the optimum point.

Several profiles are shown in Fig. 14 which detail the performance of a ramjet as it accelerates $10 \mathrm{~kg}$ at a level of $30,000 \mathrm{~g}$ 's. These profiles were computed for virious conical diffuser half-angles and for tube static pressures that remain at fixed values along the tube length. For purposes of comparison, the optimum efficiency performance data for the high thrust per unit area values given earlier are plotted with the results obtained for constant tube pressure acceleration. These values of the thrust per unit area coupled with the specified projectile mass produces an acceleration of $30,000 \mathrm{~g}$ 's. The early portions of the curves where no data are shown are those portions of the launches where the ramjet propulsion system could not achieve the specified thrust and where light gas gun induced acceleration would be used. The influence of the tube pressure can be clearly seen by comparing the results for tube pressures $\left(P_{1}\right)$ of 30 and 60 atmospheres and for a conical half-angle of $30^{\circ}$. Early in the operation, a high tube pressure is shown to exhibit superior performance, while towards the end of the acceleration period the lower tube pressure exhibits the superior performance. The resuits plotted for the optimum performance sugges that the variation in tube pressure is important in order to maintain high efficiency.

The effect of varying the conical diffuser half-angle is also illustrated in Fig. 14. Having a diffuser cone half-angle that is either too shallow or too steep degrades the performance. For the missions analyzed in this paper, the overall optimum conical half-angle was near $30^{\circ}$. 


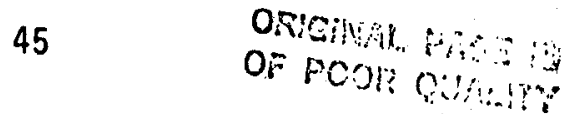

$\nabla \delta=30^{\circ}$ P VARIED FOR OPTIMUM PERFORMANCE

$0 \delta=30^{\circ} \quad P_{1}=60 \mathrm{orm}$

ㄷ $\delta=45^{\circ} \quad P_{1}=30 \mathrm{otm}$

$\triangle 8=10^{\circ} \quad P_{1}=30$ atm

$\diamond \delta=30^{\circ} \quad P_{1}=30 \mathrm{~atm}$
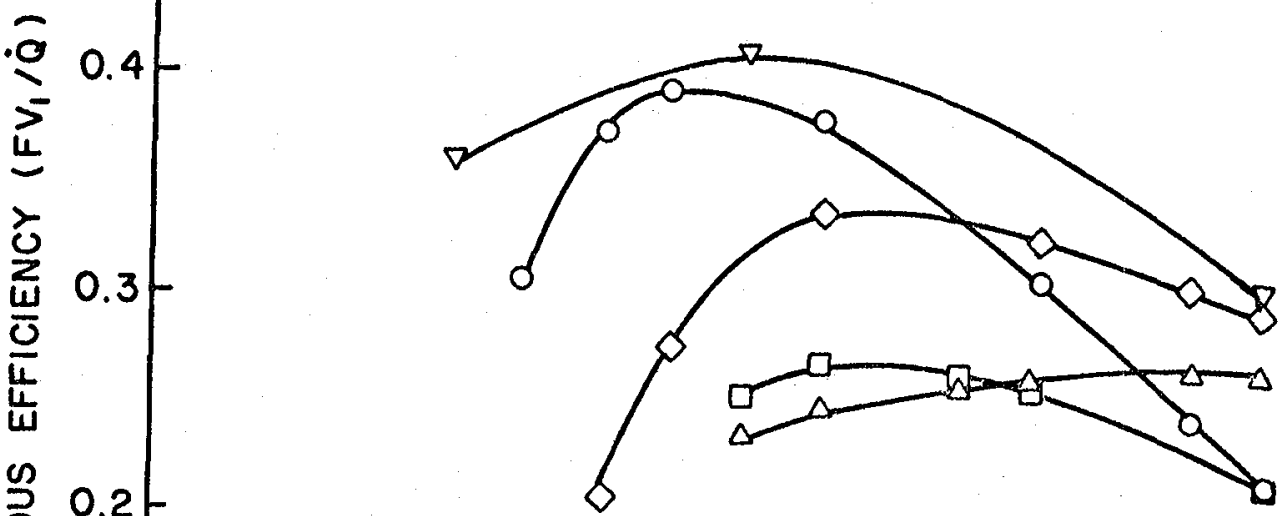

$\sigma=30,000 \mathrm{~g}^{\prime} \mathrm{s}$

$T_{1}=300^{\circ} \mathrm{K}$

$0.1-$ 
By applying the first law of thermodynamics and the momentum equation to the control volume shown in Fig. $3 \mathrm{~b}$, expressions for the non-dimensional thrust and the instantaneous efficiency can be put in the following forms respectively

$$
\begin{aligned}
& \frac{F}{P_{1} A_{1}}=\frac{\rho_{1} V_{1}^{2}\left(V_{7} / V_{1}-1\right)+P_{1}\left(P_{7} / P_{1}-1\right)}{P_{1}} \\
& \frac{F V_{1}}{\dot{Q}}=\frac{\rho_{1} V_{1}^{2}\left(V_{7} / V_{1}-1\right)+P_{1}\left(P_{7} / P_{1}-1\right)}{\rho_{1} q}
\end{aligned}
$$

Examination of Eqs. 12 and 13 illustrates that for given constant inlet conditions the non-dimensional thrust and the instantaneous efficiency will remain constant if the heat addition per unit mass $(q)$, the exit/ inlet velocity ratio $\left(V_{7} / V_{1}\right)$ and the exit/inlet pressure ratio $\left(P_{7} / P_{1}\right)$ remain constant. In order for these parameters to remain constant it is required that the propellant thermodynamic properties remain constant throughout the ramjet flowfield shown in Fig. $3 b$. To ensure a consistent solution for the flowfield at state 4 shown in Fig. $3 b$, it is necessary to keep the diameter ratio $d_{1} / d_{4}$ and the conical diffuser half-angle $(\delta)$ constant for whatever flight velocity is being considered. Keeping these variables constant allows the results given here to be scaled for different size projectiles and thrust levels. 


\section{CONCLUSIONS}

The results presented in this thesis have extended the results obtained from earlier analyses. 1 The earlier analyses assumed isentropic diffuser operation and perfect gas propellant behavior, while the present analysis takes into account such effects as diffuser shock losses, dissociation and ionization of hydrogen propellant, and nonequilibrium processes in the supersonic nozzle expansion. It has been demonstrated that diffuser losses, while significant, do not degrade ramjet performance to unacceptably low levels. The diffuser geometry does affect performance significantly if the diffuser conical halfangle is too shallow or too steep. At the overall optimum conical half-angle for the cases presented here $\left(\sim 30^{\circ}\right)$ however the performance degradation is not excessive.

In the analyses conducted in this thesis, it has been assumed that the propellant is in thermodynamic equilibrium up to the throat of the annular nozzle, and nonequilibrium processes are allowed to occur in the supersonic nozzle expansion downstream of the nozzle throat. Chemical nonequilibrium in the nozzle expansion was evaluated, and it was found that the nozzle flowfield was typically found to be very near chemical equilibrium. Because chemical relaxation times are generally larger than other nonequilibrium process relaxation times (such as vibrational, translational and rotational processes) the assumption that the propellant is in thermodynamic equilibrium should not introduce significant errors into the analyses. 
It has been demonstrated that the static propellant pressure immediately upstream of the electrothermal ramjet affects performance significantly. Changing this pressure effectively changes the mass flow rate and the rate of heat addition per unit mass and it is demonstrated that for a particular flight velocity there is an optimum rate of heat addition per unit mass. When dissociation and ionization are allowed to occur, it is shown that decreasing the static propellant pressure increases the losses due to dissociation and ionization. However, the results presented demonstrate that it is feasible to operate in operational regimes where dissociation and ionization do not significantly degrade ramjet performance. To maintain peak efficiency the static propellant pressure immediately ahead of the ramjet must decrease as ramjet velocity increases.

The assumption of quasi-steady fluid flow used in this analysis will surely introduce some error. It is possible that the mass of the propellant contained within the ramjet could change, and the fact that the propellant within the ramjet is also accelerating could also introduce significant errors. The acceleration could cause a "hydrostatic" pressure difference to occur within the ramjet heat addition zone, which would possibly invalidate the results presented. An initial analysis of these effects has been conducted and is detailed in Appendix $r$. This analysis demonstrates that the non-steady gas dynamic effects just mentioned do not introduce errors which invalidate the results which are presented. The results from this analysis are based on calculations which compute flowfield changes over typical ramjet fluid particle residence times. Indeed, the idea is put forward that 
proper tailoring of the upstream propellant conditions will minimize the unsteady gas dynamics effects to the point where they are not important.

Much analysis remains to be done to investigate completely the effects of all of the parameters of concern. The problems of projectile material selection and design have not been addressed nor have the details of the heat addition process been examined. It is possible that pressure oscillations could occur in a device of this type, and these oscillations will be related to the unsteady gas dynamics effects. Additional work related to maintenance of the correct projectile base pressure and static pressure immediately ahead of the projectile remains to be done. Although this aspect of the problem has not yet been studied, it intuitively seems possible that methods will be found which will allow for maintenance of the correct pressures. It is also possible that three-dimensional flow effects could become significant.

The results presented earlier have demonstrated that the conventional ramjet has the capability to outperform the plug ramjet. However, the mechanical complexities associated with conventional ramjet operation (such as providing efficient shock swallowing and producing the necessary nozzle conditions) are very significant.. It is therefore argued that the plug ramjet, because of its apparent mechanical simplicity, is a more desirable device for effecting high acceleration launches.

The results presented suggest that the electrothermal ramjet concept cannot be rejected on the basis that it violates a basic principle of fluid mechanics or thermodynamics. Further, the results presented suggest that the electrothermal ramjet thas potential of accelerating payloads to high velocity with a good energy utilization 


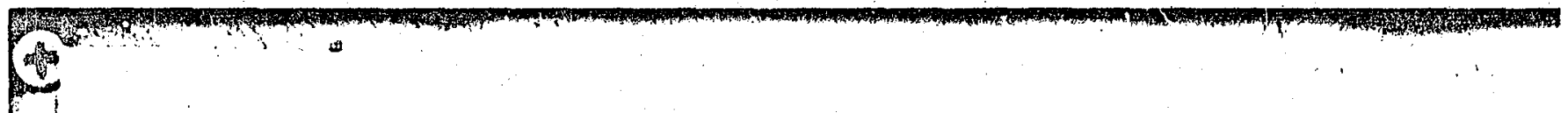

efficiency. For these reasons it is concluded that further analys is related to the design and development of the electrothermal ramjet concept is warranted. 


\section{REFERENCES}

1. Wilbur, P. J., Mitchell, C. E. and Shaw, B. D., "The Electrothermal Ramjet," Journal of Spacecraft and Rockets, November-December 1983, fp. 603-610.

2. Shaw, B. D., "The Electrothermal Ramjet," appears in "Advanced Ion Thruster and Electrothermal Launcher Research," P. J. Wilbur ed., NASA-CR-168083, Jan. 1983, pp. 36-48.

3. Mitchell, C. E., "Constant Pressure Acceleration of the Electrothermal Ramjet," appears in "Advanced Ion Thruster and Electrothermal Launcher Research," P. J. Wilbur ed., NASA-CR168083, Jan. 1983, pp. 49-71.

4. Lukasiewicz, J., Experimental Methods of Hypersonics, Marcel Dekker, Inc., 1973, pp. 95-101.

A7. Anderson, J. D., Modern Compressible Flow, McGraw-Hill, New York, 1982, Chap. 10.

A2. Lukasiewicz, J., Experimental Methods of Hypersonics, Marcel Dekker, Inc., 1973, pp. 95-101.

B1. Bond, J. W., Watson, K. M., and Welch, J. A., Atomic Theory of Gas Dynamics, Addison-Wes ley, Reading, MA, 1965, p. 85.

B2. Bond, J. W., Watson, K. M., and Welch, J. A., Atomic Theory of Gas Dynamics, Addison-Wesley, Reading, MA, 1965, p. 100.

B3. Bond, J. W., Watson, K. M., and Welch, J. A., Atomic Theory of Gas Dynamics, Addison-Wesley, Reading, MA, 1965, p. 86.

B4. Zel'dovich, Y. B., and Raizer, Y. P., Physics of Shock Waves and High Temperature Hydrodynamic Phenomena, Volume 1, Academic

B5. Svehla, R. A., "Thermodynamic and Transport Properties for the Hydrogen-0xygen System," NASA SP-3011, 1964.

Cl. Vincenti, W. G. and kruger, C. H., Introduction to Physical Gas Dynamics, John Wiley and Sons, New York, 1965, p. 298. 
APPENDIX A

CONICAL FLOWFIELD AVERAGING 
APPENDIX A NOMENCLATURE

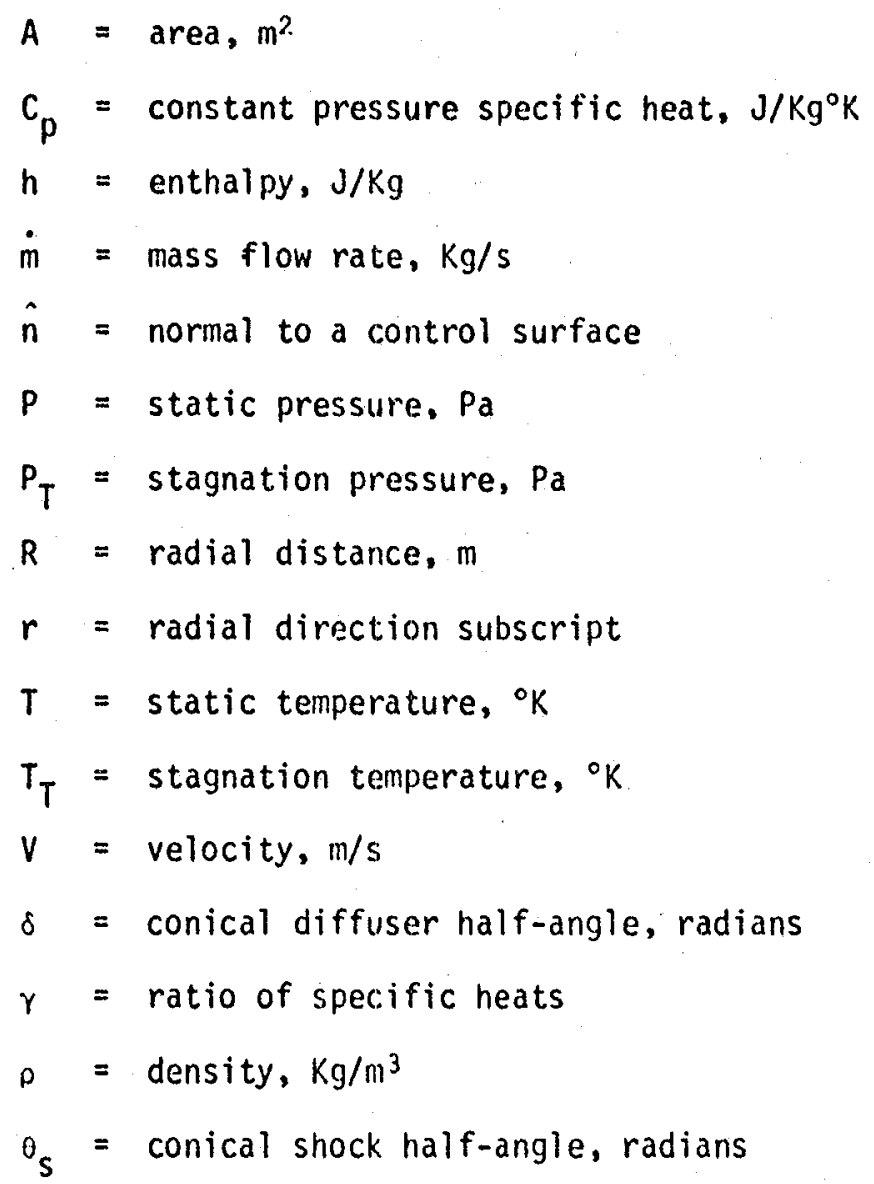
average value, a numerical subscript denotes a position shown in Figure $A l$, and a subscript of $r$ or $\theta$ indicates a direction as specified in Figure A2. 


\section{APPENDIX A}

\section{CONICAL FLOWFIELD AVERAGING}

In this analysis it is assumed that the working fluid behaves as a perfect gas, and that the flowfield is axis-symmetric and quasi-steady. Assuming that the working fluid behaves as a perfect gas should not introduce too much error because for shallow cone angles the flowfield conditions should not change significantly across a conical shoik even for high incoming Mach numbers. The quasi-steady assumption is justified in Appendix $D$ where it is shown that fluid particle residence times are small compared to the times required for flowfield changes to be significant. The geometric constraints should not let the ramjet projectile deviate significantly from axis-symmetric conditions, so assuming axis-symmetric flowfields exist should not introduce significant errors.

Consider a conical diffuser of the type shown in Fig. Al to be operating at a zero angle of attack within a launch tube. Assume that. the projectile is traveling at a high enough velocity so that conical shock attachment is assured. The conical shock formed will intersect the tube wall as shown in Figure Al. For a given conical half-angle $(\delta)$ che flow from $i$ to 2 can be calculated given conditions at 1 using the Taylor-Maccol method. ${ }^{A l}$ Indeed, the conditions anywhere within the axis-symmetric flowfield can be calculated using this method. The problem is to describe the flow between 1 and 4 for given conaitions at 1 and a specified diffuser geometry. In reality a pattern of reflected oblique shock waves would exist downstream of the initial conical shock depending on the annular diffuser throat area and the 


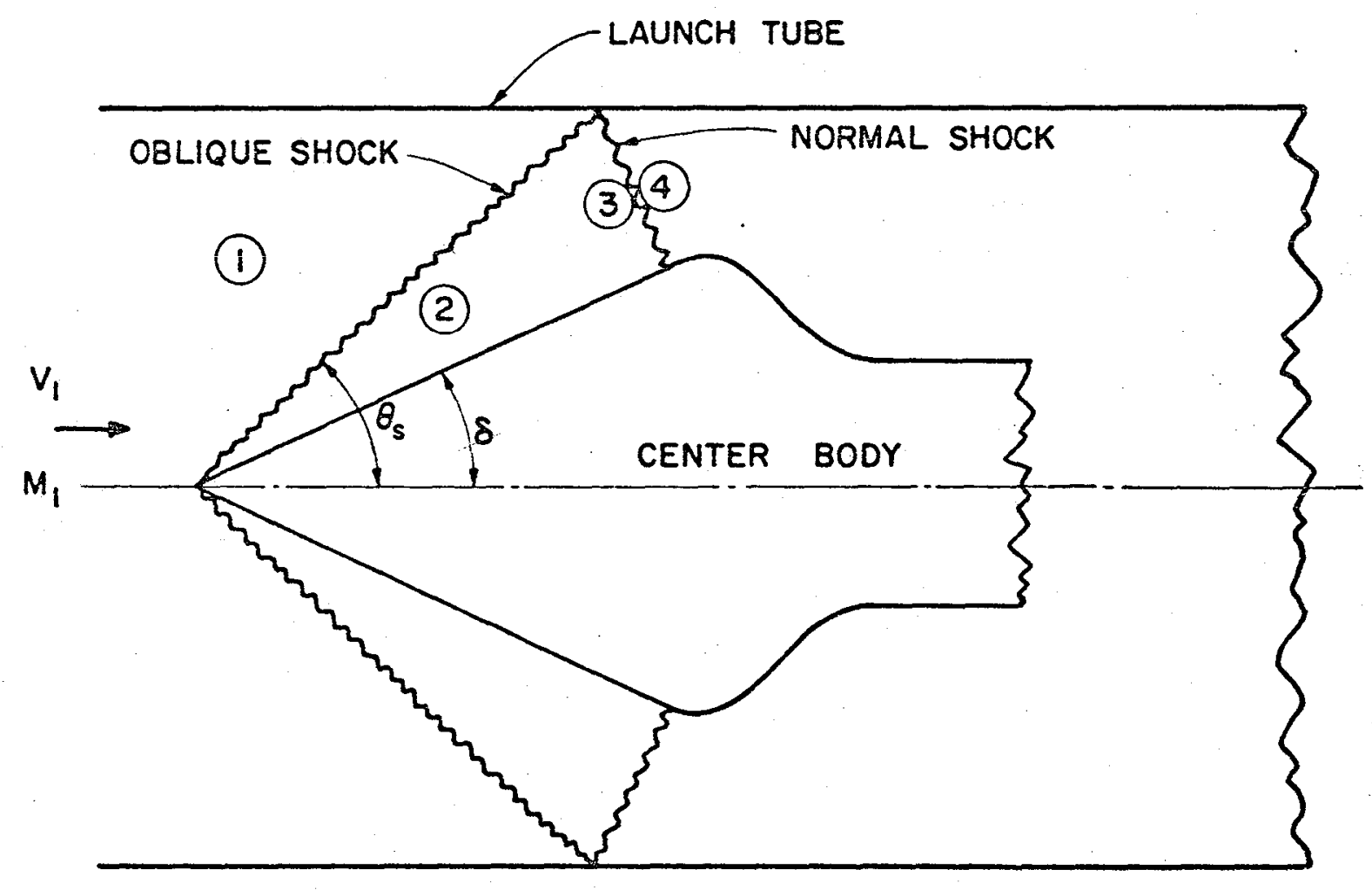

Fig. Al Electrothermā Ramjet Diffuser 
pressure at 4 . If it happens that the flow is restricted at the diffuser throat or downstream of 4 then a bow shock could exist ahead of the diffuser. If the downstream conditions are selected as they are for designs considered here so that the flow is not restricted and that the back pressure conditions are correct then a reflected shock pattern terminated by a normal shock will exist. The location of the terminating normal shock can be dictated by varying the diffuser throat area and the pressure at 4.

For hypersonic flows, because of separation and boundary layershock wave interaction effects, diffusers perform close to what is predicted for normal shock diffusers. ${ }^{A 2}$ Thus in this analysis the following assumptions are used:

1) The separation and boundary layer-shock interaction behavior does not become important until the refiected shock pattern begins downstream of the intersection of the tube wall with the initial conical shock. Neglecting separation and boundary layer effects in this initial conical flowfield region should not produce large errors because the scale of the diffuser is expected to be small. Neglecting these effects also allows the Taylor-Maccol method of analyzing conical flowfields to be utilized.

2) The flow downstream of the initial conical flowfield is rapidly mixed and can be modeled by a one-dimensional flow which satisfies the conservation laws. It is noted that the conservation laws are insufficient to completely specify the one-dimensional flow; an additional assumption which will be described shortly is also needed. 


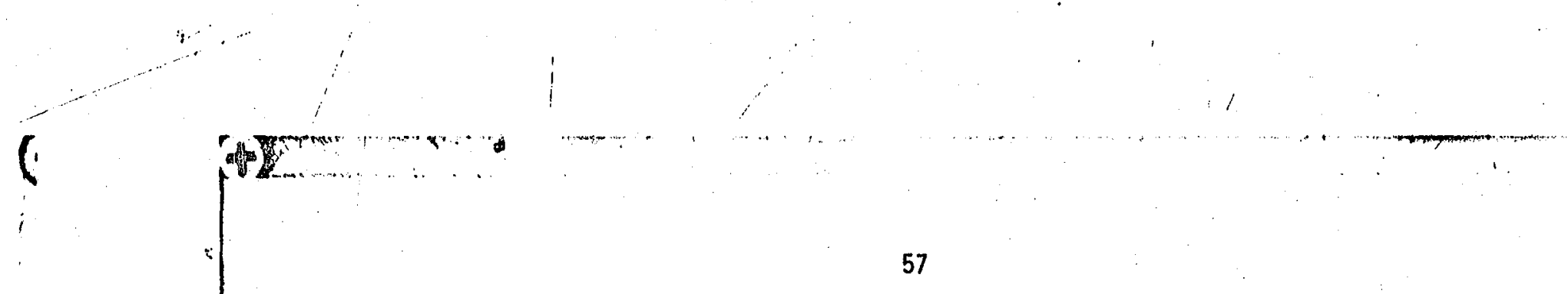

3) A normal shock terminates the one-dimensional supersonic flowfield.

When these assumptions are applied the flow processes in the diffuser can be modelled in the following way suggested by Fig. Al. The free stream flow passes from state 1 through the conical shock into the conical flowfield of region 2. The properties of this flowfield, which vary from point to point and must be calculated using a numerical procedure, are averaged over the surface immediately upstream of the normal shock to obtain the properties at 3 . These averaged properties serve as input to the one-dimensional normal shock relations and yield the properties of the fluid immediately downstream of the normal shock at state 4.

The additional statement needed to connect the conical and onedimensional flowfields will now be considered. The choice used here is made through the definition of a mass flow rate averaged velocity for the conical flowfield and equating this to a characteristic onedimensional velocity $\bar{V}_{3}$. In Fig. Al let states 2 and 3 represent the conical and one-dimensional flowfield states respectively. The mass flow rate average velocity at 3 is defined by

$$
\nabla_{3}=\frac{\int_{c . s}|\vec{V}| \rho(\vec{V} \cdot \hat{n}) d A}{\text { c.s } \int(\vec{v} \cdot \hat{n}) d A}
$$

where the subscript c.s. refers to any arbitrary control surface which the complete conical flowfield passes through. The integration is simplified if a spherical coordinate system is placed at the vertex of the physical cone and the control surface is taken to be a spherical cap 
as is shown in Figure A2.

For this choice

$$
\begin{aligned}
& \vec{v} \cdot \hat{n}=V_{r}=\text { velocity in the radial direction } \\
& |\vec{V}|=\left(V_{r}^{2}+V_{\theta}^{2}\right)^{\frac{1}{2}} \\
& \rho=\rho(\theta) \quad \text { [for conical flow] } \\
& d A=2 \pi R^{2} \sin \theta d \theta .
\end{aligned}
$$

Thus it follows that

$$
\bar{V}_{3}=\frac{\int_{\delta}^{\theta_{s}} \rho\left(V_{r}^{2}+V_{\theta}{ }^{2}\right)^{\frac{1}{2}} V_{r} 2 \pi R \sin \theta d \theta}{\int_{\delta}^{\theta_{s}} \rho V_{r} 2 \pi R^{2} \sin \theta d \theta} .
$$

In this expression the factor $2 \pi R^{2}$ appears in both the numerator and denominator and thus will cancel. This implies that the one-dimensional properties of a conical flowfield are independent of the scale of the flow conditions. It follows then that the value for the average velocity immediately upstream of the normal shock is calculated from

$$
\vec{v}_{3}=\frac{\int_{\delta}^{\theta} s \rho v_{r}\left(v_{r}^{2}+v_{\theta}^{2}\right)^{\frac{1}{2}} \sin \theta d \theta}{\int_{\delta}^{\theta} \rho v_{r} \sin \theta d \theta} .
$$

Mass continuity, which must also be satisfied in the conical flowfield, requires that

$$
\bar{\rho}_{3} \bar{V}_{3} A_{3}=\int_{\delta}^{\theta} s \pi R^{2} \rho V_{r} \sin \theta d \theta=\dot{m}_{3}
$$




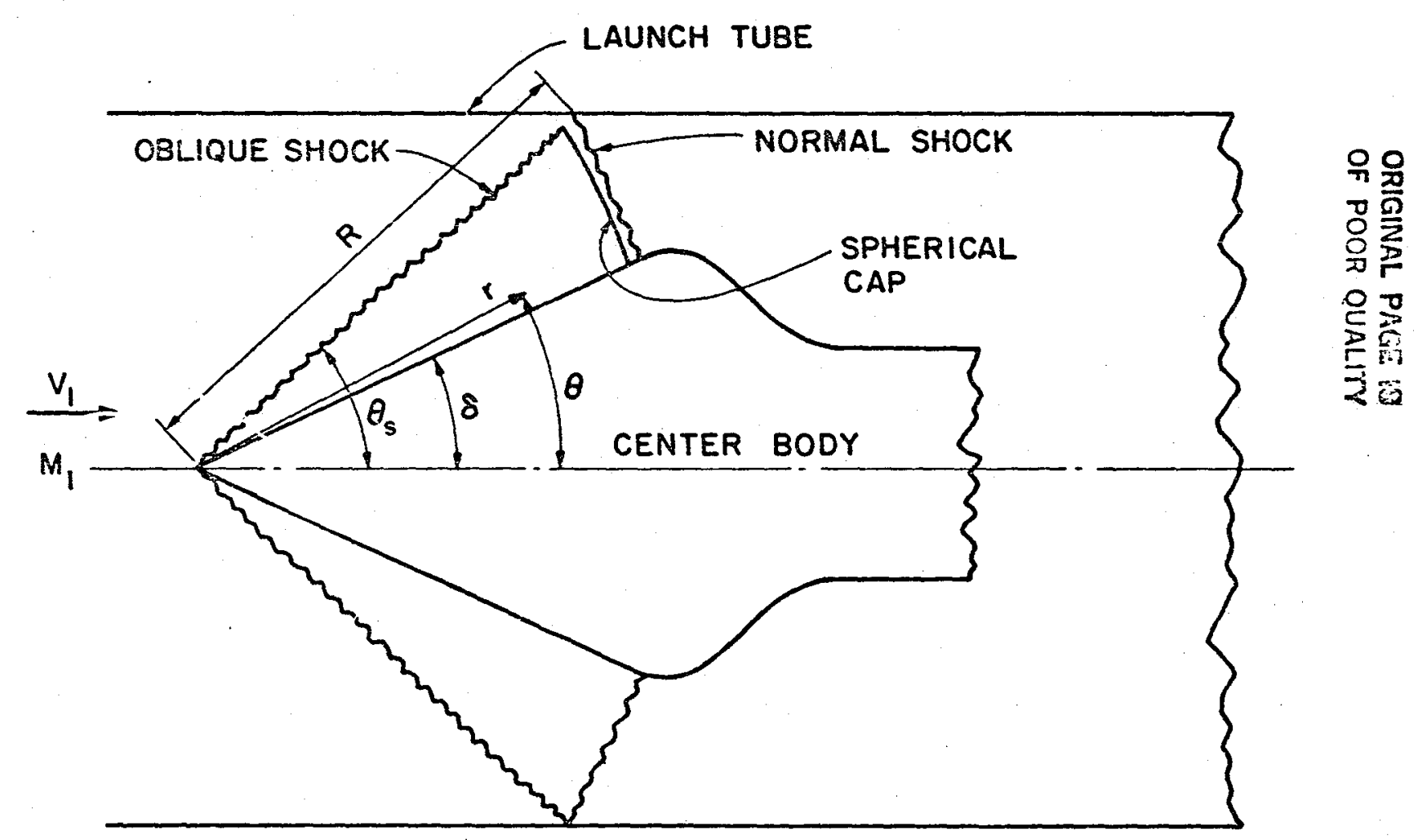

Fig. A2 Conical Flow Coordinate System 
where

$$
A_{3}=2 \pi R^{2} \int_{\delta}^{\theta} S \sin \theta d \theta=-2 \pi R^{2}\left(\cos \theta_{S}-\operatorname{Cos} \delta\right) .
$$

Combining these expressions one obtains an expression for the mean density immediately upstream of the normal shock.

$$
\overline{\tilde{r}}_{3}=\frac{\int_{\delta}^{\theta} s v_{r} \sin \theta d \theta}{\bar{V}_{3}\left(\cos \delta-\cos \theta_{s}\right)}
$$

The first law of thermodynamics must also be satisfied.

$$
n_{T_{3}}=n_{T_{1}}=C_{p} \bar{T}_{3}\left[1+\frac{\gamma-1}{2} M_{3}^{2}\right]
$$

Solving for the mean temperature upstream of the normal shock yields

$$
\bar{T}_{3}=\frac{h_{T_{1}}}{C_{p}}-\frac{\gamma-1}{\gamma} \frac{\bar{V}_{3}^{2}}{2 R}
$$

The ideal gas equation is assumed to be valid.

$$
\bar{P}_{3}=\bar{\rho}_{3} R \bar{T}_{3}
$$

The average stagnation pressure is defined by

$$
\overline{\mathrm{P}}_{\mathrm{T}_{3}}=\overline{\mathrm{P}}_{3}\left(\frac{\mathrm{T}_{\mathrm{T}_{3}}}{\overline{\mathrm{T}_{3}}}\right)^{\frac{\gamma}{\gamma-T}} \text {. }
$$

These average values for the flowfield variables are used as input for the normal shock relations.

The only criterion applied to determine the annular diffuser throat area is that this area be large enough to swallow the flow downstream of the normal shock. 
The Taylor-Maccol method of analyzing conical flowfields, which was utilized in the analyses conducted in this thesis, is not presented. For a complete description of this procedure see reference Al. 
APPENDIX B

\section{EQ̨UILIBRIUM HYDROGEN PROPERTIES}


APPENDIX B NOMENCLATURE

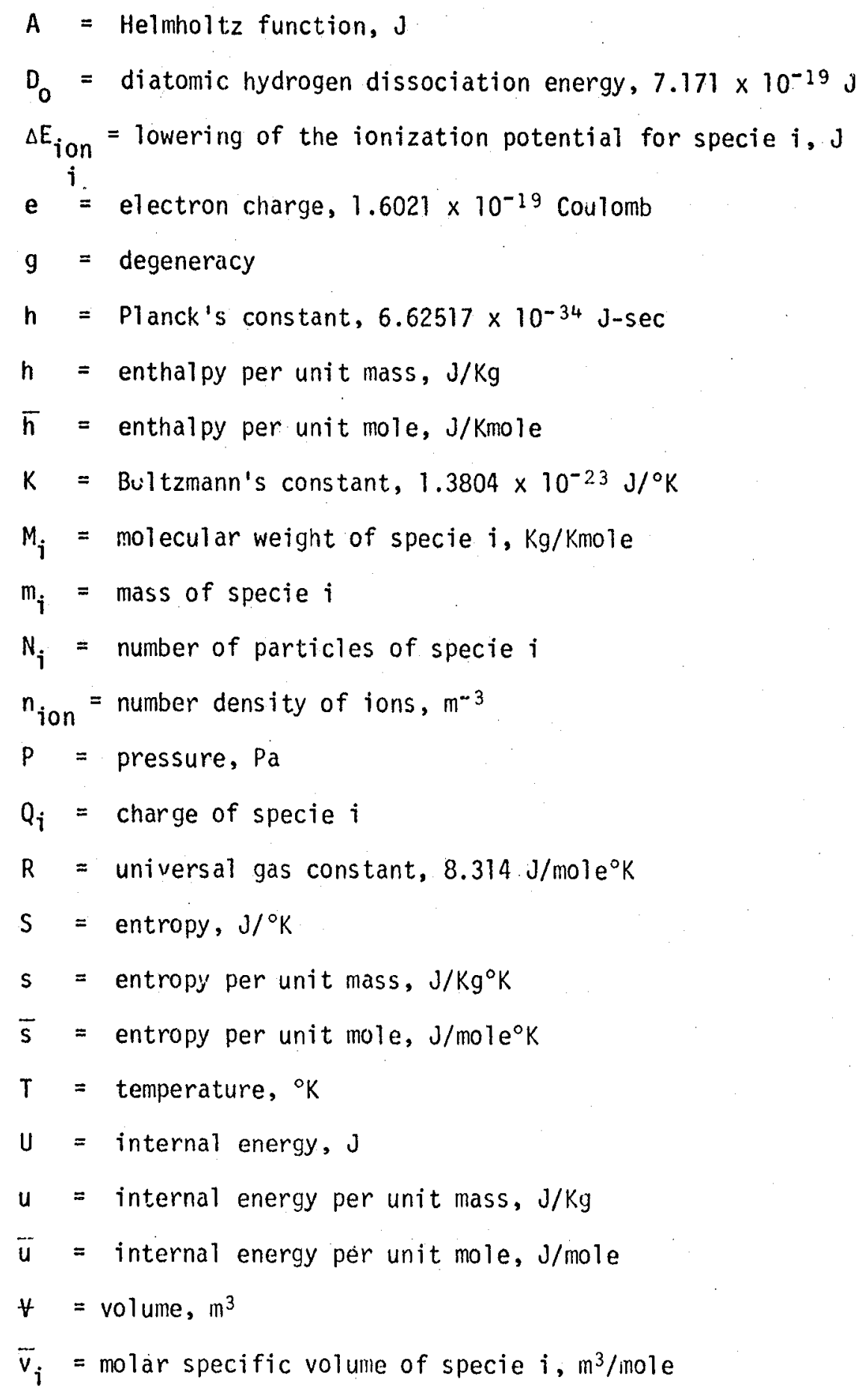




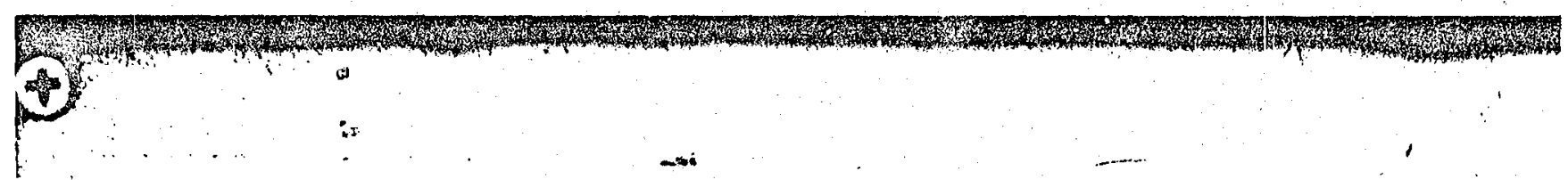

$Y_{i}=$ mole fraction of specie $i$

$z_{i}=$ partition function for specie $\mathbf{i}$

$\varepsilon_{j}=$ electronic energy of the $i^{\text {th }}$ level for a given specie, $J$

$\varepsilon_{i}=$ electron affinity for the $i^{\text {th }}$ specie, $J$

$\varepsilon_{i}=$ ionization energy for the $i^{\text {th }}$ specie, $J$ 


\section{APPENDIX B \\ EQUILIBRIUM HYOROGEN PROPERTIES}

- In analyzing ramjet operation it is necessary to calculate the equilibrium composition and thermodynamic properties for hydrogen. This is done by the methods presented in this Appendix. The species considered in this analysis are

$$
\mathrm{H}_{2}, \mathrm{H}_{2}^{+}, \mathrm{H}, \mathrm{H}^{+}, \mathrm{H}^{-}, \mathrm{e}^{-} \text {. }
$$

When a mixture of these species is at equilibrium the Helmholtz function

$$
A=U-T S
$$

is at a minimum. In this analysis it is assumed that the Helmholtz function can be expressed in the form

$$
A=A_{p g}+A_{C}
$$

where $A_{p g}$ is the perfect gas Helmholtz function, which is valid for noninteracting particles, and $A_{c}$ is the coulombic interaction Helmholtz function which comes into play for the case where the mixture is ionized. The coulombic term can be considered as a correction to the perfect gas form. The perfect gas term can be expressed in the form

$$
A_{p g}=-K T\left[\sum_{i} N_{i}\left(\ln \left(\frac{Z_{i}}{N_{i}}\right)+1\right)\right]
$$

where $N_{i}$ is the number of particles of the $i^{\text {th }}$ specie and $\left(Z_{i} / N_{i}\right)$ is the perfect gas partition function for the $i^{\text {th }}$ specie. The coulombic term 


\section{ORIGINAL PNCIE IY \\ OF POOR QUALITV}

can be expressed in the form

$$
A_{C}=-\frac{2}{3} e^{3}\left(\frac{\pi}{K T H}\right)^{\frac{1}{2}}\left(2 N_{j} Q_{i}^{2}\right)^{3 / 2}
$$

where $Q_{i}$ is the charge of the $i^{\text {th }}$ specie and $e$ is the charge on an electron. At equilibrium

$$
d A=d A_{p g}+d A_{c}=0
$$

The differentiation is taken to occur at a constant temperature and volume. Variations in the Helmholtz function due to changes in the composition are considered. The forms for the differentials are

$$
d A_{p g}=-K T \sum \ln \left(Z_{i} / N_{i}\right) d N_{i}
$$

and

$$
d A_{c}=-e^{3}\left(\frac{\pi}{K T H}\right)^{\frac{1}{2}}\left[\Sigma N_{j} Q_{i}^{2}\right]^{\frac{1}{2}}\left[\Sigma Q_{i}^{2} d N_{i}\right]
$$

Combining these expressions yields

$$
\sum \ln \frac{Z_{i}}{N_{i}} d N_{i}+\frac{e^{3}}{(K T)^{3 / 2}}\left(\frac{\pi}{\not}\right)^{\frac{1}{2}}\left[\Sigma N_{i} Q_{i}^{2}\right]^{\frac{1}{2}}\left[\Sigma Q_{i}^{2} d N_{i}\right]=0 .
$$

This expression can be put into a slightly different form

$$
\sum \ln \frac{Z_{i}}{N_{i}} d N_{i}+\sum\left\{\frac{e^{3}}{(K T)^{3 / 2}}\left(\frac{\pi}{\forall}\right)^{\frac{1}{2}}\left[\Sigma N_{i} Q_{i}^{2}\right]^{\frac{1}{2}} Q_{i}^{2} d N_{i}\right\}=0 .
$$

Factoring out $\mathrm{dN}_{\mathfrak{i}}$ yields

$$
\Sigma\left\{\ln \frac{Z_{i}}{N_{i}}+\frac{e^{3}}{(K T)^{3 / 2}}\left(\frac{\pi}{\forall}\right)^{\frac{1}{2}}\left[\Sigma N_{i} Q_{i}^{2}\right]^{1 / 2} Q_{i}^{2}\right\} d N_{i}=0
$$

or

$$
\sum J_{i} d N_{i}=0
$$


where

$$
J_{i} \equiv \ln \frac{Z_{i}}{N_{i}}+\frac{e^{3}}{(K T)^{3 / 2}}\left(\frac{\pi}{\forall}\right)^{3 / 2}\left[\Sigma N_{i} Q_{i}^{2}\right]^{\frac{1}{2}} Q_{i}^{2} .
$$

The chemical reactions considered are

$\begin{array}{lll}\rho_{\mathrm{a}} & \mathrm{H}_{2} \leftrightarrows 2 \mathrm{H} & \text { a) } \\ \rho_{\mathrm{b}} & \mathrm{H}_{2} \leftrightarrows \mathrm{H}_{2}^{+}+\mathrm{e}^{-} & \text {b) } \\ \rho_{\mathrm{c}} & H^{+} \leftrightarrows H^{+}+\mathrm{e}^{-} & \text {c) } \\ \rho_{\mathrm{d}} & H^{-} \leftrightarrows H+\mathrm{e}^{-} \cdot & \text { d) }\end{array}$

where the degree of reaction for each chemical reaction is defined as being the variable $\rho$. If each reaction is assumed to proceed slightiy from left to right then the change in each component is given by

$$
\begin{aligned}
& d N_{H_{2}}=-d \rho_{a}-d \rho_{b} \\
& d N_{H_{2}}+=d \rho_{b} \\
& d N_{H}=2 d \rho_{a}-d \rho_{c}+d \rho_{d} \\
& d N_{H^{+}}^{+}=d \rho_{c} \\
& d N_{H}^{-}=-d \rho_{d} \\
& d N_{e}^{-}=d \rho_{b}+d \rho_{c}+d \rho_{d} .
\end{aligned}
$$

By combining these expressior: with the expression

$$
\sum U_{i} d N_{i}=0
$$

the following equation can be obtained

$$
\begin{aligned}
& \left(-J_{H_{2}}+2 J_{H}\right) d \rho_{a}+\left(-J_{H_{2}}+J_{H_{2}}{ }^{+}+J_{e^{-}}\right) d \rho_{b}+ \\
& \left(-J_{H}+J_{H}+J_{e^{-}}\right) d \rho_{c}+\left(-J_{H}-J_{H}+J_{e^{-}}\right) d \rho_{d}=0 .
\end{aligned}
$$

Since the cnemical reactions and hence the $d \rho$ values are assumed to be 
inciependent, each quantity in brackets must be equal to zero at equilibrium. This equation is used to help determine the chemical equilibrium composition.

Consider the reiction

$$
\mathrm{H}_{2} \ddagger 2 \mathrm{H} \text {. }
$$

For this reaction the relation

$$
-\mathrm{J}_{H_{2}}+2 \mathrm{~J}_{H}=0
$$

is used. In more complete form this equation becomes

$$
\begin{aligned}
& \ln \frac{Z_{H_{2}}}{N_{H_{2}}}+\frac{e^{3}}{(K T)^{3 / 2}}\left(\frac{\pi}{\psi}\right)^{\frac{1}{2}}\left[\Sigma N_{i} Q_{i}^{2}\right]^{\frac{1}{2}} Q_{H_{2}}^{2} \\
& \quad=2 \operatorname{in} \frac{Z_{H}}{N_{H}}+\frac{2 e^{3}}{(K T)^{3 / 2}}\left(\frac{\pi}{\psi}\right)^{\frac{1}{2}}\left[\sum N_{i} Q_{i}\right]^{\frac{1}{2}} Q_{H}^{2}
\end{aligned}
$$

but

$$
Q_{H_{2}}=Q_{H}=\bar{c}
$$

so this equation degenerates to

$$
\frac{Z_{H}^{2}}{Z_{H_{2}}}=\frac{N_{H}^{2}}{N_{H_{2}}}
$$

which is the correct form for a conventional perfect gas anatys is. Multiplying each side of the equation by

$$
\frac{\Sigma N_{i}}{\left(\Sigma N_{i}\right)^{2}}=\frac{N}{N^{2}}
$$

allows this equation to be put inio the form 


\section{v}

$$
\frac{Y_{H}^{2}}{Y_{H_{2}}}=\frac{(Z / N)_{H}^{2}}{(Z / N)_{H_{2}}}
$$

where $N$ is total number of particles.

For the reaction

$$
\mathrm{H}_{2} \stackrel{\leftarrow}{\sharp} \mathrm{H}_{2}^{+}+\mathrm{e}^{-}
$$

the relation

$$
-\mathrm{J}_{\mathrm{H}_{2}}+\mathrm{J}_{\mathrm{H}_{2}}+\mathrm{J}_{\mathrm{e}^{-}}=0
$$

is valid. In its more complete form this equation becomes

$$
\begin{gathered}
\text { in } \frac{Z_{H_{2}}}{N_{H_{2}}}+\frac{e^{3}}{(K T)^{3 / 2}}\left(\frac{\pi}{\forall}\right)^{\frac{1}{2}}\left[\Sigma N_{i} Q_{i}^{2}\right]^{\frac{1}{2}} Q_{H_{2}}^{2}= \\
\text { sn } \frac{Z_{H_{2}}{ }^{+}}{N_{H_{2}}{ }^{+}}+\ln \frac{Z_{e^{-}}}{N_{e^{-}}}+\frac{e^{3}}{(K T)^{3 / 2}}\left(\frac{\pi}{\forall}\right)^{\frac{1}{2}}\left[Q_{H_{2}}^{2}+Q_{e}^{2}-\right] \cdot\left[\sum N_{i} Q_{i}^{2}\right]^{\frac{1}{2}}
\end{gathered}
$$

where

$$
\begin{gathered}
Q_{H_{2}}=0 \\
Q_{H_{2}}+=+1 \\
Q_{e}=-1 .
\end{gathered}
$$

After rearrangement this equation degenerates to

$$
\ln \left\{\left(\frac{Z_{H_{2}}}{Z_{H_{2}} Z_{e}=}\right)\left(\frac{{ }^{i} H_{2}^{+}}{N_{e}}\right)\right\}=2 e^{3} \frac{\pi^{3 / 2}}{(K T)^{3 / 2}} n_{\text {ion }}^{\frac{1}{2}}
$$

where the parameter $n_{i 0 n}$ is defined by

$$
n_{\text {ion }}=\frac{\sum N_{i} Q_{i}^{2}}{\forall}
$$


and represents the ion number density. Rearranging and multiplying through by $N / N^{2}$ yields

$$
\frac{Y_{H_{2}+Y^{-}}}{Y_{H_{2}}}=\frac{(Z / N)_{H_{2}^{+}}(Z / N)_{e^{-}}}{(Z / N)_{H_{2}}} \exp \left\{\left(\frac{2 e^{3} \pi^{\frac{1}{2} n_{i o n}^{\frac{1}{2}}}}{(K T)^{\frac{1}{2}}}\right)\left(\frac{1}{K T}\right)\right\} .
$$

For the case where $n_{i o n}$ is a small number, the exponential term on the right hand side of the above equation will be close to one, which corresponds to the form obtained when only a perfect gas analysis is done (i.e. the Saha Equation). When the number density of ions is significant, however, a coulombic field will be present, and this field will affect the ionization of the different particles. The net effect of this coulombic field is to decrease the amount of energy needed to remove an electron from the influence of a given nucleus (the ionization energy is decreased). Consequently, the exponential term in the preceding equation can be thought of as representing an expression for the lowering of the ionization potential. The lowering of the ionization potential can therefore be expressed as

$$
\underset{\substack{\Delta \mathrm{H}_{2} \\ \mathrm{H}_{2}}}{ }=2 \mathrm{e}^{3}\left(\frac{\pi \mathrm{n}_{\text {ion }}}{\mathrm{KT}}\right)^{\frac{1}{2}}
$$

and the expression for the mole fraction ratio becomes

$$
\frac{Y_{H_{2}}+Y_{e^{-}}}{Y_{H_{2}}}=\frac{(Z / N)_{H_{2}}(Z / N) e^{-}}{(Z / N)_{H_{2}}} \exp \left(\frac{\begin{array}{c}
\Delta E_{i \cap n} \\
H_{2}
\end{array}}{K T}\right) .
$$

For the chemical reaction

$$
H^{*} \rightarrow H^{+}+e^{-}
$$


the same form is obtained for the expression for the mole fraction ratio namely

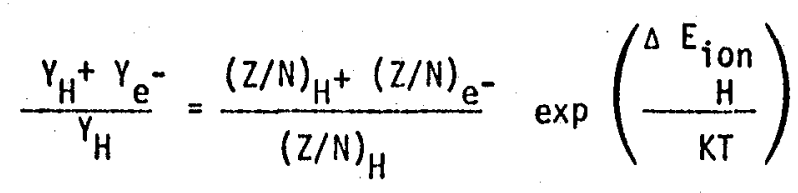

where

$$
\Delta \underset{H}{E_{\text {ion }}}=2 e^{3}\left(\frac{\pi n_{\text {ion }}}{K T}\right)^{\frac{i}{2}}=\Delta \underset{\substack{H_{2} \\ H_{2}}}{ } .
$$

An interesting result is obtained when the reaction

$$
\mathrm{H}^{-} \leftrightarrows \mathrm{H}+\mathrm{e}^{-}
$$

is considered. For this reaction the relation

$$
\mathrm{J}_{H^{-}}=\mathrm{J}_{H}+\mathrm{J}_{\mathrm{e}^{-}}
$$

is valid. When this equation is expanded it assumes the following form

$$
\begin{aligned}
& \ln \frac{Z_{H^{-}}}{N_{H^{-}}}+\frac{e^{3}}{(K T)^{3 / 2}}\left(\frac{\pi}{\psi}\right)^{\frac{1}{2}}\left[\Sigma N_{i} Q_{i}^{2}\right]^{\frac{1}{2}} Q_{H_{2}^{-}}^{2}= \\
& \ln \frac{Z_{H}}{N_{H}}+\ln \frac{Z_{e^{-}}}{N_{e^{-}}}+\frac{e^{3}}{(K T)^{3 / 2}}\left(\frac{\pi}{\psi}\right)^{\frac{1}{2}}\left[\Sigma N_{i} Q_{i}^{2}\right]^{\frac{1}{2}} Q_{e^{-}}^{2}
\end{aligned}
$$

where

$$
Q_{\mathrm{e}^{-}}^{2}=Q_{\mathrm{H}^{-}}^{2}=1 .
$$

The coulombic interaction terms will cancel so this equation assumes the form

$$
\frac{Z_{H} Z_{e^{-}}}{Z_{H^{-}}}=\frac{N_{H^{-}} N_{e^{-}}}{N_{H^{-}}}
$$




\section{2}

which predicts that, for a negative ion, there is no lowering of the ionization potential. However, if the negative ion was doubly charged and only one electron was removed, then a change in the ionization potential would be predicted. Multiplying the previous equation by $N / N^{2}$ yields

$$
\frac{Y_{H^{Y}} \mathrm{e}^{-}}{Y_{H^{-}}}=\frac{(Z / N)_{H}(Z / N)_{\mathrm{e}^{-}}}{(Z / N)_{H^{-}}} .
$$

At this point in the analysis, four equations describing the relationships of the mole fraction species have been derived. In summary, these equations are

$$
\begin{aligned}
& \frac{\left(Y_{H}\right)^{2}}{Y_{H_{2}}}=\frac{(Z / N)_{H}^{2}}{(Z / N)_{H_{2}}} \\
& \frac{Y_{H_{2}^{+}} Y_{\mathrm{e}^{-}}}{Y_{\mathrm{H}_{2}}}=\frac{(Z / N)_{H_{2}^{+}}(Z / N) \mathrm{e}^{-}}{(Z / N)_{H_{2}}} \exp \left[\frac{\begin{array}{r}
\Delta E_{\text {ion }} \\
H_{2}
\end{array}}{K T}\right] \\
& \frac{Y_{H^{+}} Y_{e^{-}}}{Y_{H}}=\frac{(Z / N)_{H^{+}}(Z / N)_{e^{-}}}{(Z / N)_{H}} \exp \left[\frac{\begin{array}{c}
\Delta E_{i o n} \\
H
\end{array}}{K T}\right] \\
& \frac{Y_{H} Y_{e^{-}}}{Y_{H^{-}}}=\frac{(Z / N)_{H}(Z / N)_{e^{-}}}{(Z / N)_{H^{-}}} .
\end{aligned}
$$

However, because there are six species considered, two more equations must be obtained in order to ensure a unique solution. One of these two equations can be derived by assuming that charge is conserved

$$
Y_{H^{+}}+Y_{H_{2}}+Y_{\mathrm{e}^{-}}-Y_{H^{-}}=0 .
$$


The second equation comes from summing up the mole fractions

$$
Y_{H_{2}}+Y_{H_{2}^{+}}+Y_{H^{+}}+Y_{H^{+}}+Y_{H^{-}}+Y_{e^{-}}=\frac{\Sigma n_{j}}{n}=1 \text {. }
$$

The variable $(Z / N)_{i}$ is the partition function for the $i^{\text {th }}$ specie evaluated at the total system pressure and temperature. In order to evaluate the partition function it is necessary to select an energy datum, and the datum selected for this analysis is the ground electronic state of the $\mathrm{H}_{2}$ molecule at $0^{\circ} \mathrm{K}$. The partition function of each specie is computed relative to this state.

The partition function for any specie $i$ can be expressed in the form

$$
(Z / N)_{i}=\left(\frac{z_{t}}{N}\right){ }_{i}\left(z_{\text {internal }}\right)_{i}
$$

where the cranslational contribution is given by

$$
\left(z_{t} / N\right)_{i}=\left(\frac{2 \pi m_{i}}{h^{2}}\right)^{3 / 2} \frac{(K T)^{5 / 2}}{p} .
$$

The form of the internal contribution varies depending on whether the specie under consideration is diatomic or monatomic. A diatomic specie will have rotational and vibrational contributions, and the diatomic species and some of the monatomic species will have electronic contributions. The possibility of having a chemical energy contribution to the internal partition function also exists for some of the monatomic and diatomic species, notably $\mathrm{H}_{2}^{+}, \mathrm{H}, \mathrm{H}^{+}, \mathrm{H}^{-}$, and $\mathrm{e}^{-}$, depending on where the ground state energy of the given specie relative to the datum selected above lies. 
Consider the monatomic specie of neutral hydrogen $(H)$. The internal partition function for $H$ is given by

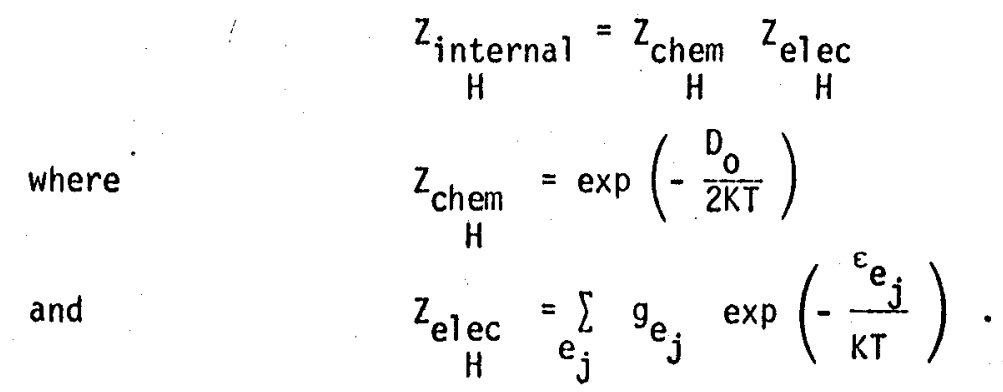

This expression assumes that each $H$ atom receives $1 / 2$ of the chemical energy required to dissociate a ground state $\mathrm{H}_{2}$ molecule. The degeneracy $\left(\mathrm{g}_{\mathrm{e}_{j}}\right)$ in the electronic contribution relation is given by

$$
g_{e_{j}}=2 j^{2} \quad j=1,2,3, \ldots
$$

and the energy $\left(\varepsilon_{e_{j}}\right)$ is given by

$$
\varepsilon_{j}=\left(\frac{2 \pi^{2} C^{2} L^{2} e^{4} m}{h^{2}}\right) \frac{j^{2}-1}{j^{2}} \quad j=1,2,3, \ldots
$$

In this equation for the energy state, $L$ is the charge of the nucleus ( 1 for $H$ ), e is the electron charge, $m$ is the electron mass, $h$ is Planck's constant and $C$ is a proportionality constant. Inserting these relations into the expression for the electronic partition function for the hydrogen $(H)$ atom yieids

$$
Z_{\text {elec }}=\sum_{j=1}^{J} 2 j^{2} \exp \left[\frac{2 \pi^{2} C^{2} L^{2} e^{4} m}{h^{2} K T} \frac{1-j^{2}}{j^{2}}\right]
$$




\section{5}

Examination of this equation brings to light the fact that the series will diverge uniess a cutoff $(J)$ for the summation is specified. A method of calculating this cutoff will be given later in this Appendix.

The other monatomic species are $\mathrm{H}^{+}, \mathrm{e}^{-}$and $\mathrm{H}^{-}$. For the $\mathrm{H}^{+}$particle the value for the internal partition function is 1 , and the chemical partition function is given by

$$
\underset{H^{+}}{Z_{\text {chem }}}=\exp \left[\frac{-\left(D_{0} / 2+\varepsilon_{i_{H}}\right)}{K T}\right] \text {. }
$$

In this expression $\varepsilon_{i_{H}}$ is the ionization energy of a hydrogen atom. The $\mathrm{H}^{+}$particle is assumed to carry all of the ionization energy. For the electron $\left(\mathrm{e}^{-}\right)$the internal partition function is

$$
z_{\text {int }}=2
$$

because of the spin contribution to the degeneracy. The electron is assumed to carry no chemical energy so the chemical contribution to the partition function is

$$
z_{\text {chem }^{-}}=1
$$

The $H^{-}$ion has an electron affinity value ( $\varepsilon_{\text {affin }}$ ) that must be taken into account when calculating the chemical partition function. The chemical partition function is given by

$$
Z_{\text {chem }}=\exp \left[\frac{{ }_{H^{-}}\left(0_{0} / 2+\varepsilon_{\text {affin }}\right)}{K T}\right] \text {. }
$$

Only the ground electronic state is considered for the $\mathrm{H}^{-}$ion and 
the internal partition function (electronic) is

$$
Z_{\text {int }}=1 .
$$

Higher electronic states are neglected because the existence of electronically excited negative ions is rare. ${ }^{B 1}$ The binding energy for a negative ion is generally small and it is not too difficult to remove an electron from a negatively charged particle. Also, because the binding energy is small, it seems to make sense to neglect electronic states above the ground state because the higher el ectronic states effectively reduce the amount of energy required to remove an electron. The binding energy for the extra electron on a negative ion is not large because the inner electron(s) repel the extra electron with a charge that is equal to the charge of the nucleus, effectively shielding the extra electron from the nucleus.

For the diatomic species $\left(\mathrm{H}_{2}, \mathrm{H}_{2}{ }^{+}\right)$there are rotational and vibrational contributions to the internal partition functions that must be taken into account. In this analysis coupling between the rotational and vibrational enargy storage modes is allowed, and an expression characterizing the rutational and vibrational modes for separate electronic levels is presented later in this Appendix. The complete internal partition function for the $\mathrm{H}_{2}$ molecule (and $\mathrm{H}_{2}{ }^{+}$) is expressed by

$$
z_{i n t}=\sum_{e_{j}} z_{r v} e_{j} e_{j}
$$

where the summation must be terminated at a finite electronic energy level. The internal partition function is more completely expressed in 


\section{ORIGINAL PACE IS \\ 77}

the form

$$
\begin{gathered}
z_{i n t}=\underset{e_{j}}{e_{j}} g_{e_{j}} \exp \left(\frac{-\varepsilon}{k T}\right) \frac{1}{\sigma y}\left(\frac{1}{1-e^{-t}}\right) \\
\left\{1+\frac{y}{3}+\frac{2 \gamma}{y}+\frac{\delta}{e^{t}-1}+\frac{2 x^{*} t}{\left(e^{t}-1\right)^{2}} \mid .\right.
\end{gathered}
$$

In this expression $\sigma=2$ because a diatomic molecule is symetric. The following parameters are evaluated from the rotational and vibrational constants for the given electronic levels (the rotational and vibrational constants can be found in compilations such as the one in Reference B2).

$$
\begin{aligned}
& t=\frac{h_{C}}{K T}\left(W_{\varepsilon}-x_{\varepsilon} W_{\varepsilon}\right) \\
& x^{*}=\frac{X_{\varepsilon} W_{\varepsilon}}{W_{\varepsilon}-X_{\varepsilon} W_{\varepsilon}} \\
& y=\frac{h_{C}}{K T}\left(B_{\varepsilon}-1 / 2 u_{\varepsilon}\right) \\
& \delta=\frac{\alpha}{B_{\varepsilon}-1 / 2 \alpha_{\varepsilon}} \\
& \gamma=\frac{D_{\varepsilon}}{B_{\varepsilon}-1 / 2 \alpha_{\varepsilon}} .
\end{aligned}
$$

The parameters $W_{\varepsilon}, X_{E} W_{E}, B_{\varepsilon}$, and $\alpha_{\varepsilon}$ are the rotational and vibrational coilstants and $D_{\varepsilon}$ is computed using

$$
D_{\varepsilon}=\frac{4 B_{\varepsilon}^{3}}{W_{\varepsilon}^{2}} .
$$


The $\mathrm{H}_{2}{ }^{+}$molecule has a chemical (ionization) energy that must be taken into account, and the chemical partition function for $\mathrm{H}_{2}^{+}$is given by

$$
Z_{\text {chem }}=\exp \left(\frac{{ }_{H_{2}{ }^{+}}^{-\varepsilon_{i_{H}}}}{K T}\right) \text {. }
$$

It should be explained here that the ionization energies used in evaluating the partition functions are values published in a reference such as Reference B3. It is assumed that these values were obtained for rarified, very weakly ionized gases where coulombic interactions are unimportant. The calculation of the ionization energy for a given specie takes the published value as a baseline, and this value is then changed in the manner detailed earlier where the changes in ionization energies are defined for the different species.

In the evaluation of the various partition functions it is necessary to specify cutoff values whenever electronic levels other than the ground level are considered. In essence, the problem is establishing a criterion for when an electron can no longer be considered to be bound to the nucleus of an atom or to the nuclei of a molecule. The criterion selected for this analysis is to not allow any electronic energies which exceed the ionization energy (taking into account the lowering of the ionization energy discussed earlier).

Solving for the equilibrium composition is an iterative process because the eouations are noilinear and the lowering of the ionization potential must be determinec implicitly. The method used in this analys is is to assume initially that no ions are present and to apply the model just presented and compute the ion density. A new ion density based on the computed value is then used as input and this process 
continues until the difference between the assumed and computed ion density is sufficiently small. The value required for the number density of the ions $\left(n_{i o n}\right)$ is computed from

$$
n_{\text {ion }}=\frac{N_{\text {ion }}}{\forall}=\frac{N}{\forall} \frac{N_{\text {ion }}}{N}=\frac{P}{K T}\left(Y_{e^{-}}+Y_{H^{-}}+Y_{H^{+}}+Y_{H_{2}}\right) .
$$

Once the equilibrium composition has been found, the thermodynamic properties of the mixture must aiso be evaluated. It is noted that ine coulombic corrections previousiy referred to in this Appendix will affect the composition and the thermodynamic properties of the species because the ionization energies and the allowed electronic excitation levels are changed by allowing coulombic corrections to appear. Also, the coulomb field itself will possess definite thermodynamic properties and these properties will contribute to the themodynamic state of the complete hydrogen mixture. For example, the ions will be slightly attracted to each other so that the actual system pressure will be slightly less than is predicted by the ideal gas equation. However, in the spirit of Reference $B A$, it is assumed in this analysis that the contribution of the colilombic field to the total system themodynamic state of a hydrogen mixture is small and can therefore be neglected without introducing significant error.

The entropy $(\bar{s})$, enthalpy $(\bar{h})$ and internal energy $(\bar{u})$ of a mixture can be expressed on a molar basis by the following relations

$$
\begin{aligned}
& \bar{s}=\sum Y_{i} \vec{s}_{i} \\
& \bar{h}=\sum Y_{i} \bar{n}_{i} \\
& \bar{u}=\sum Y_{i} \bar{u}_{i} .
\end{aligned}
$$


Dividing each equation through by the average molecular weight which is defined by

$$
M_{\text {ave }}=\sum Y_{\mathbf{i}} M_{\mathbf{i}}
$$

allows these properties to be expressed on a per unit mass basis

$$
\begin{aligned}
& s=\frac{\sum Y_{i} \ddot{s}_{i}}{\sum Y_{i} M_{i}} \\
& n=\frac{\sum Y_{i} \bar{r}_{i}}{\sum Y_{i} \bar{M}_{i}} \\
& u=\frac{\sum Y_{i} \bar{u}_{i}}{\sum Y_{i} \bar{M}_{i}} .
\end{aligned}
$$

The internál energy on a molar basis of a specie $i\left(\bar{u}_{i}\right)$ can be expressed in the form

$$
\bar{u}_{i}=R T^{2}\left(\frac{\partial \ln z_{i}}{\partial T}\right)_{\forall_{i}} .
$$

Since the enthalpy $\left(\bar{h}_{i}\right)$ is defined by

$$
\bar{h}_{i}=\bar{u}_{i}+P_{i} \bar{v}_{i}
$$

where $\bar{v}_{i}$ is the molar specific volume, and the ideal gas equation

$$
P_{i} \bar{v}_{i}=R T
$$

is assumed to be valid, the enthalpy per mole can be expressed in the form

$$
\bar{n}_{i}=R T^{2}\left(\frac{\partial \ln Z_{i}}{\partial T}\right)_{\forall_{i}}+R T .
$$




\section{1}

ORIGINAL PAGE IS
The entropy per unit mole is expressed by OF POOR QUALITY

$$
\bar{s}_{i}=R\left|\ln \frac{z_{i}}{N_{i}}+1\right|+\frac{\bar{u}_{i}}{T} .
$$

The variable $R$ is the universal gas constant.

In order that the thermodynamic properties can be calculated the partial derivative appearing in the thermodynamic relations is expressed in the form

$$
\left(\frac{\partial\left(\operatorname{sn} z_{i}\right)}{\partial T}\right)_{\forall_{i}}=\frac{1}{Z_{i}}\left(\frac{\partial z_{i}}{\partial T}\right)_{\forall_{i}}
$$

It is possible to separate the translational partition function out of this expression. The partial derivative of the translational partition function for any specie is given by

$$
\frac{1}{Z_{\text {trans }}}\left(\frac{\partial Z_{\text {trans }}}{\partial T}\right)_{\forall_{i}}=\frac{3}{2} \frac{1}{T} .
$$

Consequeitly, the translational contributions to the internal energy and enthalpy are given by

$$
\begin{aligned}
& \bar{u}_{i}=3 / 2 \mathrm{RT} \\
& \text { trans } \\
& \bar{h}_{i}=5 / 2 \mathrm{RT} . \\
& \text { trans }
\end{aligned}
$$

When the chemical energy is considered, the internal energy and enthalpy are given by

$$
\bar{u}_{i}=\bar{h}_{i}=N_{0}\left(D_{0} / 2+\varepsilon_{i}\right)
$$

where $D_{0}$ is the dissociation energy and $\varepsilon_{i}$ is the ionization energy. 


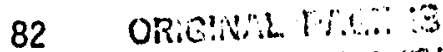 \\ of POOR Quning}

The electronic contribution to the themodynamic preoperties must be accounted for in the monatomic hydrogen $(H)$ atom. The form for the partial 'erivative is

$$
\frac{\partial\left(\ln Z_{e l e c}\right)}{\partial T}=\frac{E}{K T^{2}} \frac{\sum_{j=1}^{J}\left(j^{2}-1\right) \exp \left(\frac{-F}{K T} \frac{j^{2}-1}{j^{2}}\right)}{\sum_{j=1}^{J} j^{2} \exp \left(\frac{-F}{K T} \frac{j^{2}-1}{j^{2}}\right)} .
$$

The value obtained in this summation is utilized to calculate the enthalpy $(\bar{h})$ and the internal energy $(\bar{u})$ for the hydrogen atom. The constant $F$ is given by

$$
F=\frac{2 \pi^{2} C^{2} L^{2} e^{4} m}{h^{2}}
$$

For the diatomic species the internal contributions to the partition function are given by

$$
z_{i n t}=e_{j} z_{r v} e_{j} e_{j}
$$

Consequently the partial derivative is evaluated in the form

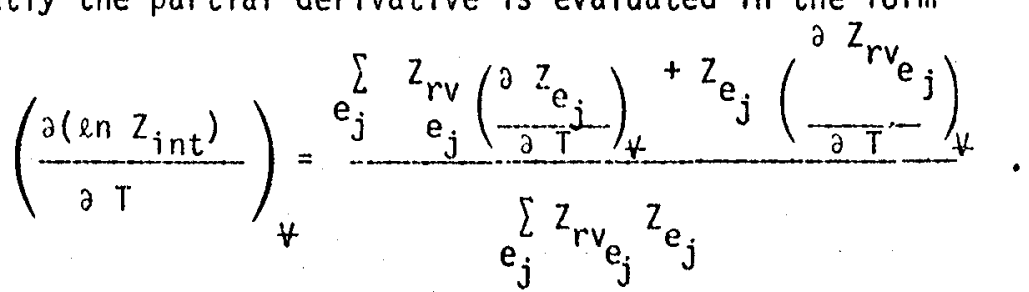

For a given electronic energy $e_{j}$ the partial derivative of the electronic contribution function is given by 


\section{ORIGINAL PACE IJ OF POOR QUAAITY}

$$
\left(\frac{\partial Z_{e_{j}}}{\partial T}\right)_{\forall}=g_{j} \frac{\varepsilon_{j}}{K T^{2}} \exp \left(-\frac{\varepsilon_{j}}{K T}\right) .
$$

The expression for the partial derivative of the rotational-vibrational term is given below

$$
\frac{\partial\left(z_{r v}\right)}{\partial T} e_{j}=A B+C D
$$

where the paranleters $A, B, C$, and $D$ are evaluated using

$$
\begin{aligned}
& A=\frac{1}{\sigma y} \frac{1}{1-e^{-t}} \\
& B=\frac{1}{T}\left\{\frac{y}{3}+\frac{2 y}{y}+\frac{t\left(\delta e^{t}-2 x^{*}\right)}{\left(e^{t}-1\right)^{2}}+\frac{4 x^{*} t^{2} e^{t}}{\left(e^{t}-1\right)^{3}}\right\} \\
& C=1+\frac{y}{3}+\frac{2 y}{y}+\frac{\delta}{e^{t}-1}+\frac{2 x^{\star} t}{\left(e^{t}-1\right)^{2}} \\
& D=\frac{1}{\sigma y T} \frac{1+e^{-t}(t-1)}{\left(1-e^{-t}\right)^{2}} .
\end{aligned}
$$

Figures $B 1, B C$ and $B 3$ illustrate results obtained for the mole fractions of the major constituents of equilibrium hydrogen for different pressures and temperatures. The constituents $\mathrm{H}_{2}{ }^{+}$and $\mathrm{H}^{-}$were never signiticant so results for these species are not shown. It is noted that even for the 10 atmosphere case (Fiq. Bl) ionization does not begin to become significant until the temperature approaches 12,000 Kelvin. As a test, the calculated values for the thermodynamic properties and the composition were 


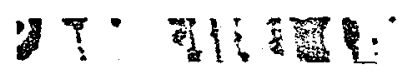

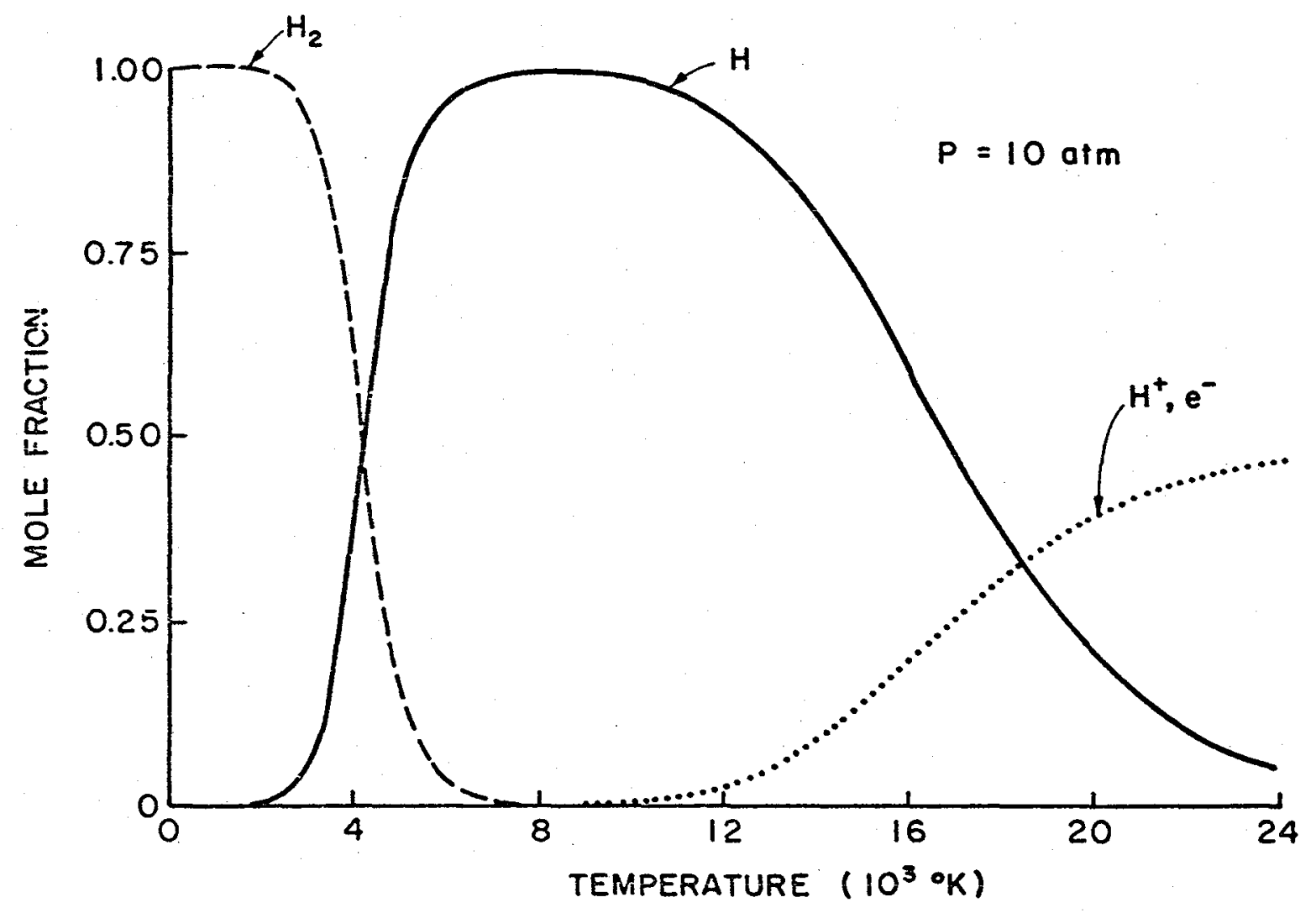

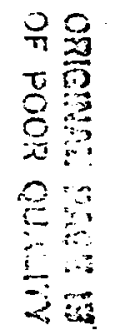

Fig. B1 Equilibrium Hydrogen Composition at $10 \mathrm{Atm}$ 

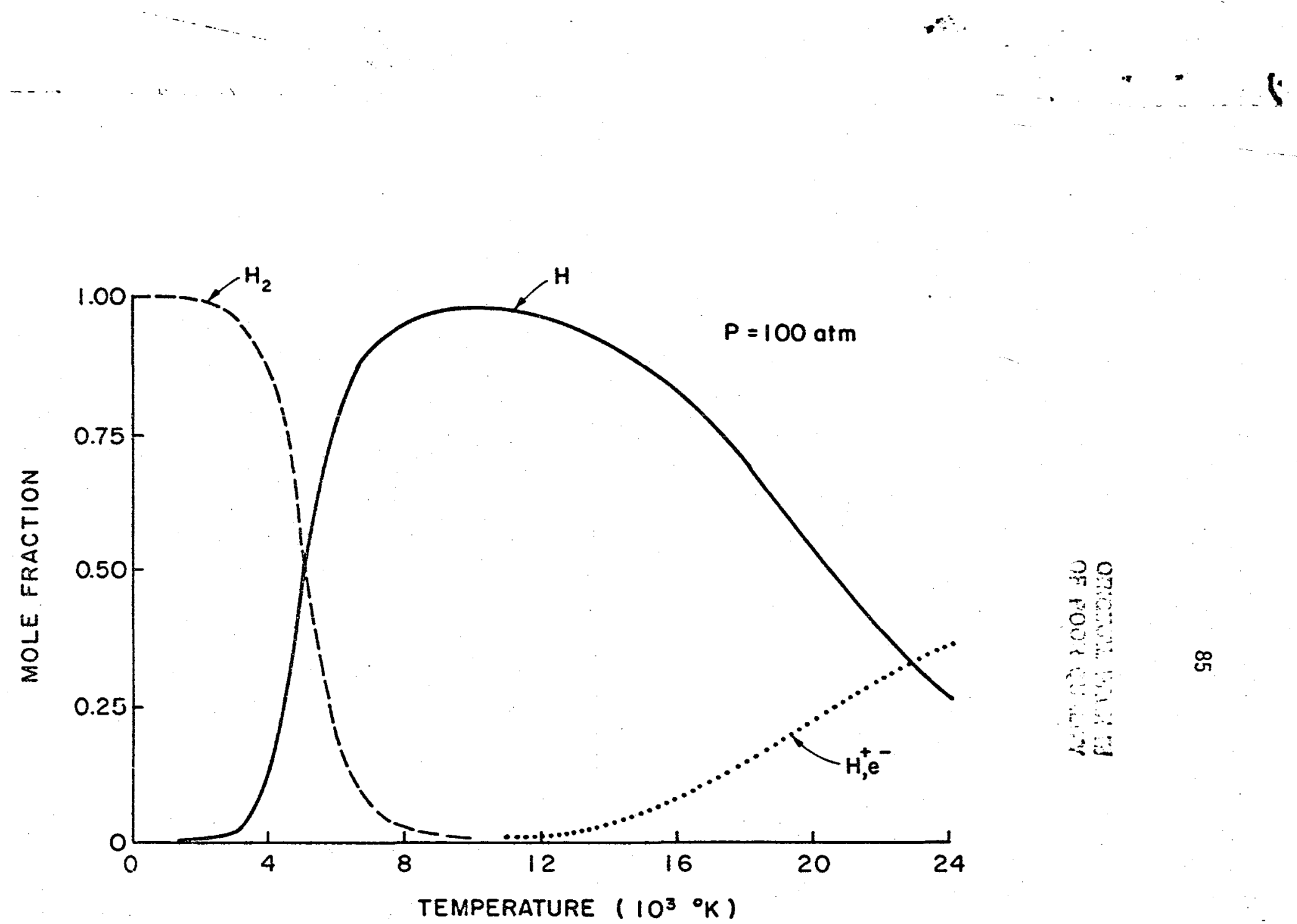

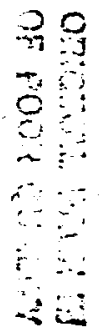

Fig. B2 Equilibrium Hydrogen Composition at $100 \mathrm{Atm}$ 
b) -

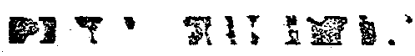

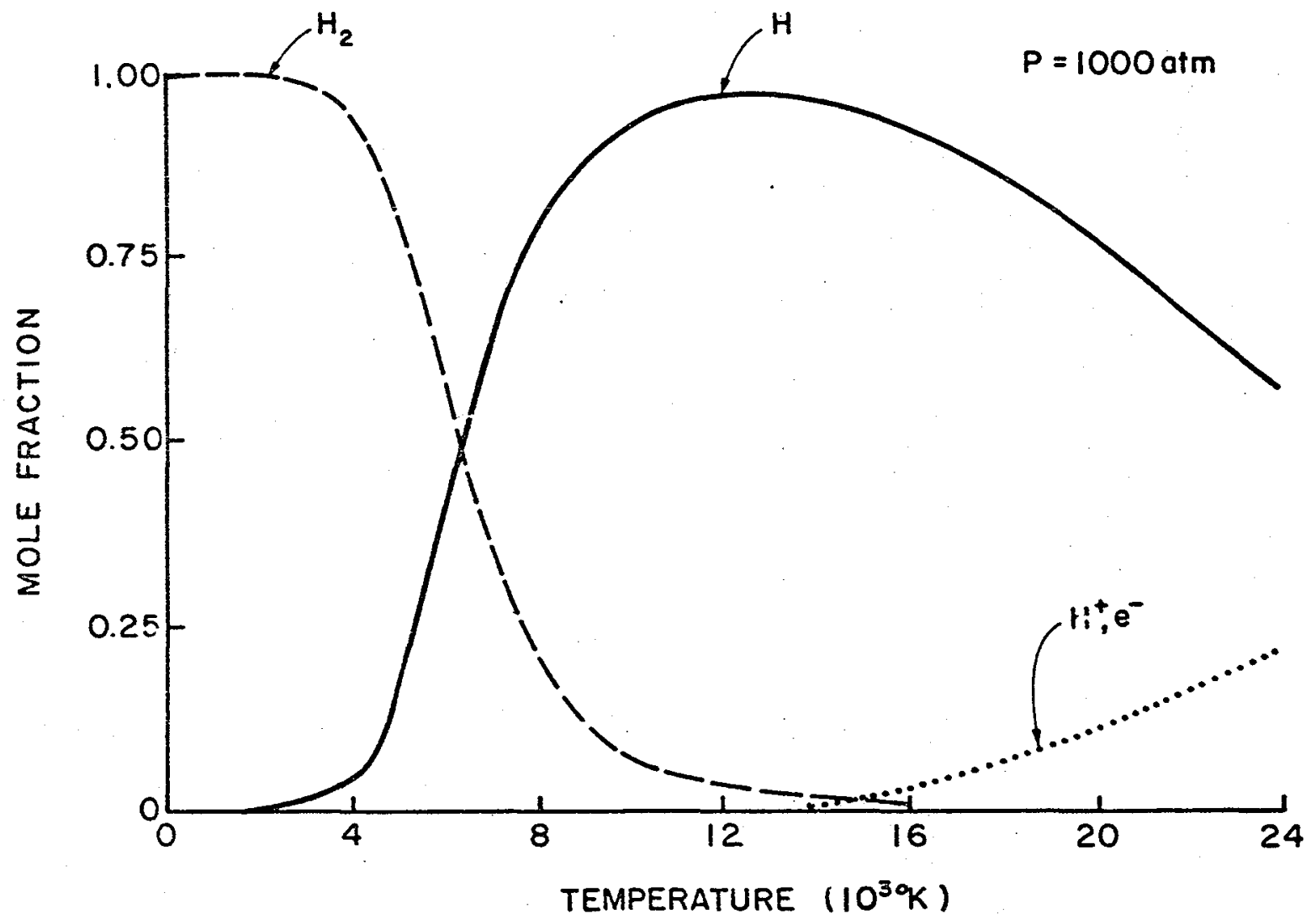

Fig. B3 Equilibrium Hydrogen Composition at $1000 \mathrm{Atm}$ 


\section{1}

$\checkmark$

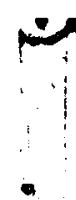

compared to values obtained from Reference B5. In the cases evaluated, the percent differences between the results from Reference $B 5$ and the results calculated were typicall of the order of $1 \%$ to $3 \%$. It is recognized that the results of Reference 85 doe not include ionization. However, the excellent agreement between the low-temperature results presented in Reference 85 and those given by the model described here is assumed to indicate high accuracy in the high-temperature regimes as well.

The electrothermal ramjet was typically found to be operating within internal propellant temperature regimes of the order of $10^{3}$ Kelvin and pressure regimes of the order of $10^{3}$ atmospheres and it was found that ionization was typically unimportant for the ramjet operational conditions presented in this thesis. 
APPENDIX C

NONEQUILIBRIUM NOZZLE FLOW

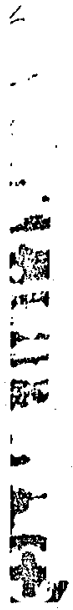


APPENDIX C NOMENCLATURE

$B=$ constant used in Eq. 8,78.01 $\frac{\mathrm{Kmole}}{\mathrm{m}^{3} \mathrm{~K}^{\frac{1}{2}}}$

$C_{H}=$ mass fraction of $H$

$D_{0}=H_{2}$ dissociation energy, $7.171 \times 10^{-19} \mathrm{~J}$

$H=$ monatomic hydrogen specie

$\mathrm{H}_{2}=$ diatomic hydrogen specie

$K=$ Boltzmann's constant, $1.3804 \times 10^{-23} \mathrm{~J} /{ }^{\circ} \mathrm{K}$

$K_{b}=$ backward rate constant, $\frac{m^{6}}{k m o l e^{2} \mathrm{sec}}$

$K_{f}=$ forward rate constant, $\frac{m^{3}}{\text { Kmole sec }}$

$M_{H}=$ molecular weight of $H, \frac{\mathrm{Kg}}{\mathrm{Kmole}}$

$N=$ constant used in Equation 9

$R_{d}=$ dissociation rate, $\frac{K m o l \epsilon}{m^{3} \mathrm{sec}}$

$R_{r}=$ recombination rate, $\frac{\mathrm{Kmole}}{\mathrm{m}^{3} \mathrm{sec}}$

$T=$ temperature, ${ }^{\circ} \mathrm{K}$

$t=$ time, sec

$u=$ velocity, $\mathrm{m} / \mathrm{s}$

$\forall \quad=$ volume, $\mathrm{m}^{3}$

$X=$ distance, $m$

$\rho=$ density, $\mathrm{Kg} / \mathrm{m}^{3}$

$\theta_{v}=$ harmonic oscillator constant for $\mathrm{H}_{2}, 6338.3^{\circ} \mathrm{K}$ 


\section{APPENDIX C \\ NONEQUILIBRIUM NOZZLE FLOW}

In the analysis of supersonic nozzles it is desirable to evaluate the nonequilbrium effects caused by finite rate reactions. This Appendix describes the Bray sudden freezing technique ${ }^{\mathrm{Cl}}$ which was utilized in the analyses presented in this thesis.

The dominant species in this analysis are assumed to be $\mathrm{H}_{2}$ and $\mathrm{H}$ and the following chemical reactions are considered

$$
\begin{aligned}
& \mathrm{H}_{2}+\mathrm{H}_{2} \stackrel{\leftarrow}{\rightarrow} \mathrm{H}+\mathrm{H}_{2} \\
& \mathrm{H}_{2}+\mathrm{H} \leftrightarrows 2 \mathrm{H}+\mathrm{H} .
\end{aligned}
$$

Four body and higher collisions are neglected. The rate equations which are assumed to apply to the above chemical reactions are for Eq. 1

$$
\frac{D[H]}{D t}=\underset{\mathrm{H}_{2}}{2 \mathrm{~K}_{f}}\left[\mathrm{H}_{2}\right]^{2}-\underset{\mathrm{b}}{2 \mathrm{H}}[\mathrm{H}]^{2}\left[\mathrm{H}_{2}\right]
$$

and for Eq. 2

$$
\frac{D[H]}{D t}=\underset{H}{2 K_{f}}[H]\left[H_{2}\right]-\underset{H}{2 K_{b}}[H]^{3} .
$$

[H] and $\left[\mathrm{H}_{2}\right]$ represent the molar densities of $\mathrm{H}$ and $\mathrm{H}_{2}$ and $\mathrm{K}_{f}$ and $\mathrm{K}_{b}$ are the correspunding forward and backward rate constants pertaining to Eq. 1. Similarly $K_{f_{H}}$ and $K_{b_{H}}$ correspond to Eq. 2. Combining these equations to obtain the net molar production rate for atomic hydrogen yields

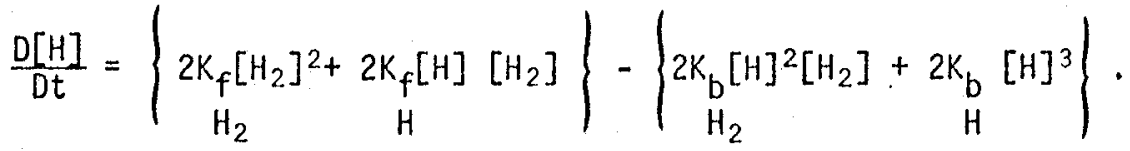


Defining

$$
\begin{aligned}
& R_{d}=\underset{H_{2}}{2 K_{f}}\left[H_{2}\right]^{2}+2 K_{f_{H}}[H]\left[H_{2}\right] \\
& R_{r}=\underset{H_{2}}{2 K_{t}}[H]^{2}\left[H_{2}\right]+2 K_{b_{H}}[H]^{3}
\end{aligned}
$$

where $R_{d}$ represents the rate of dissociation and $R_{r}$ represents the rate of recombination permits Eq. 5 to be expressed in the form

$$
\frac{D[H]}{D t}=R_{d}-R_{r} \text {. }
$$

It should be made clear at this point in the analys is that the previous equation assumes the volume of the hydrogen system does not change with time. The expression for the volumetric substantial derivative is

$$
-\frac{[H]}{\forall} \frac{d \forall}{d t} \cdot
$$

The magnitude of this term is assumed to be small compared with $R_{d}$ and $R_{r}$ and will therefore be neglected in this analysis.

If the mass fraction of atomic hydrogen $\left(C_{H}\right)$ is defined as the mass of $\mathrm{H}$ divided by the total system mass then the time rate of change of $C_{H}$ for a system can be defined by

$$
\frac{D C_{H}}{D t}=\frac{D[H]}{D t} \frac{M_{H}}{\rho}=\left(R_{d}-R_{r}\right) \frac{M_{H}}{\rho}
$$

where $\rho$ is the system density (which is assumed to be constant) and $M_{H}$ is the molecular weight of $H$.

For a supersonic nozzle operating under quasi-steady conditions the substantial derivative expressing the time rate of change of the mass fraction of $H$ is given by 


$$
\frac{D C_{H}}{D t}=u \frac{\partial C_{H}}{\partial X}
$$

where $u$ is the local velocity and $X$ is the distance downstream from the nozzle entrance.

Consider a supersonic nozzle operating under quasi-steady conditions and assume that equilibrium flow exists up to the nozzle throat. Near the throat the local fluid velocities will be small, the chemical reaction rates will be 1 arge, and the characteristic chemical relaxation times will be small compared to the time that it takes a fluid element to move a fractional distance down the nozzle. Consequently the propellant flowfield will be very close to chemical equilibrium, and for this analysis it will be assumed to be in complete equilibrium. As a fluid element accelerates down the nozzle, the molar densities of the species and the local static temperature will drop and these two effects will affect the flowfield significantly. The effect of dropping the molar densities of the reacting species can be seen by examining Eq. 5, which shows that dropping the molar densities decreases the net reaction rate and this in turn increases the characteristic fluid particle chemical relaxation time. The effect of dropping the local static temperature is brought out by considering the forward and backward rate constants used in Eqs. 3 and 4. The backward rate constants used in Eqs. 3 and 4 are respectively

$$
\begin{aligned}
& K_{b_{H_{2}}}=\frac{7.5 \times 10^{12}}{T} \frac{m^{6}}{K m o l e^{2} \mathrm{sec}} \\
& K_{b_{H}}=\frac{5.9 \times 10^{13}}{T} \frac{m^{6}}{K m o l e^{2} \mathrm{sec}} .
\end{aligned}
$$


For either Eq. 3 or Eq. 4 the forward and backward rate constants are related by the following expression

$$
\frac{K_{f}}{K_{b}}=B\left\{\operatorname { e x p } [ - \frac { D _ { 0 } } { K T } ] \left\{\left\{1-\exp \left[-\frac{\theta_{v}}{T}\right]\right\} T^{\frac{1}{L}}\right.\right.
$$

where

$$
\begin{aligned}
& B=78.01 \frac{\mathrm{Kinole}}{\mathrm{m}^{3} \mathrm{~K}^{\frac{1}{2}}} \\
& \frac{D_{0}}{K}=51960^{\circ} \mathrm{K} \\
& \theta_{v}=6338.3^{\circ} \mathrm{K} .
\end{aligned}
$$

This equation, which is actually a form of the equilibrium constant for the $\mathrm{H}_{2}: 2 \mathrm{H}$ reaction, can be derived by using the results of statistical mechanics and by assuming that electronic excitation is not important and that the $\mathrm{H}_{2}$. molecule behaves as a harmonic oscillator. Examination of Eq. 8 illustrates that as the local static temperature drops, the ratio $K_{f} / K_{b}$ becomes sinall very quickly (this is due to the exponential term involving the chemical energy $D_{0}$ which dominates the expression). inis effectively causes $R_{d}$ to become smaller than $R_{r}$ (hence recombination is favored).

Using these results the Bray sudden freezing criterion can be stater in the following ways

1) Near the throat the flow velocity is not large and the chemical relaxation rates are large because the local temperatures and the reacting specie molar densities are nigh. In this region the characteristic chemical relaxation times are small compared with the time that it takes a fluid

$$
C-2
$$


element to move a fractional distance down the nozzle and consequently the flow is assumed to be in chemical equilibrium.

2) In the flow downstream of the throat, the molar densities and the temperatures drop while the flow velocits increases. The chemical molar densities lag farther and farther behind the equilibrium values and finite rate reactions begin to become important.

3) The propeliant eventually reaches a point where the net rate of chemical reaction is sufficiently small and the local velocity is sufficiently large so that the partial derivative $\partial C_{H} / \partial X$ beconies small. Downstream of this peint in the flowfield the propellant is essentially chemically nonreacting and it is assumed to be fixed in composition (frozen). The characteristic chemical relaxation times are large compared to the time required to tranverse the flowfield.

4) The transition from equilibrium to freezing occurs very quickly within a narrow region, and the location of this region is defined when the following relation is met

$$
u\left|\frac{\partial C_{H}}{\partial X}\right|_{e}=\frac{M_{H}}{v} R_{d} N
$$

where the subscript e denotes equilibrium conditions and $\mathrm{N}$ is a constant of the order of unity. This expression states that the narrow freezing region is located at the point where the local substantial derivative of the mass fraction of $\mathrm{H}$ is of the same order as the dissociation term appearing in Eq. 6. This implies that $R_{r}$ is beginning to become larger 
than $R_{d}$. Consequently, the flow is beginning to depart from chemical equilibrium and the characteristic chenical relaxation times are increasing. A value of unity was used for $N$ in this analysis, which was suggested by Reference $\mathrm{Cl}$.

In the analyses conducted it was found that the supersonic nozzle flowfields were typically near chemical equilibrium conditions. This is due to the fact that the propellant is typically operating under high pressure and high temperature conditions and the nozzle expansion ratios are not large. Because vibrational, rotational and translational modes of energy storage equilibrate more readily than chemical phenomena it is argued that these modes will also be near equilibrium conditions. This assumption has. been applied throughout this analysis. 


\section{APPENDIX D}

UNSTEADY GAS DYNAMICS EFFECTS 


\section{APPENDIX D NOMENCLATURE}

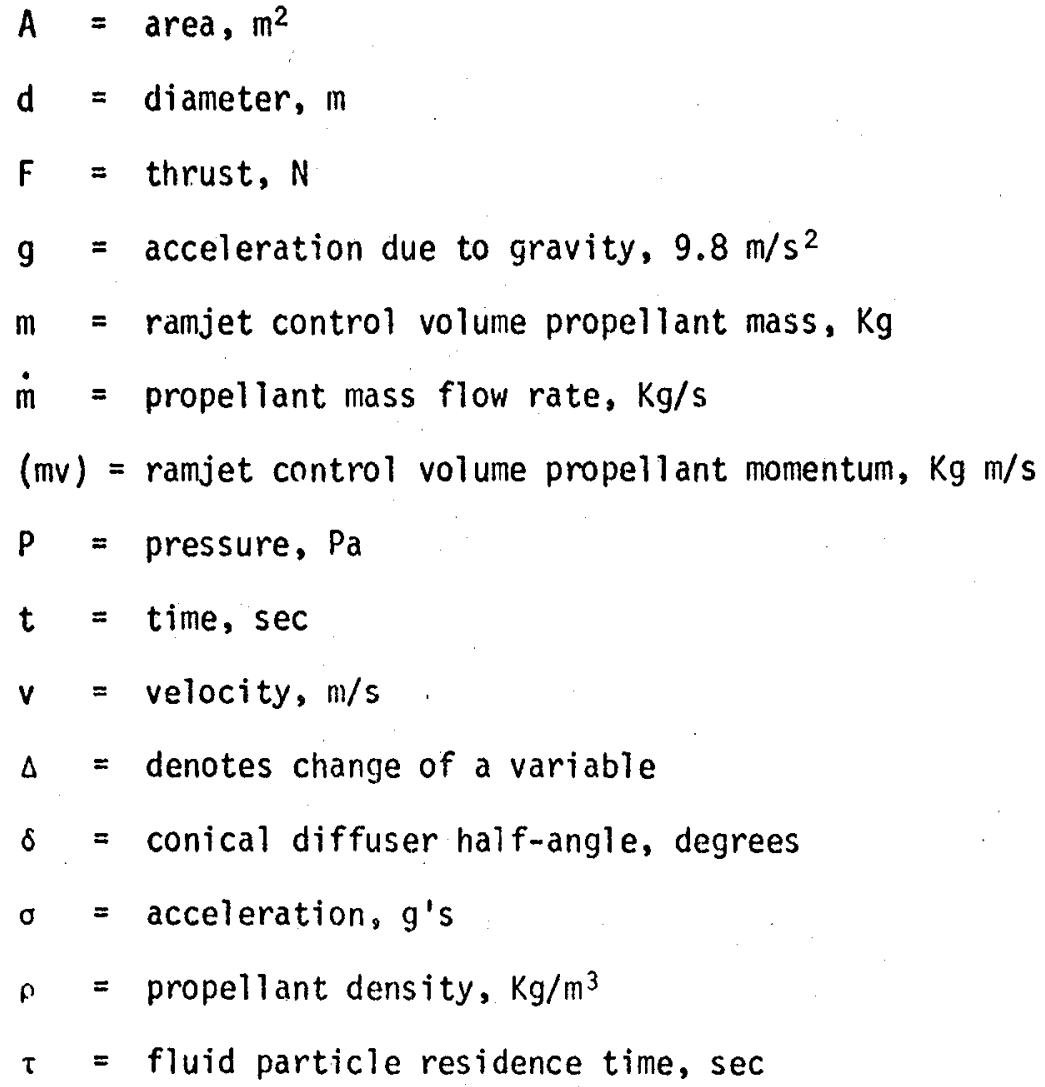

A subscript on a variable denotes conditions at the corresponding location shown in Fig. 01 . 


\section{APPENDIX D \\ UNSTEADY GAS DYNAMICS EFFECTS}

The analyses presented thus far in this thesis have assumed that the propellant flow can be considered to be in a quasi-steady state. In reality, however, the flowfield variables will be changing with respect to time as the electrothermal ramjet accelerates down a launch tube, and it is possible that errors will be introduced by assuming quasi-steady flowfields. It is the purpose of this Appendix to present preliminary calculations which address the effects of unsteadiness.

In order to anaiyze unsteadiness effects it is helptul to consider the residence time $(\tau)$ of a typical fluid particle in the ramjet and to estimate the flowfield changes that will occur over this residence time. Values for $\tau$ will be calculated from the quasi-steady analyses used to generate the results presented earlier in this thesis. Values for fluid particle residence times are generated by specifying a geometric configuration and calculating the time that it takes a fluid particle to pass through this configuration when the internal propellant velocities relative to the projectile are known. The geometric configuration selected (Figure $D I$ ) has a conical half-angle $(\delta)$ of $30^{\circ}$, an annular diffuser throat diameter $\left(d_{3}\right)$ of $16 \mathrm{~cm}$, an annular heat addition rejion diameter $\left\{d_{4}\right)$ of $8 \mathrm{~cm}$, a heat addition region length of $1.0 \mathrm{~m}$, and a total projectile length of $1.25 \mathrm{~m}$. Three separate projectile velocity leveis of $4000 \mathrm{~m} / \mathrm{s}, 7000 \mathrm{~m} / \mathrm{s}$ and $15,000 \mathrm{~m} / \mathrm{s}$ will be examined and one value for the thrust per unit tube area $\left(F / A_{1}\right)$ will be examined for each velocity. For $4000 \mathrm{~m} / \mathrm{s}$ the thrust per unit tube area is $6.7 \times 10^{7} \mathrm{~Pa}$ and for $7000 \mathrm{~m} / \mathrm{s}$ and $15000 \mathrm{~m} / \mathrm{s}$ the values for the thrust per unit tube area are 


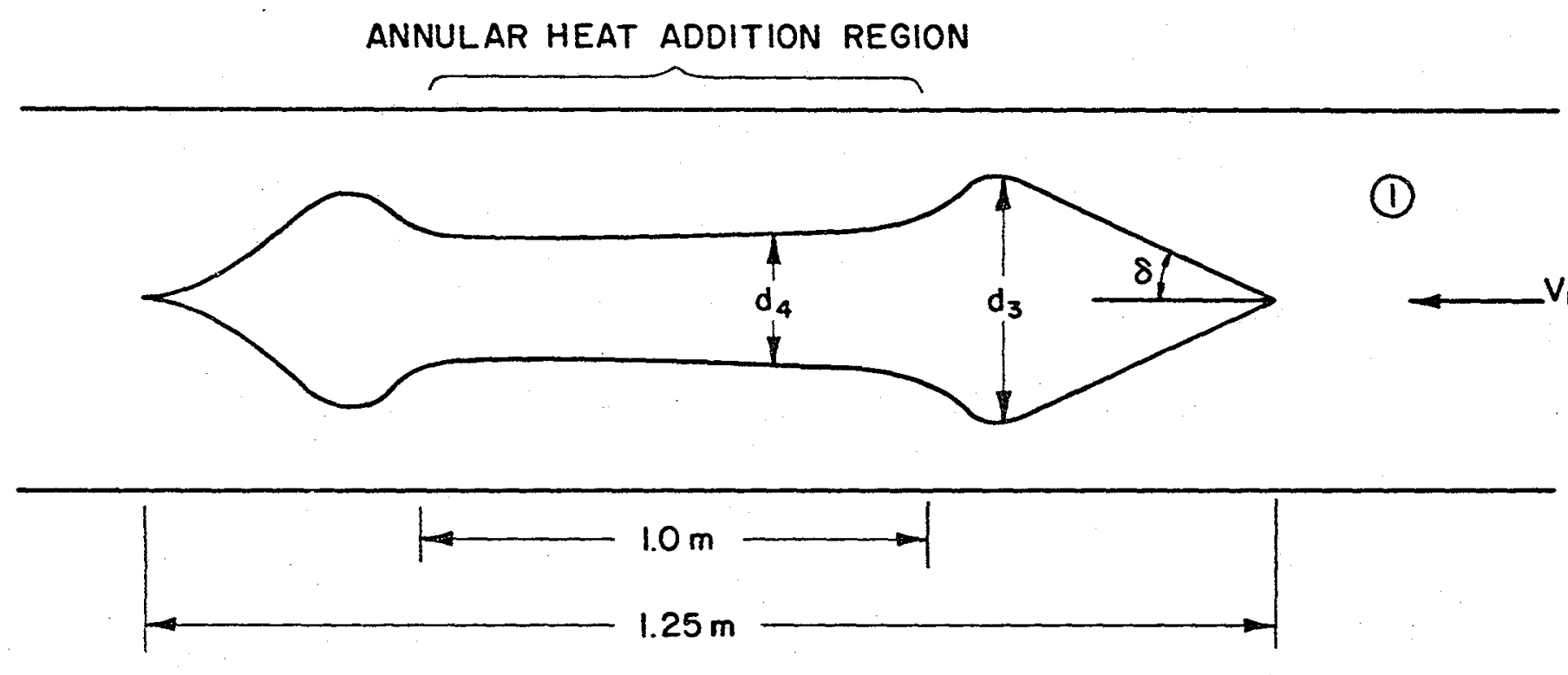

Fig. Di Electrothermal Ramjet Dimensions 
$9.5 \times 10^{7} \mathrm{~Pa}$ and $1.1 \times 10^{8} \mathrm{~Pa}$ respectively. The calculated fluid particle residence times for $4000 \mathrm{~m} / \mathrm{s}, 7000 \mathrm{~m} / \mathrm{s}$ and $15000 \mathrm{~m} / \mathrm{s}$ are respectively

$$
\begin{aligned}
{ }^{\mathrm{t}} 4000 & =0.769 \times 10^{-3} \mathrm{sec} \\
{ }^{\mathrm{T}} 7000 & =1.36 \times 10^{-3} \mathrm{sec} \\
{ }^{\mathrm{t}}{ }_{15000} & =0.861 \times 10^{-3} \mathrm{sec} .
\end{aligned}
$$

The values for the non-dimensional thrust that corresporid to these three velocities are

$$
\begin{aligned}
& \left(\frac{F}{P_{1} A_{1}}\right)_{4000}=6 \\
& \left(\frac{F}{P_{1} A_{1}}\right)_{7000}=10 \\
& \left(\frac{F}{P_{1} A_{1}}\right)_{15000}=70 .
\end{aligned}
$$

It is noted that the fluid particle residence times are primarily dominated by the length of time that a particle spends in the heat addition region of a ramjet, and in order for the residence times to be calculated with high degrees of accuracy it is necessary that the details of the heat addition be known. Also, increasing the amount of heat added per unit mass of propellant increases the average propellant velocity in the heat addition region. This in turn causes the heat addition region residence time to decrease. This explains why there is no definite trend for the residence times presented earlier. The differences are due primarily to the selection of the heat addition rate per unit mass of propellant, and these residence times were selected as being representative of what could occur in an actual device operating with the geometric configuration specified. 
During a residence time $(\tau)$ the ramjet velocity $(v)$ will increase by an amount $\Delta v$. It is instructive to calculate the ratio $\Delta v / v$, which is the ratio of the change in velocity over the time $\tau$ to the velocity of the ramjet at the beginning of the time $\tau$. For constant acceleration this ratio is given by

$$
\frac{\Delta V}{V}=\frac{\Delta g \tau}{V} .
$$

Using an acceleration level (o) of $30,000 \mathrm{~g}$ 's, which will be used throughout this Appendix, slong with the values for the residence times just calculated gives the following normalized changes in projectile velocity

$$
\begin{aligned}
& \left(\frac{\Delta v}{v}\right)_{4000}=0.057 \\
& \left(\frac{\Delta v}{v}\right)_{7000}=0.057 \\
& \left(\frac{\Delta v}{v}\right)_{15000}=0.017 .
\end{aligned}
$$

These values are reasonable and suggest that the projectile velocity will not change significantiy during typical fluid particle residence times for an acceleration level of $30,000 \mathrm{~g}$ 's.

It is also possibie that propellant mass and propellant momentum will accumulate within the ramjet control volume, and it is instructive to consider the control volume flowfield changes over the fluid particle residence times. Consider first the possibility of mass accumulation for a projectile undergoing a constant acceleration. At any time t the influx of mass is given by 


$$
\dot{m}_{\text {in }}=A_{1} \rho_{1} V_{1}
$$

The change in the ramjet control volume mass due to the change in the incoming mass flow as the projectile accelerates during the residence time $\tau$ can be approximated by

$$
\Delta m=\left(\dot{m}_{t+r}-\dot{m}_{t}\right) \tau,
$$

and this can be divided by the mass of propellant in the ramjet (m) to determine the ratio $\Delta \mathrm{m} / \mathrm{m}$ which should be small.

At any time $t$ the momentum flow entering a ramjet control volume is given by

$$
\frac{d}{d t}(m v)=\rho_{1} A_{1} v_{1}^{2}
$$

where (mv) is the ramjet control volume propellant momentum relative to the ramjet, and the amount of momentum that will enter a ramjet control volume over a residence time $t$ can be approximated by

$$
\Delta(m v)=\left(\frac{d}{d t}(m v)_{t+\bar{\tau}} \frac{d}{d t}(m v)_{\tau}\right) \tau .
$$

Again the ratio of this momentum change to the propellant momentum relative to the ramjet. (mv) should be small if unsteady effects are to be negligible. Computation of the ratios $\Delta m / m$ and $\Delta(m v) /(m v)$ have been performed and the results are shown below. For each calculation, it has been assumed that the density of the incoming propellant does not change over a residence time (though increases in velocity would ordinarily imply reductions in density).

$$
\left(\frac{\Delta m}{m}\right)_{4000}=3.8 \times 10^{-2}
$$




$$
\begin{aligned}
& \left(\frac{\Delta m}{m}\right)_{7000}=5.57 \times 10^{-2} \\
& \left(\frac{\Delta m}{m}\right)_{15000}=1.32 \times 10^{-6} \\
& \left(\frac{\Delta(m v)}{(m v)}\right)_{4000}=0.11 \\
& \left(\frac{\Delta(m v)}{(m v)}\right)_{7000}=0.45 \\
& \left(\frac{\Delta(m v)}{(m v)}\right)_{15000}=0.14
\end{aligned}
$$

The above resuits suggest the errors associated with the changes in mass are not large, while the errors associated with the changes in propellant control volume momentum are more significant. It is noted, however, that proper control of the propellant density immediately upstream of the accelerating ramjet will allow these momentum errors to be reduced. The errors that were estimated are felt to be at the upper limit of what might actually occur and although a complete analys is has not been carried out, intuitively it is felt that the ramjet operational conditions could be controlled in a way that would make these errors insignificant. For example, it is apparent that the propellant density should decrease as ramjet velocity increases so that mass and momentum accumulation will be minimized, and this decrease in density is also required for optimum quasi-steady operation (see Figure 12).

Another area of concern is associated with the fact that the propellant in the heat addition region is being accelerated, and it is possible that a "hydrostatic" pressure gradient could be produced in 
the heat addition region. This problem was also analyzed for the cases presented earlier and results for the ratio $\triangle P / P$ were computed. In this ratio $\Delta P$ is the "hydrostatic" pressure difference across the heat addition region and $P$ is the average quasi-steady pressure calculated for the heat addition region. Computed values of this ratio are

$$
\begin{aligned}
& \left(\frac{\Delta P}{P}\right)_{4000}=0.064 \\
& \left(\frac{\Delta P}{P}\right)_{7000}=0.028 \\
& \left(\frac{\Delta P}{P}\right)_{15000}=0.0075 .
\end{aligned}
$$

These values are considered not large enough to introduce significant errors intu the results presented thus far.

In conclusion, it is felt that the errors incurred by assuming quasi-steady operation, while significant, are not sufficiently large to suggest the results presented are grossly in error. Further it is suggested that these errors can be reduced significantly by proper 1 aunch trajectory design. It is also noted that these results were obtained for a specific geometric configuration and acceleration level. Decreasing the various dimensions presented earlier and decreasing the acceleration level will decrease the errors associated with unsteady gas dynamics effects. 
End of Document 